\title{
Superspace conformal field theory
}

\author{
Thomas Quella ${ }^{1}$ and Volker Schomerus ${ }^{2}$ \\ ${ }^{1}$ Universität zu Köln, Institut für Theoretische Physik, Zülpicher Straße 77, 50937 Cologne, Germany \\ ${ }^{2}$ DESY, Theory Group, Notkestrasse 85, Bldg. 2a, 22603 Hamburg, Germany \\ Emails: Thomas.Quella@uni-Koeln.de, Volker.Schomerus@desy.de
}

\begin{abstract}
Conformal sigma models and WZW models on coset superspaces provide important examples of logarithmic conformal field theories. They possess many applications to problems in string and condensed matter theory. We review recent results and developments, including the general construction of WZW models on type I supergroups, the classification of conformal sigma models and their embedding into string theory.
\end{abstract}

Keywords: Review, Logarithmic Conformal Field Theory, Conformal Field Theory, String Theory, Supersymmetry, Lie Superalgebras, Representation Theory, Supergeometry, Harmonic Analysis.

\section{Contents}

1 Introduction 2

2 Lie superalgebras 4

2.1 Definition and examples of Lie superalgebras . . . . . . . . . . . . . 4

2.2 Classification of Lie superalgebras . . . . . . . . . . . . . . . . . 6

2.3 Representation theory ........................ 7

2.3.1 Introduction and overview ...................... 7

2.3.2 Category theoretic perspective .................. 11

2.4 Harmonic analysis on supermanifolds . . . . . . . . . . . . . . . . . 12

2.4.1 Guide to the literature on supergeometry . . . . . . . . . . 12

2.4 Supergroups......................... 13

2.4 .3 Harmonic analysis on supercosets . . . . . . . . . . . . 16

2.5 Cohomological reduction . . . . . . . . . . . . . . . . . 17

2.5 .1 General formalism ......................... 17

2.5.2 Application: Function spaces on supercosets . . . . . . . . . 18

3 Supergroup WZW models $\quad 19$

3.1 The action functional . . . . . . . . . . . . . . . . . . . 19

3.2 Free fermion realization for type I supergroups . . . . . . . . . . . . . . . . . 20

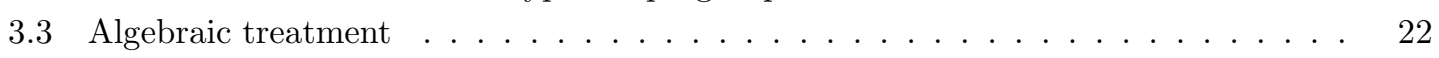

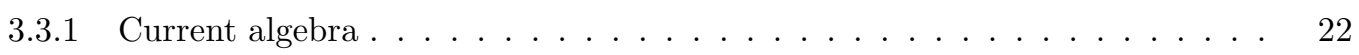

3.3.2 Free fermion realization . . . . . . . . . . . . . . . 23

3.3.3 Chiral representation theory ................... 24

3.3.4 Spectral flow automorphisms .................. 25

3.3.5 The partition function: Decoupled versus interacting theory . . . . . . 26 
3.4 Free field realization the $\operatorname{OSP}(m \mid 2 n)$ WZW model at level $k=1 \ldots \ldots$

3.4 Current algebra . . . . . . . . . . . . . . 28

3.4 .2 Comments on the state space . . . . . . . . . . . . 29 29

3.5 Deformations .................................. 31

3.5.1 $\mathrm{G} \times \mathrm{G}$ preserving deformations ......................... 31

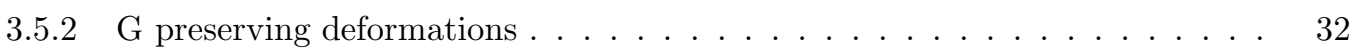

3.5.3 Exact marginality for quasi-abelian supergroups . . . . . . . . . 32

3.5.4 Anomalous dimensions ...................... 33

4 Superspace sigma models and strings 34

4.1 Sigma models on coset superspaces . . . . . . . . . . . . . . . . . . 34

4.1 Basic formulation . . . . . . . . . . . . . . . 34

4.1.2 Symmetric superspaces and conformal invariance . . . . . . . . . . . . 35

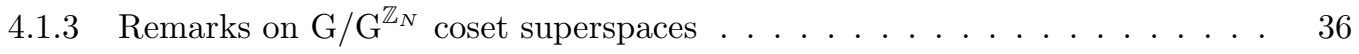

4.2 Embedding into string theory . . . . . . . . . . . . . . . . 37

4.2.1 The coset space $\operatorname{PSU}(1,1 \mid 2) / \mathrm{U}(1) \times \mathrm{U}(1) \ldots \ldots \ldots$

4.2 .2 String theory on $\mathrm{AdS}_{2} \times \mathrm{S}^{2} \times \mathrm{CY}_{6} \ldots \ldots \ldots \ldots$

4.2 .3 Other string backgrounds - an overview . . . . . . . . . . . . 39

4.3 A duality between Gross-Neveu and supersphere sigma models . . . . . . . . . . 40

4.3.1 The $\operatorname{OSP}(2 S+2 \mid 2 S)$ Gross-Neveu model . . . . . . . . . . . . . . 40

4.3.2 Deformed boundary spectrum . . . . . . . . . . . . . . 41

4.3.3 Identification with supersphere sigma model . . . . . . . . . 42

5 Conclusions and Outlook 43

\section{Introduction}

Two-dimensional conformal field theories with central charge $c=0$, or other values below the unitarity bound $c=1 / 2$, play a fundamental role for string theory as well as statistical mechanics. With a few isolated exceptions, most models within this class possess world-sheet correlation functions with logarithmic divergencies. With the lack of chiral factorization and other familiar features of 2D CFT, such non-unitary models provide a major challenge for modern mathematical physics. In this context, internal supersymmetry, i.e. the existence of supersymmetry transformations that act in field space while leaving world-sheet positions invariant, can help to make models more tractable. Our aim here is to review recent progress in conformal field theory that is linked to internal supersymmetry. Almost all models to be discussed below are logarithmic.

Conformal field theories with internal supersymmetries have been a topic of considerable interest for the past few decades. Their realm of applications is vast, ranging from string theory to statistical physics and condensed matter theory. In the Green-Schwarz or pure spinor type formulation of superstring theory, for example, supersymmetries act geometrically as isometries of an underlying space-time (target space) supermanifold. Important examples arise in the context of AdS/CFT dualities between supersymmetric gauge theories and closed strings [1. Apart from string theory, supersymmetry has also played a major role in the context of quantum disordered systems [2, 3, 4, and in models with non-local degrees of freedom such as polymers [5, 6]. In particular, it seems to be a crucial ingredient in the description of the plateaux transitions in the spin [7, 8] and the integer quantum Hall effect [9, 10, 11, 12. It is well known for instance that observables in the Chalker-Coddington network model for the integer quantum Hall effect 13 may be expressed as correlation functions in a (non-conformal) non-linear $\sigma$-model on $\mathrm{U}(1,1 \mid 2) / \mathrm{U}(1 \mid 1) \times \mathrm{U}(1 \mid 1)$, 9, 10. However, so far none of the attempts [10, 11, 12, to identify the conformal field theory describing the strong coupling fixed point led to a completely satisfactory picture.

In addition to having such concrete applications, conformal field theories with target space (internal) supersymmetry can teach us important lessons about logarithmic conformal field theory. As 
we indicated above, most models that possess internal supersymmetry exhibit the usual features of non-unitary conformal field theory such as the occurrence of reducible but indecomposable1 representations and the existence of logarithmic singularities on the world-sheet. In this context, many conceptual issues remain to be solved, both on the physical and on the mathematical side. These include, in particular, the construction of consistent local correlation functions [14, the modular transformation properties of characters [15, their relation to fusion rules [16], the treatment of conformal boundary conditions [17] etc. As we shall see below, superspace models provide a wide zoo of theories in which such issues can be addressed with an interesting mix of algebraic and geometric techniques.

In addition, the special properties of Lie supergroups allow for constructions which are not possible for ordinary groups. For instance, there exist several families of coset conformal field theories that are obtained by gauging a one-sided action of some subgroup rather than the usual adjoint [18, 19, 20, 21. The same class of supergroup $\sigma$-models is also known to admit a new kind of marginal deformations that are not of current-current type [22, 23, 24]. Finally, there seems to be a striking correspondence between the integrability of these models and their conformal invariance [25, 26, 20, 21].

Here we shall review recent developments in this direction along with all the required background, in particular from the representation theory of Lie superalgebras. The latter will be discussed in the next section. Special emphasis is put on atypical representations with nondiagonalizable quadratic Casimir because of their direct link with logarithms in the conformal field theory models to be discussed in Section 3. There we shall provide a comprehensive discussion of Wess-Zumino-Witten (WZW) models on target supergroups of type I. These models possess all the features mentioned above. The two essential properties which facilitate an exact solution are i) the presence of an extended chiral symmetry based on an infinite dimensional current superalgebra $2^{2}$ and ii) the inherent geometric interpretation. While ii) is common to all $\sigma$-models, the symmetries of WZW models are necessary to lift geometric insights to the full field theory. Both aspects single out supergroup WZW theories among most of the logarithmic conformal field theories that have been considered in the past [27, 14, 28] (see also [29, 30] for reviews and further references). While investigations of algebraic and mostly chiral aspects of supergroup WZW models reach back more than twenty years [31, 32, 33, 34, 35 the relevance of geometric methods for our understanding of non-chiral issues was realized much later in [36, 24, 37, 38.

WZW models on some special supergroups possess marginal symmetry preserving currentcurrent deformations. These are discussed briefly at the end of Section 3 . In Section 4 we turn to $\sigma$-models on coset superspaces. We begin by spelling out the action and then review what is known about the beta function of these models. The results are most complete for $\sigma$-models on symmetric superspaces. Some of these $\sigma$-models are used as building blocks of exact string backgrounds for strings moving in Anti de-Sitter spaces. We shall only outline one example along with a short guide to the existing literature. The last subsection illustrates how deformed WZW models and $\sigma$-models can appear as alternative descriptions of one and the same theory. In order to do so, we start with a WZW model for the supergroup $\operatorname{OSP}(2 S+2 \mid 2 S)$ and deform it through a current-current interaction. Following [39] we shall argue that, for large coupling constant, the model is driven to a weakly curved $\sigma$-model on the superspace $\mathrm{S}^{2 S+1 \mid 2 S}$. This does not only provide a beautiful link between different parts of this review but could also become a paradigm for more general dualities between WZW and conformal $\sigma$-models.

As a final step before diving into the main subject we wish to provide a brief outline of how this review fits into the Special Issue on Logarithmic Conformal Field Theory it belongs to. The closest relationship exists to the review by Gainutdinov, Jacobsen, Saleur and Vasseur on "Lattice Regularizations of Logarithmic Conformal Field Theories", which - among others - also discusses supersymmetric spin systems. Some of our theories can be understood as the continuum description of the IR fixed points of their lattice models. Besides, the importance of the omni-present $c=$

\footnotetext{
${ }^{1}$ In contrast to some appearances in the physics literature we will use the word "indecomposable" strictly in the mathematical sense. According to that definition also irreducible representations are always indecomposable since they cannot be written as a direct sum of two other (non-zero) representations.

2Instead of referring to the names "Kac-Moody superalgebra" or even "affine Lie superalgebra" which are frequently used in the physics community, we will stick to the notion current superalgebra by which we mean a central extension of the loop algebra over a finite dimensional Lie superalgebra.
} 


\begin{tabular}{cccccc} 
Name & Alternative name & $\underline{g}_{\underline{0}}$ & $\mathfrak{g}_{\underline{1}}$ & $g^{\vee}$ & Defect \\
\hline \hline$A(m, n)$ & $\operatorname{sl}(m+1 \mid n+1)$ & $A_{m} \oplus A_{n} \oplus T_{1}$ & $\left(\omega_{1}, \omega_{n}\right)_{+} \oplus\left(\omega_{m}, \omega_{1}\right)_{-}$ & $|m-n|$ & $\min (m, n)$ \\
$A(n, n)$ & $\operatorname{psl}(n \mid n)$ & $A_{n} \oplus A_{n}$ & $\left(\omega_{1}, \omega_{n}\right) \oplus\left(\omega_{n}, \omega_{1}\right)$ & 0 & $n$ \\
$C(n)$ & $\operatorname{osp}(2 \mid 2 n-2)$ & $C_{n-1} \oplus T_{1}$ & $\left(\omega_{1}\right)_{+} \oplus\left(\omega_{1}\right)_{-}$ & $n-1$ & 1 \\
\hline$F(4)$ & - & $A_{1} \oplus B_{3}$ & $\left(\omega_{1}, \omega_{3}\right)$ & 3 & 1 \\
$G(3)$ & - & $A_{1} \oplus G_{2}$ & $\left(\omega_{1}, \omega_{2}\right)$ & 2 & 1 \\
$B(m, n)$ & $\operatorname{osp}(2 m+1 \mid 2 n)$ & $B_{m} \oplus C_{n}$ & $\left(\omega_{1}, \omega_{1}\right)$ & $f_{m, n}$ & $\min (m, n)$ \\
$D(m, n)$ & $\operatorname{osp}(2 m \mid 2 n)$ & $D_{m} \oplus C_{n}$ & $\left(\omega_{1}, \omega_{1}\right)$ & $g_{m, n}$ & $\min (m, n)$ \\
$D(2,1 ; \alpha)$ & - & $A_{1} \oplus A_{1} \oplus A_{1}$ & $\left(\omega_{1}, \omega_{1}, \omega_{1}\right)$ & 0 & 1 \\
\hline
\end{tabular}

Table 1: Classical Lie superalgebras with non-degenerate even invariant form, sorted according to whether they are of type I (top) or type II (bottom). The symbol $T_{1}$ denotes the abelian Lie algebra $\mathrm{gl}(1)$ of dimension one, $g^{\vee}$ is the dual Coxeter number, see text after eq. (2.6), and the definition of 'defect' is given in the text following eq. (2.19). The $\omega_{i}$ are fundamental weights in the conventions of [42, while the subscripts \pm refer to two mutually dual non-trivial representations of $T_{1}$. Furthermore one has $f_{m, n}=2(m-n)-1$ and $g_{m, n}=2(m-n-1)$ for $m>n$ and $f_{m, n}=n-m+1 / 2$ and $g_{m, n}=n-m+1$ for $m \leq n$ (for the data see [46]).

-2 ghost system in our treatise provides a natural link to many of the other contributions, in particular to Creutzig and Ridout's review on "Logarithmic Conformal Field Theory: Beyond an Introduction". We should also note that, from a physical perspective, our review is somewhat complementary to Cardy's in the sense that disordered systems can also be described using the supersymmetry trick instead of the replica method [3, 4]. However, this is an aspect we do not emphasize here. Finally, it is an outstanding problem how the results discussed in our review can be embedded into the rigorous mathematical setting described by Adamovic and Milas on the one hand and Huang and Lepowsky on the other.

\section{Lie superalgebras}

The theory of Lie superalgebras was developed by Kac 40 and, independently, by Nahm, Scheunert and Rittenberg [41. Quite a few notions from ordinary Lie theory carry over with only minor changes. On the other hand, there are also substantial differences, mostly related to the existence of reducible but indecomposable representations and to the possibility of choosing inequivalent systems of simple roots. With this section we lay the mathematical foundations which will enable us to carve out the peculiarities of superspace conformal field theories in subsequent sections.

We assume that the reader has some familiarity with the structure and the representation theory of ordinary Lie algebras. If this is not the case, we recommend to consult one of the references 42, 43, 44. A comprehensive overview, covering most of the more standard aspects of Lie superalgebras is the "Dictionary on Lie superalgebras" 45.

\subsection{Definition and examples of Lie superalgebras}

The notion of a Lie superalgebra is a relatively straightforward generalization of that of a Lie algebra, taking into account the possibility of having fermionic (or odd) generators. As a consequence, an arbitrary Lie superalgebra $\mathfrak{g}$ splits into an even part $\mathfrak{g}_{\underline{0}}$ and an odd part $\mathfrak{g}_{1}$. A Lie superalgebra is thus a $\mathbb{Z}_{2}$-graded vector space $\mathfrak{g}=\mathfrak{g}_{\underline{0}} \oplus \mathfrak{g}_{\underline{1}}$ with a bilinear Lie bracket $[\cdot, \cdot]: \mathfrak{g} \otimes \mathfrak{g} \rightarrow \mathfrak{g}$ which satisfies some natural graded modifications of the axioms for a Lie algebra. First of all, the bracket is consistent with the grading in the sense that $\left[\mathfrak{g}_{\underline{i}}, \mathfrak{g}_{j}\right] \subset \mathfrak{g}_{i+j}$. This condition ensures that $\mathfrak{g}_{\underline{1}}$ can be regarded as a representation of $\mathfrak{g}_{\underline{0}}$. We shall later see that the precise nature of $\mathfrak{g}_{\underline{1}}$ as a $\mathfrak{g}_{0}$-module determines many of the most important properties of $\mathfrak{g}$. Secondly, the bracket is graded anti-symmetric, i.e. $[X, Y]=-(-1)^{d_{X} d_{Y}}[Y, X]$ for all homogeneous elements $X, Y \in \mathfrak{g}$ and with $d_{\bullet}: \mathfrak{g}_{\underline{0}} \cup \mathfrak{g}_{\underline{1}} \rightarrow \mathbb{Z}_{2}$ denoting the grade function. And last but not least, for homogeneous elements 
$X, Y, Z \in \mathfrak{g}$ one has the graded Jacobi identity

$$
[X,[Y, Z]]+(-1)^{d_{Z}\left(d_{X}+d_{Y}\right)}[Z,[X, Y]]+(-1)^{d_{X}\left(d_{Y}+d_{Z}\right)}[Y,[Z, X]]=0 .
$$

The Jacobi identity may be viewed as a constraint which ensures the existence of an adjoint representation, the representation of $\mathfrak{g}$ on itself (see below). In our paper, we will only be interested in Lie superalgebras over the field of complex numbers.

In most, if not all, physical applications it is essential to have a metric to measure lengths and distances. In the context of Lie superalgebras, the relevant notion is that of a non-degenerate symmetric even invariant bilinear form $\langle\cdot, \cdot\rangle: \mathfrak{g} \otimes \mathfrak{g} \rightarrow \mathbb{C}$. Invariance refers to the condition

$$
\langle[X, Y], Z\rangle=\langle X,[Y, Z]\rangle,
$$

while symmetry requires $\langle X, Y\rangle=(-1)^{d_{X} d_{Y}}\langle Y, X\rangle$. The form is even if $\left\langle\mathfrak{g}_{\underline{i}}, \mathfrak{g}_{\underline{j}}\right\rangle=0$ whenever we pair the even and the odd part $(\underline{i} \neq \underline{j})$.

Let us now discuss a few standard examples of Lie superalgebras. For this purpose we consider a complex vector superspace $V=V_{\underline{0}} \oplus V_{\underline{1}}$ of dimension $m \mid n$. In other words, $m=\operatorname{dim}\left(V_{\underline{0}}\right)$ and $n=\operatorname{dim}\left(V_{\underline{1}}\right)$ and the combinations $m+n$ and $m-n$ are known as the dimension and the superdimension of $V$, respectively. The space of linear maps $\operatorname{End}(V)$ naturally inherits the structure of a vector superspace (actually of an associative superalgebra) by using the assignment

$$
\begin{aligned}
& \operatorname{End}(V)_{\underline{0}} \stackrel{\text { def }}{=} \operatorname{Hom}\left(V_{\underline{0}}, V_{\underline{0}}\right) \oplus \operatorname{Hom}\left(V_{\underline{1}}, V_{\underline{1}}\right) \quad \text { and } \\
& \operatorname{End}(V)_{\underline{1}} \stackrel{\text { def }}{=} \operatorname{Hom}\left(V_{\underline{0}}, V_{\underline{1}}\right) \oplus \operatorname{Hom}\left(V_{\underline{1}}, V_{\underline{0}}\right) \text {. }
\end{aligned}
$$

In fact, $\operatorname{End}(V)$ can easily be seen to give rise to a Lie superalgebra by identifying the Lie bracket with the (anti-)commutator

$$
[X, Y] \stackrel{\text { def }}{=} X Y-(-1)^{d_{X} d_{Y}} Y X
$$

It is a straightforward exercise to verify all relevant axioms of a Lie superalgebra. Frequently, the Lie superalgebra above is also referred to as $\operatorname{gl}(V)$ or $\operatorname{gl}(m \mid n)$. It is called the general linear superalgebra.

The elements of $\mathrm{gl}(m \mid n)$ can be conveniently expressed as matrices. If one uses an adapted basis with the first part of basis elements from $V_{\underline{0}}$ and the remaining ones from $V_{\underline{1}}$, the matrices $M \in \operatorname{gl}(m \mid n)$ will have the block form $M=\left(\begin{array}{cc}A & \bar{B} \\ C\end{array}\right)$ with $A, D$ being even and $B, C$ being odd 3 In this basis, the supertrace is defined by $\operatorname{str}\left(\begin{array}{c}A \\ C\end{array} \stackrel{D}{D}\right)=\operatorname{tr}(A)-\operatorname{tr}(D)$. As a slight variation of $\operatorname{gl}(m \mid n)$ we can consider the subspace $\operatorname{sl}(m \mid n)$ of all matrices with vanishing supertrace. It can be checked that this subspace is closed under the Lie bracket (2.5) and that it hence gives rise to a Lie superalgebra as well, also known as the special linear superalgebra. As it turns out, the case of $\operatorname{sl}(n \mid n)$ is rather special. While $\operatorname{sl}(m \mid n)$ is simple for $m \neq n$, i.e. there are no non-trivial ideals, the Lie superalgebra $\operatorname{sl}(n \mid n)$ has an abelian ideal $\operatorname{gl}(1)$ which is generated by multiples of the identity matrix. The quotient $\operatorname{psl}(n \mid n)=\operatorname{sl}(n \mid n) / \operatorname{gl}(1)$ is simple. Due to its peculiar properties it will accompany us throughout most of this review.

One more important family of Lie superalgebras, the orthosymplectic superalgebras $\operatorname{osp}(m \mid 2 n)$, can be introduced as a subspace of $\mathrm{gl}(m \mid 2 n)$ if one demands that the maps leave invariant a quadratic form which is orthogonal on $\mathbb{C}^{m \mid 0}$ and symplectic on $\mathbb{C}^{0 \mid 2 n}$. Such a form is typically chosen to be represented by the block matrix $\left(\begin{array}{ccc}\text { id } & 0 & 0 \\ 0 & 0 & \text { id } \\ 0 & - \text { id } & 0\end{array}\right)$.

One of the most important concepts in the theory of Lie superalgebras are Casimir operators, in particular quadratic Casimir operators. Given any metric $\langle\cdot, \cdot\rangle$ one can define the graded symmetric invariant tensor $\kappa^{a b}=\left\langle T^{a}, T^{b}\right\rangle$ with respect to some homogeneous basis. The associated quadratic Casimir operator is then defined by the equation

$$
C=\kappa_{b a} T^{a} T^{b}
$$

\footnotetext{
${ }^{3}$ In order to avoid confusion we should stress that the entries of all matrices are complex numbers. There are no Grassmann variables involved here.
} 
where $\kappa_{a b}$ denotes the inverse of the matrix $\kappa^{a b}$. For simple Lie superalgebras, the quadratic Casimir operator is unique up to normalization. In the standard normalization where long roots have length 2, half the value of the quadratic Casimir operator in the adjoint representation is known as the dual Coxeter number $g^{\vee}$ of the Lie superalgebra $\mathfrak{g}$ (see 42 ).

Setting $\operatorname{ad}_{X}=[X, \cdot] \in \operatorname{End}(\mathfrak{g})$, let us finally recall the notion of the Killing form

$$
K(X, Y)=\operatorname{str}\left(\operatorname{ad}_{X} \circ \operatorname{ad}_{Y}\right)
$$

The Killing form plays an essential role in the structure theory of ordinary Lie algebras since semisimple Lie algebras can be characterized by the property that the Killing form is non-degenerate. In contrast, even for simple Lie superalgebras the Killing form may vanish identically. This is the case for the series $\operatorname{psl}(n \mid n)$ and $\operatorname{osp}(2 n+2 \mid 2 n)$. Surprisingly, as we shall see, these Lie superalgebras are in a sense the physically most interesting ones.

Example: The Lie superalgebra $g l(1 \mid 1)$. The Lie superalgebra $g l(1 \mid 1)$ is generated by two even generators $E, N$ and two fermionic generators $\psi^{ \pm}$. While $E$ is central, the generator $N$ can be interpreted as a fermion counting operator. The non-trivial Lie brackets read

$$
\left[N, \psi^{ \pm}\right]= \pm \psi^{ \pm} \quad \text { and } \quad\left[\psi^{+}, \psi^{-}\right]=E
$$

In the fundamental representation, the generators can be represented by

$$
E=\left(\begin{array}{ll}
1 & 0 \\
0 & 1
\end{array}\right), \quad N=\frac{1}{2}\left(\begin{array}{cc}
1 & 0 \\
0 & -1
\end{array}\right), \quad \psi^{+}=\left(\begin{array}{ll}
0 & 1 \\
0 & 0
\end{array}\right), \quad \psi^{-}=\left(\begin{array}{ll}
0 & 0 \\
1 & 0
\end{array}\right)
$$

One can easily check that the most general metric is specified by

$$
\langle E, N\rangle=a,\left\langle\psi^{+}, \psi^{-}\right\rangle=a,\langle N, N\rangle=b
$$

with arbitrary constants $a \neq 0$ and $b$. In contrast, the Killing form is degenerate and easily seen to give rise to $K(N, N)=-2$, with all other components being trivial. The most general quadratic Casimir of gl(1|1) can be expressed as an arbitrary linear combination of the two elements

$$
C=2 E N-\psi^{+} \psi^{-}+\psi^{-} \psi^{+} \quad \text { and } \quad \tilde{C}=E^{2} .
$$

\subsection{Classification of Lie superalgebras}

Simple Lie superalgebras have been classified by Kac in 40. Just as in the case of ordinary Lie algebras, there are several infinite families and a few exceptional cases 4 In all what follows, we shall limit ourselves to classical Lie superalgebras, i.e. simple Lie superalgebras whose odd part $\mathfrak{g}_{1}$ is completely reducible under the action of $\mathfrak{g}_{\underline{0}}$. This excludes the Cartan type Lie superalgebras $W(n), S(n), \tilde{S}(n)$ and $H(n)$. The classical Lie superalgebras furthermore split into the basic ones, i.e. those admitting a metric in the sense of Section 2.1] and the two strange families $P(n)$ and $Q(n)$ for which such a metric does not exist. For physical reasons, we will restrict our attention to the basic Lie superalgebras whose complete list can be found in Table 1 together with a few of their properties. For a detailed description of all simple Lie superalgebras we refer the reader to [40, 45. Apart from the series introduced in Section 2.1 we only wish to highlight the family of exceptional Lie superalgebras $D(2,1 ; \alpha)$ which can be regarded as a deformation of osp $(4 \mid 2)$.

It may be shown that the even part $\mathfrak{g}_{\underline{0}}$ of a classical Lie superalgebra $\mathfrak{g}$ is reductive. In other words, it decomposes into a direct sum of simple or abelian Lie algebras. One then distinguishes furthermore Lie superalgebras of type I and type II. In the type II case, $\mathfrak{g}_{1}$ is irreducible under the action of $\mathfrak{g}_{0}$ while it splits into two irreducibles if $\mathfrak{g}$ is of type I. We shall see below that the properties of Lie superalgebras of type I and II, respectively, are significantly different.

Certain physical applications require to work with non-semisimple Lie superalgebras. Apart from the previously discussed examples $\operatorname{gl}(m \mid n)$ or $\operatorname{sl}(n \mid n)$ this mainly concerns different Poincaré

\footnotetext{
${ }^{4}$ We adopt the convention that when using the term Lie superalgebra we always mean a Lie superalgebra with non-trivial odd part $\mathfrak{g}_{\underline{1}}$. The other Lie superalgebras are referred to as ordinary Lie algebras.
} 


\begin{tabular}{cl} 
Symbol & Meaning \\
\hline \hline $\mathbb{C}_{\lambda}$ & One-dimensional module of the Cartan subalgebra $\mathfrak{h}$ \\
\hline$L_{\lambda}$ & Simple module of $\mathfrak{g}_{0}$ \\
$V_{\lambda}$ & Verma module (induced from $\left.\mathbb{C}_{\lambda}\right)$ \\
\hline $\mathcal{L}_{\lambda}$ & Simple module of $\mathfrak{g}$ \\
$\mathcal{K}_{\lambda}$ & Kac module $\left(\right.$ covering $\left.\mathcal{L}_{\lambda}\right)$ \\
$\mathcal{P}_{\lambda}$ & Projective cover of $\mathcal{L}_{\lambda}$ \\
$\mathcal{B}_{\lambda}$ & Projective module (induced from $L_{\lambda}$ ) \\
$\mathcal{V}_{\lambda}$ & Verma module (induced from $\mathbb{C}_{\lambda}$ ) \\
\hline
\end{tabular}

Table 2: Different types of representations featuring in this article.

superalgebras in various dimensions [4] as they occur in supersymmetric quantum field theories or string theories. In particular, we suspect that many of the problems in the covariant quantization of superstrings are actually due to the lack of a non-degenerate invariant form for the Poincaré superalgebras. For this reason, certain aspects of strings on AdS-spaces (with an underlying simple isometry supergroup and hence the existence of a metric) might actually be easier to deal with than the corresponding ones on flat space, see e.g. [48.

\subsection{Representation theory}

\subsubsection{Introduction and overview}

A representation of $\mathfrak{g}$ on a vector superspace $\mathcal{M}$ is, by definition, a Lie superalgebra homomorphism from $\mathfrak{g}$ to $\mathrm{gl}(\mathcal{M})$. If the relevant homomorphism is clear from the context we will simply refer to $\mathcal{M}$ when describing the representation. We should also mention that we will use the notions representation and module interchangeably. Elementary examples of representations are the trivial representation $\mathcal{L}_{0}: \mathfrak{g} \rightarrow \mathbb{C}$ with kernel $\mathfrak{g}$ and the adjoint representation ad $: \mathfrak{g} \rightarrow \mathfrak{g}$ in terms of the homomorphism $X \mapsto[X, \cdot]$. We reserve the symbol $\mathcal{L}_{\lambda}$ to denote irreducible representations of $\mathfrak{g}$ while $L_{\lambda}$ refers to irreducible representations of the even subalgebra $\mathfrak{g}_{\underline{0}}$. In what follows we shall exclusively be interested in weight modules. These are modules on which the elements of the Cartan subalgebra $\mathfrak{h} \subset \mathfrak{g}$ act diagonally. In the cases of interest, the Cartan subalgebra of $\mathfrak{g}$ may be identified with the Cartan subalgebra of its bosonic subalgebra $\mathfrak{g}_{\underline{0}}$.

There are many invariants which help to characterize representations. However, for the purposes of our paper it will mostly be sufficient to work with the following two: The quadratic Casimir and characters. Since the quadratic Casimir operator (2.6) is an even element which commutes with all generators of $\mathfrak{g}$, it will be represented as a multiple of the identity matrix in any irreducible representation $\mathcal{L}_{\lambda}$. This follows from a superalgebra variant of Schur's Lemma 40. The corresponding constant of proportionality will be denoted by $C(\lambda)$. Representations with different values of $C(\lambda)$ are certainly not isomorphic while, a priori, not much can be said if the values agree.

Just as for ordinary Lie algebras, one can define the direct sum and the tensor product of two representations. For the tensor product one needs to take into account that one is working with graded structures which lead to natural sign factors appearing every now and then. One of the main differences between simple Lie algebras and Lie superalgebras is the occurrence of indecomposable representations which are not irreducible. This leads to a veritable zoo of representations, the most important of which are listed in Table 2. Eventually, the existence of representations which are not fully reducible may be traced back to the nil-potency of certain odd generators. More details and examples will be provided below. The only series of classical Lie superalgebras for which all finite dimensional representations are fully reducible is $B(0, n)=\operatorname{osp}(1 \mid 2 n)$.

The natural method of constructing non-trivial representations for a Lie superalgebra $\mathfrak{g}$ is using induction from a subalgebra $\mathfrak{k}$. Here one starts from a representation $\mathcal{M}_{\mathfrak{k}}$ of $\mathfrak{k}$ and uses the known commutation relations between the generators of $\mathfrak{k}$ and $\mathfrak{g}$ in order to build up a new representation $\mathcal{M}_{\mathfrak{g}}$ of $\mathfrak{g}$. This procedure uses the natural action of $\mathfrak{g}$ on the universal enveloping superalgebra 
$\mathcal{U}(\mathfrak{g})$. More formally, one has

$$
\mathcal{M}_{\mathfrak{g}}=\operatorname{Ind}_{\mathfrak{k}}^{\mathfrak{g}}\left(\mathcal{M}_{\mathfrak{k}}\right) \stackrel{\text { def }}{=} \mathcal{U}(\mathfrak{g}) \otimes_{\mathfrak{k}} \mathcal{M}_{\mathfrak{k}}
$$

where the symbol $\otimes_{\mathfrak{k}}$ refers to the fact that the tensor product is transparent with regard to generators from $\mathfrak{k}$, i.e. $X Y \otimes_{\mathfrak{k}} Z=X \otimes_{\mathfrak{k}} Y Z$ for all $Y \in \mathfrak{k}$. Due to the infinite dimensionality of $\mathcal{U}(\mathfrak{g})$, induction generally gives rise to representations which are very large and not necessarily irreducible. The power of induction thus heavily relies on a smart choice of subalgebra $\mathfrak{k}$.

For Lie superalgebras, a variety of subalgebras have been suggested, each of them clarifying different aspects of the representation theory. Two of the examples that will be used in this paper are associated with the root space decomposition and the splitting of $\mathfrak{g}$ into its even and odd part

$$
\text { a) } \mathfrak{g}=\mathfrak{h} \oplus \bigoplus_{\alpha \neq 0} \mathfrak{g}_{\alpha} \quad \text { and } \quad \text { b) } \quad \mathfrak{g}=\mathfrak{g}_{\underline{0}} \oplus \mathfrak{g}_{\underline{1}} .
$$

While the root space decomposition a) paves the road to highest weight theory, the second decomposition b) has certain advantages with regard to disclosing the categorial properties of representations. Moreover, in the case of Lie superalgebras of type I and type II, respectively, it is custom to work with the distinguished $\mathbb{Z}$-gradings

$$
\text { c) } \quad \mathfrak{g}=\mathfrak{g}_{1} \oplus \mathfrak{g}_{0} \oplus \mathfrak{g}_{-1} \quad \text { and } \quad \text { d) } \quad \mathfrak{g}=\mathfrak{g}_{2} \oplus \mathfrak{g}_{1} \oplus \mathfrak{g}_{0} \oplus \mathfrak{g}_{-1} \oplus \mathfrak{g}_{-2}
$$

where it is understood that $\left[\mathfrak{g}_{i}, \mathfrak{g}_{j}\right] \subset \mathfrak{g}_{i+j}$ for all $i, j \in \mathbb{Z}$. In both cases, the grading is assumed to be consistent with the underlying $\mathbb{Z}_{2}$-grading in the sense that $\mathfrak{g}_{\text {even }} \subset \mathfrak{g}_{\underline{0}}$ and $\mathfrak{g}_{\text {odd }} \subset \mathfrak{g}_{\underline{1}}$. As we shall see below, the decomposition c) is actually the most convenient one for constructing irreducible representations. In contrast, the decomposition d) is only natural if one is interested in specific types of infinite dimensional representations.

Highest weight modules. Highest weight theory is the easiest route towards the classification of (finite dimensional) irreducible representations and for the determination of Casimir invariants. The starting point is a one-dimensional representation $\mathbb{C}_{\lambda}$ of the Cartan subalgebra $\mathfrak{h}$. It is fully specified in terms of a weight $\lambda \in \mathfrak{h}^{*}$ by the assignment $H \mapsto \lambda(H)$. This one-dimensional representation is then extended to a representation of the Borel subalgebra $\mathfrak{b}_{>}=\mathfrak{h} \oplus \bigoplus_{\alpha>0} \mathfrak{g}_{\alpha}$ by letting all positive roots act trivially 5 Finally, the negative roots are used to generate new states. This procedure results in the Verma module

$$
\mathcal{V}_{\lambda} \stackrel{\text { def }}{=} \operatorname{Ind}_{\mathfrak{b}>}^{\mathfrak{g}}\left(\mathbb{C}_{\lambda}\right)
$$

Verma modules are infinite dimensional as soon as there is at least one even root. Irreducible representations $\mathcal{L}_{\lambda}$ are recovered as the quotient $\mathcal{V}_{\lambda} / \mathcal{M}_{\lambda}$ of a Verma module by its maximal proper submodule $\mathcal{M}_{\lambda}$.

Verma modules have the advantage that they are easy to define and that their characters are trivial to write down. Also, the root space decomposition leads to a simple expression for the eigenvalue of the quadratic Casimir operator ( $\rho$ is the Weyl vector),

$$
C(\lambda)=(\lambda, \lambda+2 \rho)
$$

in terms of the naturally induced invariant form $(\cdot, \cdot)$ on $\mathfrak{h}^{*}$. On the other hand, the identification of the maximal submodule $\mathcal{M}_{\lambda}$ and the derivation of character formulas for the irreducible quotients $\mathcal{L}_{\lambda}$ is rather cumbersome.

Kac modules $\mathcal{K}_{\lambda}$. Kac modules are finite dimensional representations which have been designed to approximate irreducibility as closely as possible [49,50. The definition is rather involved for Lie superalgebras of type II. For this reason, we will restrict our attention to Lie superalgebras of type I

\footnotetext{
${ }^{5}$ It will not be important for our presentation, but we wish to remark that for Lie superalgebras there exist various choices of inequivalent simple root systems (and associated Dynkin diagrams). This is in stark contrast to the case of ordinary Lie algebras.
} 
here, where Kac modules are extremely well behaved. In this case, we can use the distinguished $\mathbb{Z}$-grading c) from eq. (2.14) in order to split the fermions into two dual spaces $\mathfrak{g}_{ \pm 1}$, each of which forms an irreducible representation of $\mathfrak{g}_{0}=\mathfrak{g}_{\underline{0}}$. It is then straightforward to extend any $\mathfrak{g}_{\underline{0}}$-module $L_{\lambda}$ to a representation of $\mathfrak{g}_{0} \oplus \mathfrak{g}_{1}$ by letting the odd generators from $\mathfrak{g}_{1}$ act trivially. Since the remaining fermions in $\mathfrak{g}_{-1}$ are all nilpotent, the induced module

$$
\mathcal{K}_{\lambda}=\operatorname{Ind}_{\mathfrak{g}_{0} \oplus \mathfrak{g}_{1}}^{\mathfrak{g}} L_{\lambda}=\mathcal{U}(\mathfrak{g}) \otimes_{\mathfrak{g}_{0} \oplus \mathfrak{g}_{1}} L_{\lambda}
$$

is finite dimensional. As a representation of $\mathfrak{g}_{\underline{0}}$ one immediately finds

$$
\left.\mathcal{K}_{\lambda}\right|_{\mathfrak{g}_{\underline{0}}}=L_{\lambda} \otimes \bigwedge\left(\mathfrak{g}_{-1}\right)
$$

and this expression leads to a straightforward formula for the character of $\mathcal{K}_{\lambda}$ [49, 50]. Just as for Verma modules, one can associate a simple module $\mathcal{L}_{\lambda}$ to every Kac module $\mathcal{K}_{\lambda}$ by taking the quotient $\mathcal{L}_{\lambda}=\mathcal{K}_{\lambda} / \mathcal{N}_{\lambda}$ by its maximal submodule $\mathcal{N}_{\lambda}$. It can be shown that all simple modules can be obtained this way. Dual Kac modules $\mathcal{K}_{\mu}^{*}$ are obtained by replacing $L_{\lambda}$ with its dual $L_{\lambda}^{*}$ and by exchanging the roles of $\mathfrak{g}_{ \pm 1}$.

Typical versus atypical representations. Kac modules are of particular relevance since they typically turn out to be irreducible. If this is the case, the associated simple module $\mathcal{L}_{\lambda}=\mathcal{K}_{\lambda}$ is called typical, otherwise atypical (this definition only applies to type I superalgebras) 6 There are different ways of characterizing whether a module $\mathcal{L}_{\lambda}$ is typical or atypical. For instance, a weight $\lambda$ is leading to a typical representation if there is an isotropic fermionic root $\alpha$ such that 50]

$$
(\lambda+\rho, \alpha)=0 .
$$

The root is called isotropic if $(\alpha, \alpha)=0$. One can even define the degree of atypicality $d(\lambda)$ by counting (in a suitable way) the solutions $\alpha$ to this equation (see, e.g., [51]). The larger $d(\lambda)$, the more complicated the representation $\mathcal{K}_{\lambda}$ and the resolution of $\mathcal{L}_{\lambda}$ in terms of Kac modules. The maximal value of $d(\lambda)$ when taken over all finite dimensional representations is known as the defect $d(\mathfrak{g})$ of the Lie superalgebra $\mathfrak{g}[46$. It is a measure for how pathological the representation theory of $\mathfrak{g}$ is. Lie superalgebras with $d(\mathfrak{g}) \leq 1$ are under good control (see also Table 1). In contrast, a defect $d(\mathfrak{g})>1$ indicates that the representation theory is of wild type, i.e. the classification of all finite dimensional representations (as opposed to merely irreducible representations) is believed to be beyond reach (see [52] for a detailed discussion in connection with $\operatorname{sl}(m \mid n)$ ).

One of the most fundamental problems in the representation theory of Lie superalgebras is to find explicit character formulas for atypical irreducible representations. Recent progress on this essential issue can be found in the references [53, 54, 55, 56, 57. In particular, the concept of "super duality" appears to be a very powerful new tool [58, 51]. For some Lie superalgebras of physical interest, most notably $\operatorname{sl}(n \mid 1), \operatorname{psl}(2 \mid 2)$ and $D(2,1 ; \alpha)$, it is also possible to consult more specific literature (see, e.g., [52, 59, 60, 61, 24 and references therein). In Section 2.3.2 we will introduce an alternative (but less explicit) characterization of typicality which is emphasizing category theoretic aspects.

Projective modules $\mathcal{B}_{\lambda}$. Let us next investigate induced representations which result from the decomposition b) of $\mathfrak{g}$. Inducing from an irreducible representation $L_{\lambda}$ of the even subalgebra $\mathfrak{g}_{\underline{0}}$ we obtain the modules

$$
\mathcal{B}_{\lambda}=\operatorname{Ind}_{\mathfrak{g}_{\underline{0}}}^{\mathfrak{g}}\left(L_{\lambda}\right)
$$

Even though they are too large to give rise to irreducible representations of $\mathfrak{g}$ directly, they have a number of very convenient properties. In particular, they are always finite dimensional (if $L_{\lambda}$ is) and they inherit the property of being projective from $L_{\lambda}$ (see below) since induction is a rightexact functor. As we shall discuss in Section 2.4, the modules $\mathcal{B}_{\lambda}$ play a key role in the harmonic analysis on supergroups.

\footnotetext{
${ }^{6}$ In physics terminology one would call them non-BPS and BPS representations or long and (semi-)short representations, respectively. Equality (2.19) defines the equivalent of a BPS bound.
} 
Characterization of indecomposable representations: Composition series. Indecomposable representations which are not irreducible can (partially) be characterized by means of composition series 7 By a composition series of an indecomposable module $\mathcal{M}$ we shall mean a filtration

$$
\{0\}=\mathcal{M}_{0} \subset \mathcal{M}_{1} \subset \cdots \subset \mathcal{M}_{n}=\mathcal{M}
$$

such that all quotient modules $\mathcal{Q}_{i}=\mathcal{M}_{i} / \mathcal{M}_{i-1}$ are simple, i.e. irreducible. A composition series can be obtained iteratively, by letting $\mathcal{M}_{n-1}$ be a maximal submodule of $\mathcal{M}=\mathcal{M}_{n}, \mathcal{M}_{n-2}$ be a maximal submodule of $\mathcal{M}_{n-1}$ and so on. The simple modules $\mathcal{Q}_{i}$ are known as composition factors. Their respective multiplicities are invariants of $\mathcal{M}$, i.e. they do not depend on the exact choice of composition series 62. In this paper, we will use the pictorial description

$$
\mathcal{M}: \quad \mathcal{Q}_{n} \rightarrow \mathcal{Q}_{n-1} \rightarrow \cdots \rightarrow \mathcal{Q}_{1}
$$

in order to visualize the composition series of indecomposable representations. Moreover, we will frequently work with a slight variant of the composition series, the so-called socle series where the quotient modules $\mathcal{Q}_{i}$ are maximal semi-simple (instead of merely being simple). In that case, $\mathcal{Q}_{n}$ is known as the head of the module $\mathcal{M}$, while $\mathcal{Q}_{1}$ is known as its socle.

It should be noted that there is also another variant of the previous definition, where the quotients are required to be direct sums of Kac modules. In that case one speaks about a Kac composition series. While every module admits a composition series, this is not necessarily so regarding Kac composition series (a trivial example being any simple module that is obtained as a non-trivial quotient of a Kac module).

The existence of composition series with regard to two specific sets of modules, so-called standard and co-standard modules, is the foundation of tilting theory and it leads to profound consequences. For type I Lie superalgebras, the role of standard and co-standard modules is played by Kac modules 63. Since we will not need it here, we refrain from being more explicit.

Example: Representations of $\mathbf{g l}(1 \mid 1)$. The bosonic subalgebra of $\mathrm{gl}(1 \mid 1)$ is spanned by $E$ and $N$ and is isomorphic to $\operatorname{gl}(1) \oplus \operatorname{gl}(1)$. At the same time, it forms the Cartan subalgebra of $\mathrm{gl}(1 \mid 1)$. Its simple modules are all one-dimensional and given by $L_{(e, n)}=\mathbb{C}_{(e, n)}$ where $e$ and $n$ are the eigenvalue of $E$ and $N$, respectively. Starting from $L_{(e, n)}$ one can induce the Kac module $\mathcal{K}_{(e, n)}$ of $\mathrm{gl}(1 \mid 1)$ by letting $\psi^{+}$act trivially on an arbitrary non-zero vector $|e, n\rangle$ of $L_{(e, n)}$. The resulting module is spanned by the two states $v_{1}=|e, n\rangle$ and $v_{2}=\psi^{-}|e, n\rangle$. The second vector has weight $(e, n-1)$. By applying $\psi^{+}$to $v_{2}$ one can easily verify that $\mathcal{K}_{(e, n)}$ is irreducible, $\mathcal{L}_{(e, n)}=\mathcal{K}_{(e, n)}$, if and only if $e \neq 0$. For $e=0$, on the other hand, $v_{2}$ spans an invariant submodule since $v_{1}$ cannot be reached anymore by the action of $\psi^{+}$. As a consequence, the module $\mathcal{K}_{(0, n)}$ has a composition series of length two which may be depicted as

$$
\mathcal{K}_{(0, n)}: \quad \mathcal{L}_{n} \rightarrow \mathcal{L}_{n-1},
$$

where $\mathcal{L}_{n}:=\mathcal{L}_{(0, n)}$ refers to the one-dimensional simple module on which $N$ acts as multiplication by $n$ and all the remaining generators, $E, \psi^{ \pm}$, act trivially. Finally, the modules $\mathcal{B}_{(e, n)}$ are obtained by acting on $L_{(e, n)}=\mathbb{C}_{(e, n)}$ with all possible fermionic operators. This gives rise to a fourdimensional space which decomposes as $\mathcal{B}_{(e, n)}=\mathcal{L}_{(e, n)} \oplus \mathcal{L}_{(e, n+1)}$ for $e \neq 0$. In contrast, for $e=0$, the module $\mathcal{B}_{(0, n)}$ turns out to be indecomposable with a socle series 8

$$
\mathcal{B}_{(0, n)}: \quad \mathcal{L}_{n} \rightarrow \mathcal{L}_{n+1} \oplus \mathcal{L}_{n-1} \rightarrow \mathcal{L}_{n}
$$

Actually, $\mathcal{B}_{(0,0)}$ just corresponds to the adjoint representation of $\operatorname{gl}(1 \mid 1)$.

In addition to the representations just mentioned, the Lie superalgebra $\mathrm{gl}(1 \mid 1)$ admits a whole zoo of finite dimensional indecomposable representations. The complete classification can be found in [60, including the full set of tensor product decompositions. Anticipating the notation $\mathcal{P}_{n}=$ $\mathcal{B}_{(0, n)}$, an illustration of the most important representations of $\mathrm{gl}(1 \mid 1)$ is provided in Figure 19

\footnotetext{
${ }^{7}$ Frequently, one also encounters the name "Jordan-Hölder series" 62 .

${ }^{8}$ Alternatively, it also has a Kac composition series $\mathcal{B}_{(0, n)}: \mathcal{K}_{(0, n)} \rightarrow \mathcal{K}_{(0, n+1)}$.

${ }^{9}$ These pictures should not be confused with the composition or socle series even though - in the particular case of $\operatorname{gl}(1 \mid 1)$, where the even subalgebra coincides with the Cartan subalgebra - these notions are closely related.
} 


$$
\begin{gathered}
\text { Typical sector }(E \neq 0) \\
\mathcal{L}_{(e, n)}=\mathcal{K}_{(e, n)}=\mathcal{P}_{(e, n)} \\
\bullet \begin{array}{c}
\bullet-1 \\
n
\end{array}
\end{gathered}
$$

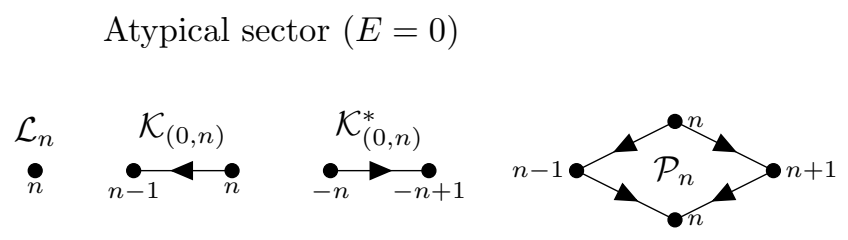

Figure 1: The weight diagrams of the most important representations of $g \mathrm{l}(1 \mid 1)$. The horizontal axis denotes the eigenvalues of $N$. The arrows indicate maps into invariant subspaces.

\subsubsection{Category theoretic perspective}

The previous section was mainly concerned with defining an interesting set of representations. Here we would like to clarify the relations betweens the modules $\mathcal{L}_{\lambda}, \mathcal{K}_{\lambda}$ and $\mathcal{B}_{\lambda}$ and bring some structure into the zoo of representations. This will be achieved by means of concepts from category theory, see 62 for a helpful reference. There are several categories of representations at our disposal. With regard to Verma modules, the most appropriate one is category $\mathcal{O}$ [64, 65, 51,. From the perspective of Kac modules, it is more natural to restrict one's attention to the category of representations with diagonalizable action of the Cartan subalgebra $\mathfrak{h}$. In particular, the previous category includes the category of finite dimensional weight modules over $\mathfrak{g}$ [66. All statements in this section refer to the latter 10

Much of the subsequent exposition is based on the concept of a projective module. A $\mathfrak{g}$-module $\mathcal{P}$ is called projective (in a given category of representations) if and only if for every surjective $\mathfrak{g}$-homomorphism $f: \mathcal{M} \rightarrow \mathcal{P}$ there exists a $\mathfrak{g}$-homomorphism $h: \mathcal{P} \rightarrow \mathcal{M}$ such that $f \circ h=$ id. In other words, in case $\mathcal{M}$ is a cover of $\mathcal{P}$ then it contains $\mathcal{P}$ as a direct summand and the map $f$ can be thought of as the projection onto $\mathcal{P}$. In the present context, i.e. restricting all considerations to the category of finite-dimensional $\mathfrak{g}$-modules, projective modules also satisfy the dual property of being injective [52]. One could then replace our previous definition by the requirement that any projective submodule $\mathcal{P}$ of an arbitrary module $\mathcal{M}$ always appears as a direct summand.

Let $\operatorname{SRep}(\mathfrak{g})$ denote the set of (equivalence classes of) all finite dimensional simple modules $\mathcal{L}_{\lambda}$. As we have seen in Section 2.3.1, elements from this set will be indexed by $\lambda$, with $\lambda$ running through some set of weights (a weight of $\mathfrak{g}$ and $\mathfrak{g}_{\underline{0}}$ at the same time). As we have reviewed in Section 2.3.1, the simple modules fall into two classes, typical and atypical representations. We split the set of weights accordingly into typical ones and atypical ones, $\operatorname{TRep}(\mathfrak{g})$ and $\operatorname{ARep}(\mathfrak{g})$. For finite-dimensional representations, it can be shown that the typical sector is characterized by the property that its simple modules are projective (in the sense described in the previous paragraph) while for the atypical representations this is not the case 11

To each weight $\lambda$ one can not only associate the simple module $\mathcal{L}_{\lambda}$ but rather also further indecomposable modules which contain $\mathcal{L}_{\lambda}$ is a simple quotient. Besides the Kac module $\mathcal{K}_{\lambda}$, the most important is the projective cover $\mathcal{P}_{\lambda}$ (see e.g. 63, Lemma 3.2]). The projective cover $\mathcal{P}_{\lambda}$ is the (unique) indecomposable projective module which has $\mathcal{L}_{\lambda}$ as a simple quotient. A representation $\mathcal{L}_{\lambda}$ is typical if and only if $\mathcal{L}_{\lambda} \cong \mathcal{P}_{\lambda}$, i.e. if $\mathcal{L}_{\lambda}$ is already projective itself. Otherwise $\mathcal{P}_{\lambda}$ is strictly larger than $\mathcal{L}_{\lambda}$. Every projective module may be decomposed into a direct sum of projective covers $\mathcal{P}_{\lambda}$ of simple modules. It should be noted that there are at least two independent intertwiners from $\mathcal{P}_{\lambda}$ to itself as long is $\lambda$ is atypical. The first one is the identity map. The other maps the head of $\mathcal{P}_{\lambda}$ to its socle and is thus nilpotent 12 In all examples we are aware of, the latter is given by a suitable power of the quadratic Casimir operator.

\footnotetext{
${ }^{10}$ Since we will later also be dealing with non-compact forms of supergroups associated to the Lie superalgebra $\mathfrak{g}$, some of our statements should be taken with a pinch of salt. To be precise, we will feel free to extrapolate results from a finite dimensional to an infinite dimensional setting where the corresponding mathematical results have not (yet) been rigorously established.

${ }^{11}$ According to 50, typical representations split in any finite-dimensional representation (this property may actually be taken as the definition of typicality). Hence they need to be their own projective cover.

${ }^{12}$ That the socle is equal to the head can be inferred from the equality $\operatorname{dim} \operatorname{Hom}_{\mathfrak{g}}\left(\mathcal{L}_{\lambda}, \mathcal{P}_{\lambda}\right)=\operatorname{dim} \operatorname{Hom}_{\mathfrak{g}}\left(\mathcal{P}_{\lambda}^{*}, \mathcal{L}_{\lambda}^{*}\right)=$ 1. The second equation just means that $\mathcal{L}_{\lambda}^{*}$ constitutes the head of $\mathcal{P}_{\lambda}^{*}$. To complete the argument one also has to use that the socle and the head do not sit on top of each other, i.e. that $\mathcal{P}_{\lambda}$ is not simple.
} 
After these preparations we are finally able to address the structure of the modules $\mathcal{B}_{\lambda}=$ $\operatorname{Ind}_{\mathfrak{g}_{0}}^{\mathfrak{g}}\left(L_{\lambda}\right)$ which are induced from finite dimensional simple $\mathfrak{g}_{\underline{0}}$-modules $L_{\lambda}$. Since $L_{\lambda}$ is projective and induction preserves this property, the module $\mathcal{B}_{\lambda}$ is projective as well (see, e.g., 66]). Hence it possesses a decomposition

$$
\mathcal{B}_{\lambda}=\bigoplus_{\mu \in \operatorname{SRep}(\mathfrak{g})} m_{\lambda \mu} \mathcal{P}_{\mu}
$$

into projective covers. Due to the relation $\operatorname{dim} \operatorname{Hom}_{\mathfrak{g}}\left(\mathcal{P}_{\lambda}, \mathcal{L}_{\mu}\right)=\delta_{\lambda \mu}$ ("the head of $\mathcal{P}_{\lambda}$ is $\mathcal{L}_{\lambda}$ "), the multiplicities can be obtained as the dimension

$$
m_{\lambda \mu}=\operatorname{dim} \operatorname{Hom}_{\mathfrak{g}}\left(\mathcal{B}_{\lambda}, \mathcal{L}_{\mu}\right)
$$

of a suitable space of intertwiners.

Finally, we introduce an equivalence relation on the set $\operatorname{SRep}(\mathfrak{g})$. Two weights $\mu$ and $\nu$ are said to be in the same block if there exists a non-split extension

$$
0 \rightarrow \mathcal{L}_{\mu} \rightarrow \mathcal{A} \rightarrow \mathcal{L}_{\nu} \rightarrow 0
$$

In other words, if $\mathcal{L}_{\mu}$ and $\mathcal{L}_{\nu}$ can be obtained as a submodule and a quotient of $\mathcal{A}$, respectively, but nevertheless $\mathcal{A}$ is not isomorphic to $\mathcal{L}_{\mu} \oplus \mathcal{L}_{\nu}$. The division of weights into blocks defines an equivalence relation which can be represented as a graph. The vertices are just the simple modules and two vertices are connected by a line if they admit a non-split extension. The blocks are the connected components of the resulting graph. There are a number of theorems on properties of blocks. For instance, it can be shown that the degree of atypicality is constant on blocks [52, 67, 51]. Moreover, different blocks are separated by central characters (i.e. the joint set of eigenvalues of all Casimir operators).

We will use the symbol $[\sigma]$ to denote the block a given weight $\sigma$ belongs to. A weight is typical if and only if the corresponding block contains precisely one element. The symbol ABlocks $(\mathfrak{g})$ will be reserved for the set of blocks obtained from atypical modules $\sigma$.

Example: The blocks of $\mathbf{g l}(\mathbf{1} \mid \mathbf{1})$. The simple representations of $\mathrm{gl}(1 \mid 1)$ fall into the blocks $[(e, n)]=\{(e, n)\}$ with $e \neq 0$ and $[n] \stackrel{\text { def }}{=}[(0, n)]=\{(0, n+m) \mid m \in \mathbb{Z}\}$, see the illustration

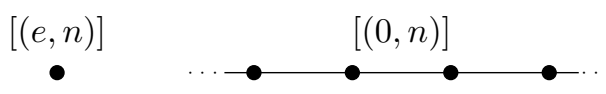

Indeed, the Kac module $\mathcal{K}_{(0, n)}$ provides a non-split extension

$$
0 \rightarrow \mathcal{L}_{n-1} \rightarrow \mathcal{K}_{(0, n)} \rightarrow \mathcal{L}_{n} \rightarrow 0
$$

The projective covers $\mathcal{P}_{n} \stackrel{\text { def }}{=} \mathcal{P}_{(0, n)}$ of atypical simple modules $\mathcal{L}_{n}=\mathcal{L}_{(0, n)}$ coincide $($ for gl $(1 \mid 1))$ with the modules $\mathcal{B}_{(0, n)}$. We note in passing that the quadratic Casimir operator $C$ acts in a non-diagonalizable fashion on the atypical projective covers $\mathcal{P}_{n}$ [31].

\subsection{Harmonic analysis on supermanifolds}

In this section, the harmonic analysis on supergroups and superspheres is discussed. In contrast to the purely bosonic case, the Laplacian turns out to be non-diagonalizable in the former case, thereby establishing a natural link to logarithmic conformal field theory. Our presentation will follow closely the logic of the article 68.

\subsubsection{Guide to the literature on supergeometry}

Roughly speaking, a supermanifold is a manifold which can locally be described by flat bosonic and fermionic - Grassmann algebra valued - coordinates. There are several competing definitions of how this physicist's intuition can be made mathematically precise. Since our main focus rests 
on physical applications, we are not capable of giving a complete account here, only a rather brief outline of some of the most important concepts. Readers interested in gaining a mathematically rigorous understanding of supermanifolds are invited to consult the original literature or some of the recent books on this subject. More physically minded readers may also start with Witten's recent exposition [69].

A very prominent approach follows the logic of Berezin, Kostant and Leites (BKL) and uses the language of algebraic geometry, in particular the concept of locally ringed spaces. As introductory books we can recommend [70, 71, 72, 73. Some more advanced aspects of the theory are treated in [74, 75. An alternative route, focusing more on differential geometric concepts and building up the theory from the local descriptions of supermanifolds, is covered in the books [76, 77, 78, 79. The two approaches give rise to equivalent notions of supermanifolds (in the sense of a categorical equivalence). At the level of computations in local coordinates, they are virtually identical. However, the BKL approach is more amenable to the application of advanced methods from algebraic geometry. Moreover, being more abstract, it is more flexible; for instance, only minor modifications lead to $\mathbb{Z}$-graded manifolds as they arise in mathematical treatments of the BV formalism.

\subsubsection{Supergroups}

A mathematically rigorous definition of a Lie supergroup $G$ can be obtained from a super HarishChandra pair $\left(\mathrm{G}_{\underline{0}}, \mathfrak{g}\right)$, i.e. a pair of an ordinary Lie group $\mathrm{G}_{\underline{0}}$ and a Lie superalgebra $\mathfrak{g}$ such that $\mathfrak{g}_{\underline{0}}$ is the Lie algebra associated with $\mathrm{G}_{\underline{\underline{0}}}$. Moreover, there should be an action of $\mathrm{G}_{\underline{0}}$ on $\mathfrak{g}$ which restricts to the adjoint action on $\mathfrak{g}_{\underline{0}}$ and whose differential close to the identity extends to the adjoint action of $\mathfrak{g}_{\underline{0}}$ on $\mathfrak{g}$ (see, e.g., $\overline{72}$ ).

In our review, we shall adopt a more ad-hoc perspective, thereby ignoring, to a large extent, potential mathematical subtleties. We shall simply assume that the idea of exponentiating a Lie algebra to obtain a Lie group carries over to Lie superalgebras if odd generators are paired with fermionic coordinates. For our purposes, a Lie supergroup $\mathrm{G}$ is then a fermionic extension of an ordinary Lie group $\mathrm{G}_{\underline{0}}$ by fermionic coordinates which transform in a suitable representation of $\mathrm{G}_{\underline{0}}$. All the properties of a supergroup are inherited from this data. For this reason, we will review the well-known harmonic analysis on ordinary compact Lie groups first. The goal of harmonic analysis is to learn about the structure of a group from studying the action of invariant differential operators - Lie derivatives and Laplace operators - on its algebra of functions.

Let us consider a compact, simple, simply-connected Lie group $\mathrm{G}_{\underline{\underline{0}}}$. According to the PeterWeyl theorem, the algebra $\mathcal{F}\left(\mathrm{G}_{\underline{0}}\right)$ of square integrable functions on $\mathrm{G}_{\underline{\underline{0}}}$ (with respect to the Haar measure) decomposes as

$$
\mathcal{F}\left(\mathrm{G}_{\underline{0}}\right) \cong \bigoplus_{\mu \in \operatorname{SRep}\left(\mathrm{G}_{\underline{0}}\right)} L_{\mu} \otimes L_{\mu}^{*}
$$

under the left-right regular action $(l, r) \cdot f: g \mapsto f\left(l^{-1} g r\right)$ of $\mathrm{G}_{\underline{0}} \times \mathrm{G}_{\underline{0}}$, where the sum is over all finite dimensional irreducible representations of $\mathrm{G}_{\underline{0}}$ and the asterisk $*$ refers to the dual representation. Each individual term in the decomposition can be thought of as being associated with representation matrices $\rho^{(\mu)}(g) \in \operatorname{End}\left(L_{\mu}\right)$ with $g \in \mathrm{G}_{\underline{0}}$. The statement of the Peter-Weyl theorem is that these matrix elements can be used to approximate any function on $\mathrm{G}_{\underline{0}}$ with arbitrary precision. In eq. (2.29) it has been assumed implicitly that we consider the closure of the right hand side.

The extension to supergroups is straightforward. Compared to an ordinary group, a supergroup comes with additional Grassmann algebra valued coordinates which generate the exterior algebra $\bigwedge\left(\mathfrak{g}_{1}^{*}\right)$. This space admits an obvious action of $\mathfrak{g}_{\underline{0}}$ by the Lie bracket. The algebra of functions on the supergroup $\mathrm{G}$ is the induced module (with respect to the right action of $\mathrm{G}_{\underline{0}}$ )

$$
\mathcal{F}(\mathrm{G})=\operatorname{Ind}_{\mathfrak{g}_{\underline{0}}}^{\mathfrak{g}} \mathcal{F}\left(\mathrm{G}_{\underline{0}}\right)=\mathcal{F}\left(\mathrm{G}_{\underline{0}}\right) \otimes \bigwedge\left(\mathfrak{g}_{\underline{1}}^{*}\right)
$$

This definition has a natural interpretation arising from formally expanding functions on $\mathrm{G}$ in a Taylor series in the odd coordinates. Apart from the right action of $G, \mathcal{F}(\mathrm{G})$ also admits a left action, just as in the bosonic case. Our goal is to understand the decomposition of this algebra 
as a $\mathfrak{g} \oplus \mathfrak{g}$-module (with respect to the left and right regular action). The result will provide a super-analogue of the Peter-Weyl theorem.

Since all finite dimensional representations of a reductive Lie algebra $\mathfrak{g}_{\underline{0}}$ are projective the same will be true for the induced module $\mathcal{F}(\mathrm{G})$. Hence, as a right $\mathfrak{g}$-module, $\mathcal{F}(\overline{\mathrm{G}})$ has the decomposition

$$
\mathcal{F}(\mathrm{G})=\bigoplus_{\mu \in \operatorname{SRep}(G)} M_{\mu} \otimes \mathcal{P}_{\mu}^{*}
$$

where the sum is over all projective covers of $\mathfrak{g}$ and the $M_{\mu}$ are some multiplicity spaces. As a left $\mathfrak{g}$-module, $\mathcal{F}(\mathrm{G})$ has precisely the same decomposition. Indeed, the algebra of functions has to be isomorphic with respect to the left and the right regular action due to the existence of the isomorphism $\Omega: \mathcal{F}(\mathrm{G}) \rightarrow \mathcal{F}(\mathrm{G})$ which acts as $\Omega(f): g \mapsto f\left(g^{-1}\right)$ and which intertwines the left and right regular actions.

In the typical sector, $\mathcal{P}_{\mu}$ agrees with $\mathcal{L}_{\mu}$. Given the symmetry between the left and the right action it is then obvious that $M_{\mu} \cong \mathcal{L}_{\mu}$ as vector spaces. We will now show that this is indeed always the case, not only in the typical sector but also in the atypical sector. First of all we notice that, by definition (2.20) and eq. (2.25), the algebra of functions on $\mathrm{G}$ has the form

$$
\mathcal{F}(\mathrm{G})=\bigoplus_{\mu \in \operatorname{SRep}\left(\mathfrak{g}_{\underline{0}}\right)} L_{\mu} \otimes \mathcal{B}_{\mu}^{*}=\bigoplus_{\mu \in \operatorname{SRep}\left(\mathfrak{g}_{\underline{0}}\right)} m_{\mu \nu} L_{\mu} \otimes \mathcal{P}_{\mu}^{*}
$$

as a $\mathfrak{g}_{\underline{0}} \oplus \mathfrak{g}$-module. We then employ Frobenius reciprocity to rewrite eq. (2.26) as

$$
m_{\mu \nu}=\operatorname{dim} \operatorname{Hom}_{\mathfrak{g}_{\underline{0}}}\left(L_{\mu}, \mathcal{L}_{\nu}\right),
$$

which proves our assertion, given that all $\mathfrak{g}_{\underline{0}}$-modules are fully reducible.

The result just obtained suggests that we have a factorization $\mathcal{L}_{\mu} \otimes \mathcal{L}_{\mu}^{*}$ of the individual contributions in the typical sector, just as in the case of $\mathcal{F}\left(\mathrm{G}_{\underline{0}}\right)$. In the atypical sector, however, such a factorization is not possible since the projective covers $\mathcal{P}_{\mu}$ are strictly larger than the simple modules $\mathcal{L}_{\mu}$. For this reason, the left and right modules in the atypical sector are entangled in a complicated way, arranging themselves in one infinite dimensional non-factorizable (non-chiral) indecomposable $\mathfrak{g} \oplus \mathfrak{g}$-module $\mathcal{I}_{[\sigma]}$ for each individual block $[\sigma]$. We finally find 13

$$
\mathcal{F}(\mathrm{G})=\bigoplus_{\mu \in \mathrm{TRep}(\mathfrak{g})} \mathcal{L}_{\mu} \otimes \mathcal{L}_{\mu}^{*} \oplus \bigoplus_{[\sigma] \in \operatorname{ABlocks}(\mathfrak{g})} \mathcal{I}_{[\sigma]}
$$

for the decomposition of $\mathcal{F}(\mathrm{G})$ as a $\mathfrak{g} \oplus \mathfrak{g}$-module. For Lie supergroups of type I an alternative derivation based on BGG duality [66] has been worked out in great detail in [38. In that case, the distinguished $\mathbb{Z}$-grading (2.14) allows to split the fermions into two separate sets which transform non-trivially only under the left and right action of $\mathrm{G}_{\underline{0}}$ on $\mathrm{G}$, respectively. Needless to say, this decomposition is perfectly adapted to the natural bimodule structure of $\mathcal{F}(G)$.

It should be emphasized that the Laplace operator $\Delta$ - which can be thought of as half the quadratic Casimir operator acting on $\mathcal{F}(\mathrm{G})$ - is not diagonalizable on the atypical projective covers $\mathcal{P}_{\mu}$ and on the non-chiral modules $\mathcal{I}_{[\sigma]}$, certainly for type I supergroups 38] but probably beyond. For $\sigma$-models on supergroups this implies that they are generally logarithmic conformal field theories (see 36 for an explicit derivation of logarithmic correlation functions). Exceptions may occur for small volumes where the spectrum of the CFT can be truncated in such a way that the modules $\mathcal{I}_{[\sigma]}$ no longer contribute [37, 39].

Example: Harmonic analysis on GL(1|1). Since our previous result has been very abstract, let us explain it in more detail using the example of GL(1|1) (first considered in 36]). As coordinates we choose $x, y, \eta, \bar{\eta}$ which are used to represent a general group element $g \in \mathrm{GL}(1 \mid 1)$ in the form

$$
g=e^{i \eta \psi^{+}} e^{i x E+i y N} e^{i \bar{\eta} \psi^{-}} .
$$

\footnotetext{
${ }^{13}$ Versions of this result appear to be known among mathematicians specialized on Lie superalgebras, even though no specific reference seems to exist. In the physics literature, the result was first noted in 38 and, from the more general perspective adopted here, in [68].
} 

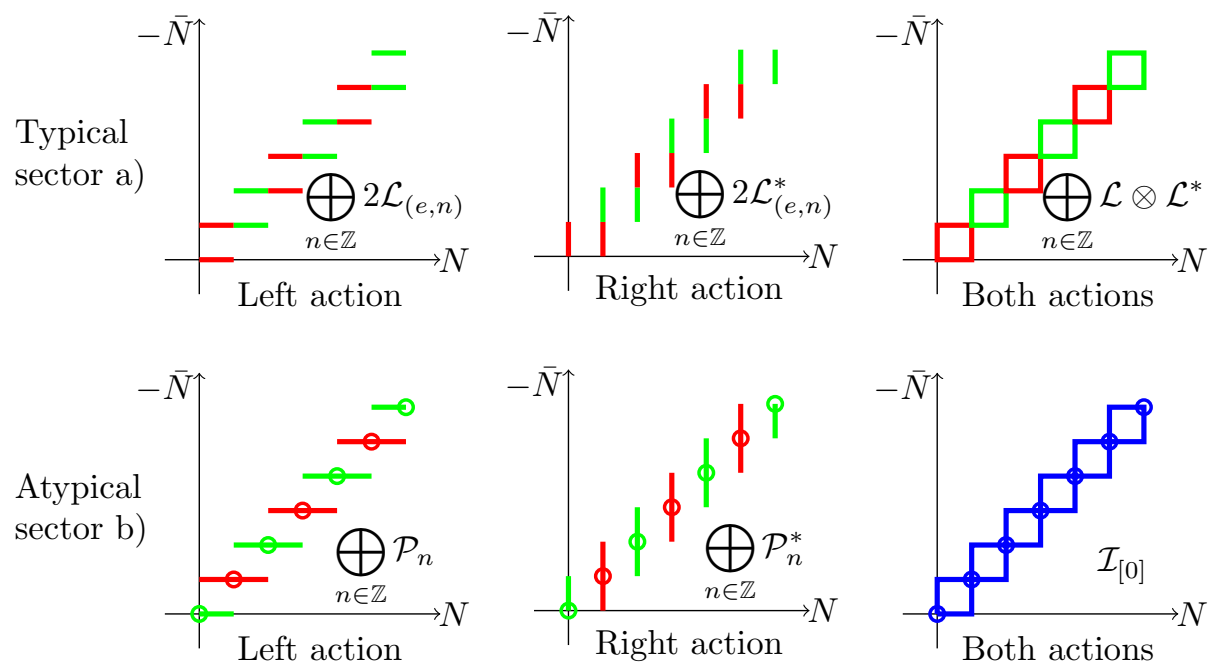

Figure 2: (Color online) Sketch of the harmonic analysis on supergroups using the example of GL(1|1). It is shown how the space of functions organizes itself with respect to the left and the right action of $\mathfrak{g}$ (the axes correspond to eigenvalues of the respective Cartan subalgebra) and with respect to the simultaneous action of both. In the typical sector a) we observe a factorization of representation (green and red) while in the atypical sector b) the functions organize themselves in infinite dimensional non-factorizing representations (blue) due to the extension of simple modules into projective covers.

The space of functions on GL(1|1) is spanned by the plane waves $e^{-i(e x+n y)}$, multiplied by a polynomial in the fermionic coordinates $\eta, \bar{\eta}$. On this space, the regular action of GL(1|1) on itself leads to the following two mutually (graded) commuting copies of the Lie superalgebra gl(1|1),

$$
\begin{array}{llll}
E=i \partial_{x}, & N=i \partial_{y}-\eta \partial_{\eta}, & \psi^{+}=-i \partial_{\eta}, & \psi^{-}=i e^{i y} \partial_{\bar{\eta}}-\eta \partial_{x} \\
\bar{E}=-i \partial_{x}, & \bar{N}=-i \partial_{y}+\bar{\eta} \partial_{\bar{\eta}}, & \bar{\psi}^{-}=-i \partial_{\bar{\eta}}, & \bar{\psi}^{+}=i e^{i y} \partial_{\eta}-\bar{\eta} \partial_{x} .
\end{array}
$$

We note that the generators $E, \bar{E}$ and $N, \bar{N}$ reduce to momentum operators when restricted to purely bosonic functions. The space of functions on $\mathrm{GL}(1 \mid 1)$ is spanned by the following vectors with quantum numbers

\begin{tabular}{c|cccc} 
& $e^{-i(e x+n y)}$ & $e^{-i(e x+n y)} \eta$ & $e^{-i(e x+n y)} \bar{\eta}$ & $e^{-i(e x+(n+1) y)} \eta \bar{\eta}$ \\
\hline \hline$(E, \bar{E})$ & $(e,-e)$ & $(e,-e)$ & $(e,-e)$ & $(e,-e)$ \\
$(N, \bar{N})$ & $(n,-n)$ & $(n-1,-n)$ & $(n,-n+1)$ & $(n,-n)$
\end{tabular}

Restricting our attention to integer values of $n$ and fixed value of $e$ for simplicity of illustration, the resulting weight diagram reduces to the patterns sketched in Figure 2. In the typical sector $e \neq 0$, the states organize themselves into two-dimensional simple modules $\mathcal{L}$, both with respect to the left and with respect to the right action (red and green lines). Under the combined action they combine into four-dimensional representations (red and green boxes) which correspond to the tensor product $\mathcal{L} \otimes \mathcal{L}^{*}$. In the atypical sector $e=0$, however, the picture is very different. Here the states organize themselves in four-dimensional projective covers $\mathcal{P}$ if only one of the two actions is considered. One should imagine the diamond from Figure 1 but now perpendicular to the plane, with two of the vertices being located in the plane. In this case the states cannot be organized in a tensor product under the combined action for obvious reasons. Instead, they have to combine into infinite dimensional indecomposable multiplets (blue), one for each value of $n \bmod 1$, i.e. one for each block.

Finally, we comment on the action of the Laplace operator $\Delta$. It is obtained by expressing the quadratic Casimir $C$ from (2.11) in terms of the Lie derivatives (2.36) and it reads

$$
\Delta=C / 2=-\partial_{x} \partial_{y}-i / 2 \partial_{x}-e^{i y} \partial_{\eta} \partial_{\bar{\eta}} \text {. }
$$


An explicit inspection of the two-dimensional $\Delta$-invariant subspace spanned by the functions $e^{-i(n+1)} \eta \bar{\eta}$ and $e^{-i n}$ shows that the Laplacian cannot be diagonalized on the atypical sector with $e=0$ (which is annihilated by $\partial_{x}$ ) 36 .

\subsubsection{Harmonic analysis on supercosets}

The harmonic analysis on a supercoset $\mathrm{G} / \mathrm{H}$ where the supergroup elements are identified according to the rule $g \sim g h$ with $h \in \mathrm{H}$ can be deduced from that of the supergroup case. Indeed, the algebra of functions on $\mathrm{G} / \mathrm{H}$ can be thought of as the space of $\mathrm{H}$-invariant functions on $\mathrm{G}$,

$$
\mathcal{F}(\mathrm{G} / \mathrm{H})=\operatorname{Inv}_{\mathrm{H}} \mathcal{F}(\mathrm{G}),
$$

where an element $h$ of the supergroup $\mathrm{H}$ acts on $f \in \mathcal{F}(\mathrm{G})$ according to

$$
h \cdot f(g)=f(g h) .
$$

It is obvious that the space $\mathrm{G} / \mathrm{H}$ and hence also the algebra of functions $\mathcal{F}(\mathrm{G} / \mathrm{H})$ still admits an action of $\mathrm{G}$. The isometry supergroup of $\mathrm{G} / \mathrm{H}$ might be bigger than $\mathrm{G}$ but for simplicity we will only consider the symmetry G.

Writing the invariant subspace (2.39) explicitly as a direct sum over indecomposable G-modules turns out to be rather involved in the general case. The main reason is that the modules over Lie superalgebras are not fully reducible. On the one hand such modules already appear in $\mathcal{F}(\mathrm{G})$, as G-modules with respect to the right regular action, see eq. (2.31). On the other hand, they may also arise when decomposing simple G-modules after restricting the action to the supergroup H. Finally, the invariants that need to be extracted when restricting from $\mathcal{F}(\mathrm{G})$ to $\mathcal{F}(\mathrm{G} / \mathrm{H})$ can be either true $\mathrm{H}$-invariants of $\mathcal{F}(\mathrm{G})$ (i.e. simple H-modules) or they can sit in a larger indecomposable $\mathrm{H}$-module. A general solution to this intricate problem is currently beyond reach. Nevertheless, eq. 2.32 teaches us one general lesson: In case $\mathrm{H}$ is purely bosonic, the algebra of functions $\mathcal{F}(\mathrm{G} / \mathrm{H})$ necessarily involves projective covers of simple G-modules, with a non-diagonalizable action of the Laplace operator. In particular, this observation is relevant for the supercosets describing AdS backgrounds in string theory.

The problem sketched in the previous paragraph can be circumvented when working on the level of characters since these are not sensitive to the indecomposable structure of modules. For many purposes this will be sufficient, as the following example shows.

Example: The supersphere $\mathbf{S}^{3 \mid 2}$. Instead of treating the general case, we discuss one example in some detail, namely the supersphere $\mathrm{S}^{3 \mid 2}$. This supersphere can be thought of as being embedded into the flat superspace $\mathbb{R}^{4 \mid 2}$, i.e. we have four bosonic coordinates $x^{i}$ and two fermionic coordinates $\eta_{1}, \eta_{2}$ subject to the constraint $\vec{x}^{2}+2 \eta_{1} \eta_{2}=R^{2}$. For our purposes, we can identify the algebra of functions $\mathcal{F}\left(\mathrm{S}^{3 \mid 2}\right)$ with the polynomial algebra $S\left(\mathbb{R}^{4 \mid 2}\right)$ in the six coordinates $\left(X^{a}\right)=\left(x^{i}, \eta_{\nu}\right)$ modulo the ideal generated by $\vec{X}^{2}=\vec{x}^{2}+2 \eta_{1} \eta_{2}=R^{2}$.

These coordinates transform in the vector representation of $\mathrm{SO}(4)$ and the defining representation of SP(2), respectively. Moreover, such bosonic transformations leave the constraint invariant. In addition, one can consider transformations which mix bosons and fermions. The resulting supergroup of isometries is $\operatorname{OSP}(4 \mid 2)$. Since the stabilizer of an arbitrary point on $\mathrm{S}^{3 \mid 2}$ is $\operatorname{OSP}(3 \mid 2)$, this confirms that the supersphere possesses a representation as a supercoset $\mathrm{OSP}(4 \mid 2) / \mathrm{OSP}(3 \mid 2)$.

In order to determine the character for the $\operatorname{OSP}(4 \mid 2)$-module $\mathcal{F}\left(\mathrm{S}^{3 \mid 2}\right)$ we proceed as follows. We first identify the Cartan subalgebra of $\operatorname{osp}(4 \mid 2)$ with the Cartan subalgebra of $\operatorname{su}(2) \oplus \operatorname{su}(2) \oplus \operatorname{su}(2)$, where we use the identifications $\operatorname{so}(4) \cong \operatorname{su}(2) \oplus \operatorname{su}(2)$ and $\operatorname{sp}(2) \cong \operatorname{su}(2)$. We then choose linear combinations of the six coordinates such that we can assign the weights $(\epsilon, \eta, 0)$ and $(0,0, \epsilon)$ to them, with $\epsilon, \eta= \pm 1$. In addition we introduce a quantum number for the polynomial grading. Each of the six coordinates $X^{a}$ then contributes a term $z_{1}^{m_{1}} z_{2}^{m_{2}} z_{3}^{m_{3}} t$ to the character, where $m_{i} \in\{0, \pm 1\}$ have to be chosen according to the respective weights and $t$ keeps track of the polynomial grade. For a product of coordinates, these individual contributions have to be multiplied with each other since the quantum numbers and the polynomial degree add up.

After these preparations it is very simple to write down the character of all polynomial functions in the coordinates $X^{a}$. Dividing out the ideal $\vec{X}^{2}=R^{2}$ is taken into account by multiplying the 
previous character with $1-t^{2}$ since the constraint relates every polynomial of degree $n \geq 2$ to a polynomial of degree $n-2$ with the same osp(4|2)-weight. At the end we take the limit $t \rightarrow 1$ since the grade is not a good quantum number once we impose the constraint. The total character is thus given by

$$
Z_{\mathcal{F}\left(\mathrm{S}^{3 \mid 2}\right)}\left(z_{1}, z_{2}, z_{3}\right)=\lim _{t \rightarrow 1} \frac{\left(1-t^{2}\right)\left(1+t z_{3}\right)\left(1+t / z_{3}\right)}{\left(1-t z_{1} z_{2}\right)\left(1-t z_{1} / z_{2}\right)\left(1-t z_{2} / z_{1}\right)\left(1-t / z_{1} z_{2}\right)}
$$

where all terms in the numerator should be expanded in a geometric series before the limit is taken [39]. The resulting expression can be represented as a linear combination of characters of osp $(4 \mid 2)$. Without going into the details we just write down the result

$$
Z_{\mathcal{F}\left(\mathrm{S}^{3 \mid 2}\right)}\left(z_{1}, z_{2}, z_{3}\right)=\chi_{[0,0,0]}\left(z_{1}, z_{2}, z_{3}\right)+\sum_{k=0}^{\infty} \chi_{[1 / 2, k / 2, k / 2]}\left(z_{1}, z_{2}, z_{3}\right)
$$

where the labels $\left[j_{1}, j_{2}, j_{3}\right]$ refer to simple modules (see [39]). Since all labels correspond to atypical irreducible representations in different blocks, this character decomposition at the same time yields the result for the harmonic analysis on $\mathrm{S}^{3 \mid 2}$ (see also [80]). The considerations of this section will be used in Section 4.3 when we discuss the spectrum of freely moving open strings on $\mathrm{S}^{3 \mid 2}$.

\subsection{Cohomological reduction}

We shall now review a tool which is capable of extracting part of the information of interest from a simplified setup. In the context of (logarithmic) conformal field theory it can be used to reduce the calculation of certain observables to those in theories of either free bosons or symplectic fermions 81. The method has been applied successfully to a non-perturbative proof of conformal invariance for symmetric superspace $\sigma$-models [81]. Combining it with the idea of quasi-abelian perturbation theory, see Section 3.5, it has also been used to conjecture exact D-brane spectra on projective superspaces [82].

\subsubsection{General formalism}

The procedure of cohomological reduction starts with the choice of a BRST charge $Q$ in the Lie superalgebra $\mathfrak{g}$. By definition, the BRST charge is a fermionic element $Q \in \mathfrak{g}$ which satisfies $[Q, Q]=0$. The existence of such a $Q$ is guaranteed for all Lie superalgebras for which atypical representations exist, e.g. for all simple Lie superalgebras except for $\operatorname{osp}(1 \mid 2 n)$. It implies the existence of a nilpotent operator $Q_{\mathcal{M}}=\rho_{\mathcal{M}}(Q)$ in any representation $\rho_{\mathcal{M}}: \mathfrak{g} \rightarrow \operatorname{End}(\mathcal{M})$. The equation $Q_{\mathcal{M}}^{2}=0$ then allows us to define the cohomology classes

$$
\mathrm{H}_{Q}(\mathcal{M})=\operatorname{Ker} Q_{\mathcal{M}} / \operatorname{Im} Q_{\mathcal{M}}
$$

Before we proceed with analyzing the general structure of the cohomology classes $\mathrm{H}_{Q}(\mathcal{M})$ it is useful and instructive to focus on the adjoint representation $\mathcal{M}=\mathfrak{g}$ for a second. Using just the Jacobi identity (2.1), it is then possible to establish that 81

1) the subspaces Ker $\operatorname{ad}_{Q}$ and $\operatorname{Imad}_{Q}$ are subalgebras of $\mathfrak{g}$,

2) the subalgebra $\operatorname{Imad}_{Q}$ is an ideal of $\operatorname{Ker} \operatorname{ad}_{Q}$,

3) the quotient space $\mathrm{H}_{Q}(\mathfrak{g})$ is a Lie superalgebra.

The element $Q$ thus defines a decomposition of $\mathfrak{g}$ into three vector superspaces $\mathfrak{h}, \mathfrak{e}, \mathfrak{f}$,

$$
\begin{aligned}
\mathfrak{g}=\mathfrak{h} \oplus \mathfrak{e} \oplus \mathfrak{f} \quad \text { such that } \\
\mathfrak{e}=\operatorname{Imad}_{Q}, \quad \mathfrak{h} \oplus \mathfrak{e}=\operatorname{Ker}_{Q} \quad \text { and } \quad \mathfrak{h}=\mathrm{H}_{Q}(\mathfrak{g}) .
\end{aligned}
$$

Moreover, any metric $\langle\cdot, \cdot\rangle$ on $\mathfrak{g}$ restricts to a non-degenerate form on $\mathfrak{h} \subset \mathfrak{g}$. The Lie subsuperalgebras $\mathfrak{e}$ and $\mathfrak{f}$, on the other hand, are isotropic, i.e. $\langle\mathfrak{e}, \mathfrak{e}\rangle=0=\langle\mathfrak{f}, \mathfrak{f}\rangle$. 
We also note that $\mathfrak{e}$ and $\mathfrak{f}$ both carry an action of the Lie superalgebra $\mathfrak{h}$. In other words, the direct sum decomposition of $\mathfrak{g}$ in eq. (2.44) can be regarded as an isomorphism of $\mathfrak{h}$-modules. The Lie superalgebra $\mathfrak{h}$ has been computed for various choices of $\mathfrak{g}$ and any $Q \in \mathfrak{g}$. The results may be summarized as follows

$$
\begin{aligned}
\mathfrak{h}(\operatorname{gl}(M \mid N)) & \simeq \operatorname{gl}\left(M-r_{Q} \mid N-r_{Q}\right), \\
\mathfrak{h}(\operatorname{sl}(M \mid N)) & \simeq \operatorname{sl}\left(M-r_{Q} \mid N-r_{Q}\right), \\
\mathfrak{h}(\operatorname{osp}(R \mid 2 N)) & \simeq \operatorname{osp}\left(R-2 r_{Q} \mid 2 N-2 r_{Q}\right) .
\end{aligned}
$$

The answer depends on $Q$ only through an integer $r_{Q} \geq 1$ that can be looked up in [81]. In all three cases listed above, there exist elements $Q$ with minimal rank $r_{Q}=1$.

The relevance of our previous discussion stems from the fact that all the linear spaces $\mathrm{H}_{Q}(\mathcal{M})$ come equipped with an action of the Lie sub-superalgebra $\mathfrak{h} \subset \mathfrak{g}$. First, notice that there is an $\mathfrak{h}$-stable filtration

$$
\mathcal{M} \supset \operatorname{Ker} Q_{\mathcal{M}} \supset \operatorname{Im} Q_{\mathcal{M}} .
$$

Indeed, $\mathcal{M}$ is an $\mathfrak{h}$-module by restriction, while $\operatorname{Ker} Q_{\mathcal{M}}$ and $\operatorname{Im} Q_{\mathcal{M}}$ are $\mathfrak{h}$-submodules because $\mathfrak{h} \subset \operatorname{Ker} \operatorname{ad}_{Q}$. Finally, $\operatorname{Ker} Q_{\mathcal{M}} \supset \operatorname{Im} Q_{\mathcal{M}}$ follows from $Q_{\mathcal{M}}^{2}=0$.

It is not difficult to see that $\mathcal{M} \rightarrow \mathrm{H}_{Q}(\mathcal{M})$ is functorial, i.e. it is consistent with forming tensor products, direct sums and conjugation in the category of $\mathfrak{h}$-modules [81. Even though $\mathrm{H}_{Q}(\mathcal{M})$ vanishes for a large class of representations, it can certainly contain non-trivial elements. A trivial example is the cohomology of the adjoint $\mathfrak{g}$-module $\mathcal{M}=\mathfrak{g}$ which reduces to $\mathrm{H}_{Q}(\mathfrak{g})=\mathfrak{h}$. For a finite dimensional $\mathfrak{g}$-module $\mathcal{M}$ one may actually show that

$$
\operatorname{sdim} \mathrm{H}_{Q}(\mathcal{M})=\operatorname{sdim} \mathcal{M}
$$

We conclude that all modules $\mathcal{M}$ with non-vanishing superdimension give rise to a non-trivial cohomology group $\mathrm{H}_{Q}(\mathcal{M}) \neq 0$. The condition $\operatorname{sim} \mathcal{M} \neq 0$ is often satisfied for atypical representations (short multiplets) and it is these representations that will survive the procedure of cohomological reduction. For typical representations $\mathcal{M}$, on the other hand, it can be proven that the cohomology $\mathrm{H}_{Q}(\mathcal{M})$ is always trivial. More generally, $\mathrm{H}_{Q}(\mathcal{M})=0$ for all (finite dimensional) projective modules. To summarize, each instance of cohomological reduction extracts information on certain atypical (short or semi-short) constituents of a given representation $\mathcal{M}$. In this regard it closely resembles supersymmetric indices.

\subsubsection{Application: Function spaces on supercosets}

Let us now discuss the application of cohomological reduction to supercosets $G / G^{\prime}$. For this purpose, we consider a Lie superalgebra $\mathfrak{g}$ along with a subalgebra $\mathfrak{g}^{\prime} \subset \mathfrak{g}$. The corresponding Lie supergroups will be denoted by $\mathrm{G}$ and $\mathrm{G}^{\prime}$, respectively. As before, we want to pick some fermionic element $Q \in \mathfrak{g}$ with $[Q, Q]=0$. Let us now assume that $Q$ is contained in the subalgebra $\mathfrak{g}^{\prime} \subset \mathfrak{g}$ so that its cohomology defines two Lie sub-superalgebras $\mathfrak{h} \subset \mathfrak{g}$ and $\mathfrak{h}^{\prime} \subset \mathfrak{g}^{\prime}$ with $\mathfrak{h}^{\prime} \subset \mathfrak{h}$. We denote the associated Lie supergroups by $\mathrm{H}$ and $\mathrm{H}^{\prime}$, respectively. Note that the space of functions on the coset superspace $\mathrm{G} / \mathrm{G}^{\prime}$ carries an action of $\mathfrak{g}$. In particular, the element $Q$ acts and gives rise to some cohomology. The central claim of this section is that the cohomology of some geometric object (smooth function, tensor form, square integrable function) defined on the coset superspace $\mathrm{G} / \mathrm{G}^{\prime}$ is equivalent to a similar object defined on $\mathrm{H} / \mathrm{H}^{\prime}$. This gives rise to isomorphisms of the type

$$
\mathrm{H}_{Q}\left(\mathcal{F}\left(\mathrm{G} / \mathrm{G}^{\prime}\right)\right) \cong \mathcal{F}\left(\mathrm{H} / \mathrm{H}^{\prime}\right)
$$

which means that the cohomology of $Q$ in the space of square integrable functions on $\mathrm{G} / \mathrm{G}^{\prime}$ may be interpreted as a space of square integrable functions on the coset superspace $\mathrm{H} / \mathrm{H}^{\prime}$. We note that $\mathcal{F}\left(\mathrm{H} / \mathrm{H}^{\prime}\right)$ carries an action of the Lie superalgebra $\mathfrak{h}=\mathrm{H}_{Q}(\mathfrak{g}) \subset \mathfrak{g}$. The isomorphism (2.48) is an isomorphism of $\mathfrak{h}$-modules. The derivation of eq. (2.48) is relatively involved and can be found in full detail in [81. 
Example: Cohomological reduction from $\mathbb{C P}^{\mathbf{1} \mid \mathbf{2}}$ to $\mathbb{R}^{\mathbf{0} 2}$. As an example of the above, let us discuss the Lie superalgebra $\mathfrak{g}=\operatorname{gl}(2 \mid 2)$. For $Q$ we pick the matrix that contains a single entry in the upper right corner. It is then easy to check that

$$
\operatorname{Ker}_{Q}=\mathfrak{h} \oplus \mathfrak{e} \ni\left(\begin{array}{cccc}
a_{11} & a_{12} & b_{11} & b_{12} \\
0 & a_{22} & b_{21} & b_{22} \\
0 & c_{12} & d_{11} & d_{12} \\
0 & 0 & 0 & a_{11}
\end{array}\right), \quad \operatorname{Im}_{Q} \mathfrak{g}=\mathfrak{e} \ni\left(\begin{array}{cccc}
a_{11} & a_{12} & b_{11} & b_{12} \\
0 & 0 & 0 & b_{22} \\
0 & 0 & 0 & d_{12} \\
0 & 0 & 0 & a_{11}
\end{array}\right) .
$$

Consequently, $\mathrm{H}_{Q}(\mathfrak{g})=\mathfrak{h}=\operatorname{gl}(1 \mid 1)$ consists of all supermatrices in which $a_{22}, b_{21}, c_{12}$ and $d_{11}$ are the only non-vanishing entries. Let us also specify the Lie sub-superalgebra $\mathfrak{g}^{\prime}$ to consist of all elements in $\mathfrak{g}$ with vanishing entries $b_{11}=b_{21}=d_{12}=d_{21}=c_{11}=c_{12}=0$. Hence, $\mathfrak{g}^{\prime} \cong \operatorname{gl}(2 \mid 1) \oplus \operatorname{gl}(1)$. The cohomology $\mathrm{H}_{Q}\left(\mathfrak{g}^{\prime}\right)=\mathfrak{h}^{\prime}=\operatorname{gl}(1) \oplus \operatorname{gl}(1)$ of $\mathfrak{g}^{\prime}$ can be read off easily.

In our example, the quotient $\mathrm{G} / \mathrm{G}^{\prime}$ is the complex projective superspace $\mathbb{C P}^{1 \mid 2} \cong S^{2} \times \mathbb{R}^{0 \mid 4}$. Functions thereon may be decomposed into finite dimensional representations of $\operatorname{psl}(2 \mid 2)$ as follows 82

$$
\mathcal{F}\left(\mathbb{C P}^{1 \mid 2}\right) \cong \bigoplus_{j=0}^{\infty} \mathcal{K}_{(j, 0)}
$$

The representations $\mathcal{K}_{(j, 0)}$ of $\operatorname{psl}(2 \mid 2)$ that appear in this decomposition possess dimension $d_{j}=$ $16(2 j+1)$. They are Kac modules generated from the spherical harmonics on the bosonic 2-sphere by application of four fermionic generators. For $j \neq 0$, the $\operatorname{psl}(2 \mid 2)$-modules $\mathcal{K}_{(j, 0)}$ turn out to be projective (typical long multiplets) and hence $\mathrm{H}_{Q}\left(\mathcal{K}_{(j, 0)}\right)=0$ for all $j \neq 0$. The only non-vanishing cohomology comes from the 16 -dimensional Kac module $\mathcal{K}_{(0,0)}$. The latter is built from three atypical irreducibles, namely two copies of the trivial representation $\mathcal{L}_{0}$ and one copy of the 14dimensional adjoint representation of $\operatorname{psl}(2 \mid 2)$ 61]. Each of these pieces contributes to cohomology. While the two trivial representations give rise to two even states, the adjoint representation has an excess of two odd states which descend to cohomology. In total, we obtain a 4-dimensional cohomology

$$
\mathrm{H}_{Q}\left(\mathcal{F}\left(\mathrm{G} / \mathrm{G}^{\prime}\right)\right)=\mathrm{H}_{Q}\left(\mathcal{F}\left(\mathbb{C P}^{1 \mid 2}\right)\right)=\mathrm{H}_{Q}\left(\mathcal{K}_{(0,0)}\right)=\mathbb{R}^{2 \mid 2}
$$

To be more precise, we note that the linear space $\mathbb{R}^{2 \mid 2}$ carries the 4 -dimensional projective cover of $\mathrm{gl}(1 \mid 1)$. According to our general statement, the cohomology should agree with the space of functions on the quotient $\mathrm{H} / \mathrm{H}^{\prime}=\mathrm{GL}(1 \mid 1) / \mathrm{GL}(1) \times \mathrm{GL}(1)$. The quotient possesses two fermionic coordinates and hence gives rise to a 4-dimensional algebra of functions over it,

$$
\mathcal{F}\left(\mathrm{H} / \mathrm{H}^{\prime}\right)=\mathbb{R}^{2 \mid 2}
$$

It indeed agrees with the cohomology in the space of functions over $\mathbb{C P}^{1 \mid 2}$, as it was claimed in eq. (2.48).

\section{Supergroup WZW models}

In this section we discuss supergroup WZW models as primary examples of logarithmic conformal field theories, emphasizing the connections to harmonic analysis. In the first part we focus on supergroups of type I. In this case, bosonic and fermionic degrees can be essentially decoupled, leading to the notion of a free fermion realization. As an example for the type II families we introduce the free field realization of the orthosymplectic series at level $k=1$.

\subsection{The action functional}

Let us fix a supergroup G together with a metric $\langle\cdot, \cdot\rangle$ of its Lie superalgebra $\mathfrak{g}$. We assume the supergroup to be simply-connected and the invariant form to be suitably normalized (see below). 
The supergroup WZW model is a two-dimensional $\sigma$-model describing the propagation of strings on $\mathrm{G}$. The action functional for maps $g: \Sigma \rightarrow \mathrm{G}$ is given by 83 ]

$$
\mathcal{S}^{\mathrm{WZW}}[g]=-\frac{i}{4 \pi} \int_{\Sigma}\left\langle g^{-1} \partial g, g^{-1} \bar{\partial} g\right\rangle d z \wedge d \bar{z}-\frac{i}{24 \pi} \int_{B_{3}}\left\langle g^{-1} d g,\left[g^{-1} d g, g^{-1} d g\right]\right\rangle .
$$

The second term is integrated over an auxiliary three-manifold $B_{3}$ which satisfies $\partial B_{3}=\Sigma$. Note that the measure $i d z \wedge d \bar{z}$ is real. The topological ambiguity of the second term possibly imposes a quantization condition on the metric $\langle\cdot . \cdot\rangle$ or, more precisely, on its bosonic restriction, in order to render the path integral well-defined 14

By construction, every WZW model has a global symmetry $\mathrm{G} \times \mathrm{G}$ corresponding to multiplying the field $g(z, \bar{z})$ by arbitrary group elements from the left and from the right. In fact, this symmetry is elevated to a current superalgebra symmetry $\hat{\mathfrak{g}}$

$$
J^{\mu}(z) J^{\nu}(w)=\frac{\left\langle J^{\mu}, J^{\nu}\right\rangle}{(z-w)^{2}}+\frac{i f_{\lambda}^{\mu \nu} J^{\lambda}(w)}{z-w}+\text { non-singular }
$$

and a corresponding anti-holomorphic symmetry if one allows these elements to depend holomorphically and antiholomorphically on $z$, respectively. In the last formula, the symbols $f$ denote the structure constants of $\mathfrak{g}$ and the currents are defined by $J \propto-\partial g g^{-1}$ and $\bar{J} \propto g^{-1} \bar{\partial} g$. The equations of motion guarantee that they are holomorphic and antiholomorphic, respectively.

Just as for bosonic WZW models, the current algebra (3.2) implies the existence of a conformal energy-momentum tensor which is obtained by the standard Sugawara construction. If $\mathfrak{g}$ is simple then the metric is uniquely determined up to a scale factor, the level $k$. In that case, the central charge of the WZW model is given by

$$
c=\frac{k \operatorname{sdim}(\mathrm{G})}{k+g^{\vee}},
$$

where $g^{\vee}$ is the dual Coxeter number (see Table 1) and $\operatorname{sdim} G=\operatorname{dim}\left(\mathfrak{g}_{0}\right)-\operatorname{dim}\left(\mathfrak{g}_{1}\right)$ is the superdimension of $\mathfrak{g}$. Since our treatment is supposed to cover simple as well as non-simple Lie supergroups, we will refrain from working with the level from now on.

One peculiarity of supergroup WZW models is that the indefinite signature of the metric implies that one part of the bosonic subgroup should be regarded as non-compact while the other one should be regarded as compact. One of the most familiar examples is the WZW model based on PSU(2|2) which features two SU(2) WZW models at positive and negative level, respectively. The most natural interpretation here corresponds to a supersymmetrization of the $\mathrm{SL}(2, \mathbb{R}) \times \mathrm{SU}(2)$ WZW model (corresponding to PSU $(1,1 \mid 2)$ ). An exception only occurs for small values of the level where quantum renormalization might help to bring a small negative level into the positive regime. In this case, supergroup WZW models may be used to describe a compact target space. An example of this type will be discussed in Section 4.3

\subsection{Free fermion realization for type I supergroups}

In order to unravel the peculiarities of supergroup WZW models we first of all concentrate on the special case of Lie supergroups of type I. These are singled out by the fact that they admit a distinguished $\mathbb{Z}$-grading (2.14). This grading permits to split the fermionic generators into two sets of generators $S_{1}^{a}$ and $S_{2 b}(a, b=1, \ldots, r)$ which are dual in the sense that $\left\langle S_{1}^{a}, S_{2 b}\right\rangle=\delta_{b}^{a}$. Moreover we assume that the bosonic generators $K^{i}$ give rise to a metric $\left\langle K^{i}, K^{j}\right\rangle=\kappa^{i j}$ of $\mathfrak{g}_{0}$. Under these circumstances, the complete set of non-trivial relations can be chosen to read 38 .

$$
\begin{aligned}
{\left[K^{i}, K^{j}\right] } & =i f^{i j}{ }_{l} K^{l}, & {\left[K^{i}, S_{1}^{a}\right] } & =-\left(R^{i}\right)_{b}^{a}{ }_{b}^{b}, \\
{\left[S_{1}^{a}, S_{2 b}\right] } & =-\left(R^{i}\right)^{a}{ }_{b} \kappa_{i j} K^{j}, & {\left[K^{i}, S_{2 a}\right] } & =S_{2 b}\left(R^{i}\right)^{b}{ }_{a} .
\end{aligned}
$$

\footnotetext{
${ }^{14}$ Note that for WZW models based on bosonic groups one usually explicitly introduces an integer valued constant, the level, which appears as a prefactor of the Killing form. For supergroups the Killing form might vanish. Hence there is no canonical normalization of the metric. Moreover, we would like to include models whose metric renormalizes non-multiplicatively (see below). Under these circumstances it is not particularly convenient to display the level explicitly and we assume instead that all possible parameters are contained in the metric $\langle\cdot, \cdot\rangle$.
} 
We note that the fermions $S_{1}^{a}$ and $S_{2 a}$ transform in an $r$-dimensional representation $R$ of $\mathfrak{g}_{\underline{0}}$ and its dual $R^{*}$, respectively. The symbol $R^{i}$ is an abbreviation for the corresponding representation matrix $R\left(K^{i}\right)$. The structure constants which appear in the commutator $\left[S_{1}^{a}, S_{2 b}\right]$ are uniquely determined by the requirement that the metric $\langle\cdot, \cdot\rangle$ is invariant.

Given the Lie superalgebra $\mathfrak{g}$, we can combine its generators with elements of a (suitably large) Grassmann algebra in order to obtain a Lie algebra which can be exponentiated. Following our previous strategy for GL(1|1), we shall define the supergroup G to be given by elements

$$
g=e^{\theta} g_{B} e^{\bar{\theta}}
$$

with $\theta=\theta^{a} S_{2 a}$ and $\bar{\theta}=\bar{\theta}_{b} S_{1}^{b}$. This particular way of distributing the fermions facilitates the decoupling of bosons and fermions and, at the same time, holomorphic factorization. The parametrization has been termed "chiral superspace" in 84. The coefficients $\theta^{a}$ and $\bar{\theta}_{b}$ are independent Grassmann variables while $g_{B}$ denotes an element of the bosonic subgroup $\mathrm{G}_{\underline{0}} \subset \mathrm{G}$ obtained by exponentiating the Lie algebra generators $K^{i}$. The attentive reader may have noticed that the product of two such supergroup elements (3.6) will not again give a supergroup element of the same form. We shall close an eye on such issues. For us, passing through the supergroup is merely an auxiliary step that serves the purpose of constructing a WZW-like conformal field theory with Lie superalgebra symmetry. Since Lie superalgebras do not suffer from problems with Grassmann variables, the resulting conformal field theory will be well-defined.

Given the parametrization (3.6), the Lagrangian (3.1) can be simplified considerably by making iterative use of the Polyakov-Wiegmann identity

$$
\mathcal{S}^{\mathrm{WZW}}[g h]=\mathcal{S}^{\mathrm{WZW}}[g]+\mathcal{S}^{\mathrm{WZW}}[h]-\frac{i}{2 \pi} \int\left\langle g^{-1} \bar{\partial} g, \partial h h^{-1}\right\rangle d z \wedge d \bar{z} .
$$

The WZW action evaluated on the individual fermionic bits vanishes because the invariant form is only supported on grade 0 of the $\mathbb{Z}$-grading. The final result is then

$$
\mathcal{S}^{\mathrm{WZW}}[g]=\mathcal{S}^{\mathrm{WZW}}\left[g_{B}, \theta\right]=\mathcal{S}^{\mathrm{WZW}}\left[g_{B}\right]-\frac{i}{2 \pi} \int\left\langle\bar{\partial} \theta, g_{B} \partial \bar{\theta} g_{B}^{-1}\right\rangle d z \wedge d \bar{z}
$$

For the correct determination of the mixed bosonic and fermionic term it was again necessary to refer to the grading of $\mathfrak{g}$.

It is now crucial to realize (see also [36, 24]) that we may pass to an equivalent first order description of the WZW model above by introducing an additional set of auxiliary fields $p_{a}$ and $\bar{p}^{a}$,

$$
\begin{aligned}
\mathcal{S}\left[g_{B}, p, \theta\right] & =\mathcal{S}_{\text {ren }}^{\mathrm{WZW}}\left[g_{B}\right]+\mathcal{S}_{\text {free }}[\theta, \bar{\theta}, p, \bar{p}]+\mathcal{S}_{\text {int }}\left[g_{B}, p, \bar{p}\right] \\
& =\mathcal{S}_{\text {ren }}^{\mathrm{WZW}}\left[g_{B}\right]+\frac{i}{2 \pi} \int\left\{\langle p, \bar{\partial} \theta\rangle-\langle\bar{p}, \partial \bar{\theta}\rangle-\left\langle p, g_{B} \bar{p} g_{B}^{-1}\right\rangle\right\} d z \wedge d \bar{z} .
\end{aligned}
$$

Here, $\theta, \bar{\theta}$ and our new fermionic fields $p=p_{a} S_{1}^{a}$ and $\bar{p}=\bar{p}^{a} S_{2 a}$ all take values in the Lie superalgebra $\mathfrak{g}$. Our conventions may look slightly asymmetric but as we will see later this just resembles the asymmetry in the parametrization (3.6). Up to certain subtleties that are encoded in the subscript "ren" of the first term, it is straightforward to see that we recover the original Lagrangian (3.8) upon integrating out the auxiliary fields $p$ and $\bar{p}$.

Let us comment a bit more on each term in the action (3.9). Most importantly, we need to specify the renormalization of the bosonic WZW model which results from the change in the path integral measure (cf. 85]). The computation of the relevant Jacobian has two important effects. First of all, it turns out that the construction of the purely bosonic WZW model entering the action (3.9) employs the following renormalized metric 15

$$
\left\langle K^{i}, K^{j}\right\rangle_{\text {ren }}=\kappa^{i j}-\gamma^{i j} \quad \text { with } \quad \gamma^{i j}=\operatorname{tr}\left(R^{i} R^{j}\right) .
$$

Note that this renormalization is not necessarily multiplicative. Only for simple Lie algebras the renormalized metric would be identical to the original one up to a factor, allowing for the familiar

\footnotetext{
${ }^{15}$ We assume this metric to be non-degenerate. Otherwise we would deal with what is known as the critical level or, in string terminology, the tensionless limit.
} 
interpretation as a level shift. For the bosonic subalgebra $\mathfrak{g}_{\underline{0}}$ of a basic Lie superalgebra $\mathfrak{g}$, however, this is not the case. First of all, the simple constituents of $\mathfrak{g}_{0}$ all come with their own level and each of them may be shifted differently. But then there are also examples such as $\operatorname{gl}(m \mid n)$ where $\mathfrak{g}_{\underline{0}}$ is not reductive and hence the notion of levels is not well-defined.

As a second consequence of the renormalization, the action (3.9) may contain a Fradkin-Tseytlin term, coupling a non-trivial dilaton to the world-sheet curvature $R^{(2)}$,

$$
\mathcal{S}_{\mathrm{FT}}^{\mathrm{WZW}}\left[g_{b}\right]=\int_{\Sigma} d^{2} \sigma \sqrt{h} R^{(2)} \phi\left(g_{B}\right) \quad \text { where } \quad \phi\left(g_{B}\right)=-\frac{1}{2} \ln \operatorname{det} R\left(g_{B}\right) .
$$

The same kind of expression has already been encountered in the investigation of the GL(1|1) WZW model, cf. 31, 32, 36. It is obvious that $\phi$ vanishes whenever $\mathfrak{g}_{0}$ is a semisimple Lie algebra. A non-trivial dilaton is, however, a feature of the series $\operatorname{osp}(2 \mid 2 n), \operatorname{sl}(m \mid n)$ and $\operatorname{gl}(m \mid n)$ or, in other words, of most basic Lie superalgebras of type I. The precise reason for the claimed form of renormalization, i.e. the modification of the metric and the appearance of the dilaton, will become clear in the following section when we discuss the full quantum symmetry of the supergroup WZW model. At the moment let us just restrict ourselves to the comment that the dilaton is required in order to ensure the supergroup invariance of the path integral measure for the free fermion realization, i.e. the description of the WZW model in terms of the Lagrangian (3.9).

Before we conclude this subsection, let us quickly return to the fermionic terms of the Lagrangian (3.9) which may be rewritten in an even more explicit form using

$$
g_{B} \bar{p} g_{B}^{-1}=g_{B} S_{2 b} \bar{p}^{b} g_{B}^{-1}=S_{2 a} R_{b}^{a}\left(g_{B}\right) \bar{p}^{b} .
$$

The result for the interaction term is

$$
\mathcal{S}_{\text {int }}\left[g_{B}, p, \bar{p}\right]=-\frac{i}{2 \pi} \int p_{a} R_{b}^{a}\left(g_{B}\right) \bar{p}^{b} d z \wedge d \bar{z}
$$

In an operator formulation, the object $R^{a}{ }_{b}\left(g_{B}\right)$ should be interpreted as a vertex operator of the bosonic WZW model, transforming in the representation $R \otimes R^{*}$. We may consider the interaction term $p_{a} R_{b}^{a}\left(g_{B}\right) \bar{p}^{b}$ as a screening current. The term (3.13) enters the perturbative expansion of correlation functions when starting from the decoupled theory of bosons and fermions. We would like to stress that the series truncates at finite order for any given correlator since $p$ and $\bar{p}$ are fermionic fields.

Note that the screening current entering (3.13) is non-chiral by definition, a feature that is not really specific to supergroups but applies equally well to free field resolutions of bosonic models. Nevertheless, the existing literature on free field constructions did not pay much attention to this point. Actually, the distinction is not really relevant for purely bosonic WZW models because of their simple factorization into left and right movers. In the present context, however, a complete non-chiral treatment must be enforced in order to capture and understand the special properties of supergroup WZW models.

\subsection{Algebraic treatment}

While our previous considerations took place entirely on a Lagrangian level, they also have a purely algebraic counterpart. In this section we will show, how the current algebra $\hat{\mathfrak{g}}$ can be embedded into a theory described by the current algebra $\mathfrak{g}_{\underline{0}}$ and free fermions. This embedding will lead to a natural class of representations and, eventually, to a natural modular invariant partition function.

\subsubsection{Current algebra}

In what follows we shall focus on the holomorphic sector of the theory. Our first goal is to rephrase the current algebra (3.2) in terms of the special basis chosen in Section 3.2. In the bosonic subsector we find

$$
K^{i}(z) K^{j}(w)=\frac{\kappa^{i j}}{(z-w)^{2}}+\frac{i f^{i j}{ }_{l} K^{l}(w)}{z-w} .
$$


The transformation properties of the fermionic currents are

$$
K^{i}(z) S_{1}^{a}(w)=-\frac{\left(R^{i}\right)^{a}{ }_{b} S_{1}^{b}(w)}{z-w} \quad \text { and } \quad K^{i}(z) S_{2 a}(w)=\frac{S_{2 b}(w)\left(R^{i}\right)^{b}{ }_{a}}{z-w}
$$

Finally we need to specify the operator product of the fermionic currents,

$$
S_{1}^{a}(z) S_{2 b}(w)=\frac{\delta_{b}^{a}}{(z-w)^{2}}-\frac{\left(R^{i}\right)_{b}^{a} \kappa_{i j} K^{j}(w)}{z-w}
$$

The previous operator product expansions (OPEs) are straightforward adaptions of the commutation relations (3.4). The central extension is determined by the invariant metric $\langle\cdot, \cdot\rangle$.

The current superalgebra above defines a chiral vertex algebra via the Sugawara construction. As usual, the corresponding energy momentum tensor is obtained by contracting the currents with the inverse of a distinguished invariant and non-degenerate metric. The appropriate fully renormalized (hence the subscript "full-ren") metric is defined by

$$
\begin{aligned}
\left\langle K^{i}, K^{j}\right\rangle_{\text {full-ren }} & =\left(\Omega^{-1}\right)^{i j}=\kappa^{i j}-\gamma^{i j}-\frac{1}{2} f_{n}^{i m} f_{m}^{j n}, \\
\left\langle S_{1}^{a}, S_{2 b}\right\rangle_{\text {full-ren }} & =\left(\Omega^{-1}\right)^{a}{ }_{b}=\delta_{b}^{a}+\left(R^{i} \kappa_{i j} R^{j}\right)^{a}{ }_{b}
\end{aligned}
$$

and it is the result of adding half the Killing form of the Lie superalgebra $\mathfrak{g}$ to the original classical metric $\langle\cdot, \cdot\rangle$ Note that some of the terms in the fully renormalized metric (3.17) can be identified with the (partially) renormalized metric (3.10) which we introduced when deriving the free fermion Lagrangian. The energy momentum tensor of our theory involves the inverse of the fully renormalized metric,

$$
T=\frac{1}{2}\left[K^{i} \Omega_{i j} K^{j}-S_{1}^{b} \Omega_{b}^{a} S_{2 a}+S_{2 a} \Omega_{b}^{a} S_{1}^{b}\right]
$$

Both, currents and energy momentum tensor, may similarly be defined for the antiholomorphic sector. The appearance of a renormalized metric in the Sugawara construction is a common feature. Supergroup WZW models are certainly not exceptional in this respect.

\subsubsection{Free fermion realization}

Our next aim is to describe the current superalgebra defined above and the associated primary fields in terms of the decoupled system of bosons and fermions that appear in the Lagrangian (3.9). As one of our ingredients we shall employ the bosonic current algebra

$$
K_{B}^{i}(z) K_{B}^{j}(w)=\frac{(\kappa-\gamma)^{i j}}{(z-w)^{2}}+\frac{i f^{i j}{ }_{l} K_{B}^{l}(w)}{z-w}
$$

which is defined using the (partially) renormalized metric $\langle\cdot, \cdot\rangle_{\text {ren }}$ which has been introduced in eq. (3.10). In addition, we need $r$ free fermionic ghost systems, each of central charge $c=-2$. They are described by fields $p_{a}(z)$ and $\theta^{a}(z)$ with OPE

$$
p_{a}(z) \theta^{b}(w)=\frac{\delta_{a}^{b}}{z-w}
$$

and spins $h=1$ and $h=0$, respectively. The fermionic fields are assumed to have trivial operator product expansions with the bosonic generators. By construction, the currents $K_{B}^{i}$ and the fields $p_{a}, \theta^{b}$ generate the chiral symmetry of the field theory whose action is

$$
\mathcal{S}_{0}\left[g_{B}, p, \theta\right]=\mathcal{S}_{\text {ren }}^{\mathrm{WZW}}\left[g_{B}\right]+\mathcal{S}_{\text {free }}[\theta, \bar{\theta}, p, \bar{p}] .
$$

Our full WZW theory may be considered as a deformation of this theory, once we take into account the interaction term between bosons and fermions, see eq. (3.13).

\footnotetext{
${ }^{16}$ Again, this renormalization does not need to be multiplicative.
} 
It is easy to see that the decoupled action (3.21) defines a conformal field theory with energy momentum tensor

$$
T_{0}=\frac{1}{2}\left[K_{B}^{i} \Omega_{i j} K_{B}^{j}+\operatorname{tr}\left(\Omega R^{i}\right) \kappa_{i j} \partial K_{B}^{j}\right]-p_{a} \partial \theta^{a}
$$

Note the existence of the dilaton contributions, i.e. terms linear in derivatives of the currents. In addition to the conformal symmetries, the action (3.21) is also invariant under a $\hat{\mathfrak{g}} \oplus \hat{\mathfrak{g}}$ current superalgebra. The corresponding holomorphic currents are defined by the relations (normal ordering is implied)

$$
\begin{aligned}
K^{i}(z) & =K_{B}^{i}(z)+p_{a}\left(R^{i}\right)^{a}{ }_{b} \theta^{b}(z) \\
S_{1}^{a}(z) & =\partial \theta^{a}(z)+\left(R^{i}\right)^{a}{ }_{b} \kappa_{i j} \theta^{b} K_{B}^{j}(z)-\frac{1}{2}\left(R^{i}\right)^{a}{ }_{c} \kappa_{i j}\left(R^{j}\right)^{b}{ }_{d} p_{b} \theta^{c} \theta^{d}(z) \\
S_{2 a}(z) & =-p_{a}(z) .
\end{aligned}
$$

It is a straightforward exercise, even though slightly cumbersome and lengthy, to check that this set of generators reproduces the operator product expansions (3.14), (3.15) and (3.16). Obviously, a similar set of currents may be obtained for the antiholomorphic sector. Given the representation (3.23) for the current superalgebra one may also check the equivalence of the expressions (3.18) and (3.22) for the energy momentum tensors.

\subsubsection{Chiral representation theory}

The representation theory of the bosonic current algebra $\hat{\mathfrak{g}}_{0}$ and of the fermions $p \theta$ is well understood (see, e.g., [2, 28, and references therein). It will thus not come as a surprise that the free fermion realization outlined in the previous section provides a natural route to the construction of representations of $\hat{\mathfrak{g}}$. In a sense, the conformal embedding

$$
(\hat{\mathfrak{g}},\langle\cdot, \cdot\rangle) \hookrightarrow \mathcal{U}\left(\left(\hat{\mathfrak{g}}_{\underline{0}},\langle\cdot, \cdot\rangle_{\text {ren }}\right) \oplus\left\{p_{a}, \theta^{a}\right\}\right)
$$

constructed in eq. (3.23) is to the current superalgebra $\hat{\mathfrak{g}}$ what the distinguished $\mathbb{Z}$-grading (2.14) was to the underlying horizontal subalgebra $\mathfrak{g}$ - with the slight complication that the metric needs to be renormalized 17

In order to substantiate our claim, let us now define the current superalgebra analog of Kac modules. The starting point is a simple module $\hat{L}_{\lambda}$ of $\left(\hat{\mathfrak{g}}_{\underline{0}},\langle\cdot, \cdot\rangle_{\text {ren }}\right)$ and the vacuum module $\hat{\Lambda}$ of the free ghosts $\left\{p_{a}, \theta^{a}\right\}$. The module $\hat{\Lambda}$ is defined by the highest weight conditions

$$
p_{n}^{a}|0\rangle=\theta_{n+1}^{a}|0\rangle=0 \quad \text { for } n \geq 0
$$

and it is the unique irreducible module, assuming integer moding of the fermions 18 The current superalgebra $(\hat{\mathfrak{g}},\langle\cdot, \cdot\rangle)$ is then acting in the tensor product

$$
\hat{\mathcal{K}}_{\lambda}=\hat{L}_{\lambda} \otimes \hat{\Lambda}
$$

It can be shown 38 that this action is typically irreducible (i.e. $\hat{\mathcal{K}}_{\lambda}=\hat{\mathcal{L}}_{\lambda}$ ) and that the conformal weight of the associated field is described by the (renormalized) Casimir eigenvalues

$$
h_{\lambda}=\frac{1}{2} C_{\lambda}^{\text {full-ren }} .
$$

The corresponding Casimir operator is given by $C^{\text {full-ren }}=K^{i} \Omega_{i j} K^{j}+\operatorname{tr}\left(\Omega R^{i}\right) \kappa_{i j} K^{j}$ and agrees with the zero-mode contribution to eq. (3.22). In cases where $\hat{\mathcal{K}}_{\lambda}$ contains singular vectors (necessarily fermionic), it will be called atypical. In such a case, one can define the irreducible representation $\hat{\mathcal{L}}_{\lambda}$ as the quotient of $\hat{\mathcal{K}}_{\lambda}$ by its maximal ideal $\hat{\mathcal{N}}_{\lambda}$. It is the power of our free fermion realization that it automatically takes care of all bosonic null vectors while ignoring the fermionic ones (if present).

\footnotetext{
${ }^{17}$ In more abstract terms, the renormalization is likely to correspond to the shift with the Weyl vector.

${ }^{18}$ Other modings are obtained by spectral flow, see Section 3.3 .4
} 
Let us dwell a bit on the structure of $\hat{\mathcal{K}}_{\lambda}$. First of all, it is obvious that the space of ground states of $\hat{\mathcal{K}}_{\lambda}$ coincides with $\mathcal{K}_{\lambda}$ since $\hat{L}_{\lambda}$ is built on top of the $\mathfrak{g}_{\underline{0}}$-module $L_{\lambda}$ and $\hat{\Lambda}$ on top of $\Lambda\left(\mathfrak{g}_{-1}\right)$, compare eq. (2.18). In particular, $\hat{\mathcal{K}}_{\lambda}$ will certainly be atypical (i.e. contain fermionic singular vectors) if $\mathcal{K}_{\lambda}$ is atypical. On the other hand, $\hat{\mathcal{K}}_{\lambda}$ can also be atypical if $\mathcal{K}_{\lambda}=\mathcal{L}_{\lambda}$ is typical. In that case, the module contains fermionic singular vectors which are located at higher energy levels. In all examples we are aware of, the corresponding modules $\hat{\mathcal{K}}_{\lambda}$ arise as the spectral flow image of a different atypical module $\hat{\mathcal{K}}_{\mu}$ that has its singular vectors on the ground state level. We believe that this is true in general.

We finally note that the character of the Kac modules $\hat{\mathcal{K}}_{\lambda}$ follows immediately from the known characters of the constituents in eq. (3.26) and modular transformations are easily worked out. The story becomes more subtle if one is interested in characters of atypical irreducible modules $\hat{\mathcal{L}}_{\lambda}$. As far as we know, a general theory has not been established in that case despite of the existence of certain results and conjectures in the mathematical literature [46, 86, 87, 88, 89, 90, It is nevertheless worth pointing out that there appear to be interesting connections to Mock modular forms [91, 92, 93. For the low-rank examples of $\operatorname{gl}(1 \mid 1), \operatorname{sl}(2 \mid 1)$ and $\operatorname{psl}(2 \mid 2)$ rather explicit expressions for characters and (partially) modular transformations have been derived in the physics literature [32, 15, 24, 37, 94. Fortunately, knowledge about the characters of atypical representations is not required to prove modular invariance for the partition function of (type I) supergroup WZW models. This will be shown in Section 3.3.5.

\subsubsection{Spectral flow automorphisms}

In the previous subsection we have silently skipped over one rather important element in the representation theory of current (super)algebras: The spectral flow automorphisms. As we shall recall momentarily, spectral flow automorphisms describe symmetry transformations in the representation theory of current algebras. Furthermore, they seem to be realized as exact symmetries of the WZW models on supergroups, a property that makes them highly relevant for the discussion of partition functions.

Throughout the following discussion, we shall denote (spectral flow) automorphisms of the current superalgebra $\hat{\mathfrak{g}}$ by $\omega$. We shall mostly assume that the action of $\omega$ is consistent with the boundary conditions for currents, i.e. that it preserves the integer moding of the currents. In the context of representation theory, any such spectral flow automorphism $\omega$ defines a map on the set of (isomorphism classes of) representations $\rho: \hat{\mathfrak{g}} \rightarrow \operatorname{End}(\hat{\mathcal{M}})$ via concatenation, $\omega(\rho)=\rho \circ \omega:$ $\hat{\mathfrak{g}} \rightarrow \operatorname{End}(\hat{\mathcal{M}})$. Since the automorphism shuffles the modes of the currents, it usually changes the energy level of states. It is also well known that spectral flow may yield representations whose energy spectrum is not bounded from below. Such representations are relevant for the study of WZW models on non-compact (super)groups such as $\operatorname{SL}(2, \mathbb{R})$ or $\operatorname{PSU}(1,1 \mid 2)$ [95, 24.

In line with our general strategy, we would like to establish that spectral flow automorphisms $\omega$ of the current superalgebra are uniquely determined by their action on the bosonic generators. A spectral flow automorphism $\omega: \hat{\mathfrak{g}}_{\underline{0}} \rightarrow \hat{\mathfrak{g}}_{\underline{0}}$ of the bosonic subalgebra $\hat{\mathfrak{g}}_{\underline{0}}$ is, by definition, a linear map

$$
\omega\left(K^{i}(z)\right)=\left(W_{\underline{0}}\right)^{i}{ }_{j}(z) K^{j}(z)+w_{\underline{0}}^{i} z^{-1}
$$

which is consistent with the OPE (3.14). The map $W_{\underline{0}}(z)=z^{\zeta_{0}}$ is defined in terms of an endomorphism $\zeta_{\underline{0}}: \mathfrak{g}_{\underline{0}} \rightarrow \mathfrak{g}_{\underline{0}}$ of the horizontal subalgebra. While the eigenvalues of $\zeta_{\underline{0}}$ determine how the spectral flow shifts the modes of the currents, the vector $w_{0}^{i}$ affects only the zero-modes. In order to preserve the trivial monodromy under rotations around the origin we will assume that $W_{\underline{0}}(z)$ is a meromorphic function, i.e. that all the eigenvalues of $\zeta_{\underline{0}}$ are integer. Inserting the transformation (3.28) into the operator product expansions (3.14) leaves one with the relation

$$
\left(\zeta_{\underline{0}}\right)_{j}^{i}=f^{i k}{ }_{l} \kappa_{k j} w_{\underline{0}}^{l}
$$

while all other constraints are satisfied automatically. Hence the only free parameter is the shift vector $w_{\underline{0}}^{i}$. In the case of a semisimple Lie algebra $\mathfrak{g}_{\underline{0}}$ (which leads to a non-degenerate Killing form) this argument can also be reversed and hence it allows to express $w_{\underline{0}}^{i}$ in terms of $\zeta_{\underline{0}}$. Any vector $w_{\underline{0}}^{i}$ which leads to a matrix $\zeta_{\underline{0}}$ with integer eigenvalues under the identification (3.29) will accordingly be referred to as a spectral flow automorphism of $\hat{\mathfrak{g}}_{\underline{0}}$ from now on. 
Given the insights of the previous paragraphs it is now fairly straightforward to extend the spectral flow automorphism $\omega: \hat{\mathfrak{g}}_{\underline{0}} \rightarrow \hat{\mathfrak{g}}_{\underline{0}}$ to the full current superalgebra. To this end, we introduce the element

$$
\zeta_{\underline{1}}=-R^{i} \kappa_{i j} w_{\underline{0}}^{j} .
$$

Following the discussion in the bosonic sector, we now introduce a function $W_{1}(z)=z^{\zeta_{1}}$. This allows us to define the action of the spectral flow automorphism $\omega$ on the fermionic currents by

$$
\omega\left(S_{1}^{a}(z)\right)=\left(W_{1}\right)^{a}{ }_{b}(z) S_{1}^{b}(z) \quad, \quad \omega\left(S_{2 a}(z)\right)=S_{1 b}(z)\left(\bar{W}_{1}\right)^{b}{ }_{a}(z)
$$

where $\bar{W}_{\underline{1}}$ denotes the inverse of $W_{\underline{1}}$.

We would also like to argue that the spectral flow symmetry is consistent with the free fermion representation (3.23). To be more specific, we shall construct an automorphism on the chiral algebra of the decoupled system generated by the currents $K_{B}^{i}(z)$ and the free ghosts $p_{a}(z)$ and $\theta^{a}(z)$ that reduces to the expressions above if we plug the transformed fields into the defining equations (3.23). In this context the most important issue is to understand how the renormalization of the metric $\kappa \rightarrow \kappa-\gamma$ affects the action of the spectral flow. As it turns out, the data $\zeta_{0}$ which gave rise to a spectral flow automorphism of $\hat{\mathfrak{g}}_{\underline{0}}$ above, can also be used to define a spectral flow automorphism of the renormalized current algebra, i.e. of the algebra that is generated by $K_{B}^{j}$ with operator products given in eq. (3.19). Only the shift vector $w_{\underline{0}}^{i}$ of the zero modes needs a small adjustment such that the new spectral flow action reads

$$
\omega\left(K_{B}^{i}(z)\right)=\left(W_{\underline{0}}\right)^{i}{ }_{j}(z) K_{B}^{j}(z)+w_{B}^{i} z^{-1} \quad \text { where } \quad w_{B}^{i}=w_{\underline{0}}^{i}+\operatorname{tr}\left(\zeta_{\underline{1}} R^{i}\right)
$$

Note that $\zeta_{0}$ is not changed and hence it has the same (integer) eigenvalues as before. In order to obtain an automorphism which is compatible with the free field construction we finally need to introduce the transformations

$$
\omega\left(p_{a}(z)\right)=p_{b}(z)\left(\bar{W}_{\underline{1}}\right)_{a}^{b}(z) \quad, \quad \omega\left(\theta^{a}(z)\right)=\left(W_{\underline{1}}\right)_{b}^{a}(z) \theta^{b}(z) .
$$

It is then straightforward but lengthy to check that the previous transformations define an automorphism of the algebra generated by $p_{a}, \theta^{a}$ and $K_{B}^{j}$ that descends to the original spectral flow automorphism $\omega$ of our current superalgebra $\hat{\mathfrak{g}}$. During the calculation one has to be aware of normal ordering issues.

In conclusion we have shown that any spectral flow automorphism of the bosonic subalgebra of a current superalgebra (related to a Lie superalgebra of type I) can be extended to the full current superalgebra. Furthermore, this extension was seen to be consistent with our free fermion realization. Let us remark that even if we start with a spectral flow automorphism $\omega$ preserving periodic boundary conditions for bosonic currents, the lifted spectral flow $\omega$ does not necessarily have the same property on fermionic generators. Only those spectral flow automorphisms $\omega: \hat{\mathfrak{g}} \rightarrow \hat{\mathfrak{g}}$ for which $W_{\underline{1}}$ is meromorphic as well seem to arise as symmetries of WZW models on supergroups. Nevertheless, also non-meromorphic spectral flows turn out to be of physical relevance. They can be used to describe the twisted sectors of orbifold theories.

\subsubsection{The partition function: Decoupled versus interacting theory}

We now return to the important question of characterizing the bulk state space of the WZW model on the supergroup $\mathrm{G}$. With regard to the decoupled action $\mathcal{S}_{0}$ from eq. (3.21) it is clear that the partition function factorizes into the partition function of the (renormalized) bosonic WZW model on $\mathrm{G}_{\underline{0}}$ (including spectral flow sectors if necessary) and the free ghosts

$$
Z(G,\langle\cdot, \cdot\rangle)=Z\left(G_{\underline{0}},\langle\cdot, \cdot\rangle_{\mathrm{ren}}\right) \cdot Z\left(p_{a}, \theta^{a}\right)
$$

see 38 for a more detailed discussion. In this form, modular invariance is manifest. What is much less obvious: The partition function remains the same if the interaction term $\mathcal{S}_{\text {int }}$ in eq. (3.13) is switched on which converts the decoupled theory into the supergroup WZW model.

In order to understand the logic of the previous statement we have to resort to our results on the harmonic analysis on supergroups in Section 2.4. Following the standard reasoning [96, the 
space of ground states of a WZW model can be described by the algebra of functions $\mathcal{F}(\mathrm{G})$ in the limit of large volume. The action of the current algebra on these states reduces to the biregular action of $\mathrm{G}$ on $\mathcal{F}(\mathrm{G})$ and the energy is approximated by the eigenvalue of the Laplacian which, in turn, corresponds to half of the (unrenormalized) quadratic Casimir operator. It may be shown that that the Lie derivatives for the left regular action are given by 38]

$$
\begin{aligned}
& K^{i}=K_{B}^{i}-\left(R^{i}\right)^{a}{ }_{b} \theta^{b} \partial_{a} \quad S_{2 a}=-\partial_{a} \\
& S_{1}^{a}=R_{b}^{a}\left(g_{B}\right) \bar{\partial}^{b}+\left(R^{i}\right)^{a}{ }_{b} \theta^{b} \kappa_{i j} K_{B}^{j}-\frac{1}{2}\left(R^{i}\right)^{a}{ }_{c} \kappa_{i j}\left(R^{j}\right)^{b}{ }_{d} \theta^{c} \theta^{d} \partial_{b},
\end{aligned}
$$

As we see, this does not quite correspond to the zero mode action of the currents (3.23) due to the extra contribution in $S_{1}^{a}$ which involves $\bar{\partial}^{b}=\partial / \partial \bar{\theta}_{a}$. In view of this issue it is tempting to simply drop the troublesome term. Even though that might seem a rather arbitrary modification at first, it turns out that the corresponding truncated differential operators $\mathbb{K}^{i}=K^{i}, \mathbb{S}_{2 a}=S_{2 a}$ and

$$
\mathbb{S}_{1}^{a}=\left(R^{i}\right)_{b}^{a} \theta^{b} \kappa_{i j} K_{B}^{j}-\frac{1}{2}\left(R^{i}\right)_{c}^{a} \kappa_{i j}\left(R^{j}\right)^{b}{ }_{d} \theta^{c} \theta^{d} \partial_{b}
$$

also satisfy the commutation relations of $\mathfrak{g}$. These considerations extend to the full biregular action of $\mathfrak{g} \oplus \mathfrak{g}$.

We thus conclude that $\mathcal{F}(\mathrm{G})$ admits two different actions of $\mathfrak{g} \oplus \mathfrak{g}$. The action of the generators $\left(K^{i}, S_{1}^{a}, S_{2 a}\right)$ and their barred analogues leads to the decomposition (2.34) involving the non-chiral representations $\mathcal{I}_{[\sigma]}$. On the other hand, the action of the generators $\left(\mathbb{K}^{i}, \mathbb{S}_{1}^{a}, \mathbb{S}_{2 a}\right)$ leads to a different decomposition of $\mathcal{F}(\mathrm{G})$ in terms of sums over products $\mathcal{K}_{\lambda} \otimes \mathcal{K}_{\lambda}^{*}$. We shall denote the corresponding module by $\mathbb{F}(\mathrm{G})$. The modules $\mathcal{F}(\mathrm{G})$ and $\mathbb{F}(\mathrm{G})$ both have the same character since they are isomorphic as $\mathfrak{g}_{\underline{0}} \oplus \mathfrak{g}_{\underline{0}}$-modules [38]. On the other hand, the Laplace operator can be diagonalized on $\mathbb{F}(\mathrm{G})$ while it is not diagonalizable on $\mathcal{F}(\mathrm{G})$. From a physical perspective, $\mathbb{F}(\mathrm{G})$ corresponds to the space of ground states of the decoupled theory while $\mathcal{F}(\mathrm{G})$ is associated to the full WZW model. Our previous comments imply the equality of the respective partition functions and the occurrence of logarithmic correlation functions in the supergroup WZW model. A more detailed discussion of these issues can be found in 38. For completeness we should mention that WZW models for non-simply connected supergroups can be described by means of an orbifolding procedure which is implemented in terms of simple currents (e.g. spectral flow automorphisms).

It is instructive to contrast the previous statements with the logic of ordinary free field resolutions [97, 98. In that case, the role of $\left(\hat{\mathfrak{g}}_{0},\langle\cdot, \cdot\rangle\right)$ is played by free scalar fields (associated with the Cartan subalgebra $\mathfrak{h}$ ) and the root generators give rise to either free bosonic or fermionic ghost systems, depending on the nature of the roots. In that case, the total partition function is not the product of the individual partition functions. Rather, one needs to pass over to a cohomology which is defined in terms of screening currents. This removes the singular vectors from the state space. In contrast, the fermionic singular vectors arising from the free fermion realization of supergroup WZW models need not be removed and the partition functions of the interacting and the non-interacting theory agree. Nevertheless there are still differences on the level of the state space. While the non-interacting theory is free of logarithms and exhibits a trivial holomorphic factorization, the interacting theory is logarithmic and has a non-chiral state space.

\subsection{Free field realization the $\operatorname{OSP}(m \mid 2 n)$ WZW model at level $k=1$}

The distinguished $\mathbb{Z}$-grading (2.14) of type II Lie superalgebras permits to mimic the Gauss decomposition of Section 3.2. However, in practice this turns out not to be of particular use since it artificially divides the simple $\mathfrak{g}_{0}$-module $\mathfrak{g}_{1}$ into two parts $\mathfrak{g}_{ \pm 1}$ and since the corresponding fermions cease to be nilpotent. Instead, following [39], we wish to concentrate here on a true free field construction for the OSP $(m \mid 2 n)$ WZW model which, however, has the drawback that it only works for level $k=1$. On the other hand, this model features in some of the most interesting applications with orthosymplectic supersymmetry [99, 100, 39, 101. 


\subsubsection{Current algebra}

The basic fields which enter the construction are a set of $m$ free fermions $\psi$ and $n$ bosonic $\beta \gamma$ systems with OPE

$$
\psi^{i}(z) \psi^{j}(w)=\frac{\delta^{i j}}{z-w}, \quad \beta_{a}(z) \gamma^{b}(w)=\frac{\delta_{a}^{b}}{z-w} .
$$

These fields all have conformal dimension $h=1 / 2$ which is guaranteed by choosing the energy momentum tensor

$$
T(z)=-\frac{1}{2} \psi_{i} \partial \psi^{i}(z)+\frac{1}{2}\left[\beta_{a} \partial \gamma^{a}(z)-\gamma^{a} \partial \beta_{a}(z)\right] .
$$

The central charge of the system is easily seen to take the value $c=\frac{m}{2}-n$. In the next step, we will verify that the bilinears in the free fields realize the current superalgebra $\widehat{\mathrm{osp}}(m \mid 2 n)$ at level $k=1$ (with positive level for the orthogonal part and negative level for the symplectic part). In Section 3.4 .2 we will furthermore establish that the corresponding WZW model is equivalent to a suitable orbifold of the free field theory.

In order to construct the currents of $\widehat{\operatorname{osp}}(m \mid 2 n)$ we choose a matrix $X \in \operatorname{osp}(m \mid 2 n)$ and decompose it into blocks according to

$$
X=\left(\begin{array}{c|cc}
\mathcal{E} & \overline{\mathcal{T}} & \mathcal{T} \\
\hline-\mathcal{T}^{t} & \mathcal{F} & \mathcal{G} \\
\overline{\mathcal{T}}^{t} & \overline{\mathcal{G}} & -\mathcal{F}^{t}
\end{array}\right)
$$

where $\mathcal{E}$ is antisymmetric and $\mathcal{G}, \overline{\mathcal{G}}$ are symmetric. A basis for the individual constituents of $X$ is provided by the vectors

$$
\begin{aligned}
& \mathcal{E}_{i j}=e_{i j}-e_{j i} \quad(i<j), \quad \mathcal{G}_{a b}=\overline{\mathcal{G}}_{a b}=e_{a b}+e_{b a} \\
& \mathcal{F}_{a b}=e_{a b} \quad \mathcal{T}_{i a}=\overline{\mathcal{T}}_{i a}=e_{i a},
\end{aligned}
$$

where the matrices $e_{\alpha \beta}$ have a single unit entry in row $\alpha$ and column $\beta$. We agree to denote by $E_{i j}$ the supermatrix of the form (3.39) where $\mathcal{E}$ is given by $\mathcal{E}_{i j}$ and all other blocks vanish. The basis elements $F_{a b}, G_{a b}, \bar{G}_{a b}, T_{i a}, \bar{T}_{i a}$ are defined similarly.

In the next step we define a metric on $\operatorname{osp}(m \mid 2 n)$ using the assignment $\langle X, Y\rangle=\frac{1}{2} \operatorname{str}(X Y)$. Evaluating it on the basis elements, the metric is fully specified by

$$
\begin{aligned}
\left\langle E_{i j}, E_{k l}\right\rangle & =-\delta_{i k} \delta_{j l} \quad(\text { for } i<j \text { and } k<l), & & \left\langle F_{a b}, F_{c d}\right\rangle=-\delta_{a d} \delta_{b c} \\
\left\langle G_{a b}, \bar{G}_{c d}\right\rangle & =-\delta_{a c} \delta_{b d} \quad(\text { for } a \neq b \text { and } c \neq d), & & \left\langle G_{a a}, \bar{G}_{b b}\right\rangle=-2 \delta_{a b} \\
\left\langle T_{i a}, \bar{T}_{j b}\right\rangle & =\delta_{i j} \delta_{a b} . & &
\end{aligned}
$$

The free field representation of $\widehat{\mathrm{osp}}(m \mid 2 n)$ is defined in terms of the assignments

$$
\begin{aligned}
E_{i j}(z) & =\left(\psi_{i} \psi_{j}\right)(z), & & F_{a b}(z)=-\left(\beta_{a} \gamma_{b}\right)(z) \\
G_{a b}(z) & =\left(\beta_{a} \beta_{b}\right)(z), & & \bar{G}_{a b}(z)=-\left(\gamma_{a} \gamma_{b}\right)(z) \\
T_{i a}(z) & =i\left(\psi_{i} \beta_{a}\right)(z), & & \bar{T}_{i a}(z)=-i\left(\psi_{i} \gamma_{a}\right)(z) .
\end{aligned}
$$

The OPEs between these currents present the proper affinization of the commutation relations of the corresponding matrices defined in (3.40), with a central extension specified by the metric (3.42) (this is equivalent to having level $k=1$ ). Assuming $m \neq 2 n+1$, the energy momentum tensor can then be defined in the standard Sugawara form

$$
\begin{aligned}
T(z)=\frac{\left(J^{\mu} J_{\mu}\right)(z)}{2\left(k+g^{\vee}\right)}= & \frac{1}{2\left(k+g^{\vee}\right)}\left[-\sum_{i<j=1}^{m}\left(E_{i j}^{2}\right)-\sum_{a, b=1}^{n}\left(F_{a b} F_{b a}\right)-\sum_{a<b=1}^{n}\left(\left\{G_{a b}, \bar{G}_{a b}\right\}\right)\right. \\
& \left.-\frac{1}{2} \sum_{a=1}^{n}\left(\left\{G_{a a}, \bar{G}_{a a}\right\}\right)-\sum_{i=1}^{m} \sum_{a=1}^{n}\left(\left[T_{i a}, \bar{T}_{i a}\right]\right)\right] .
\end{aligned}
$$


Here, the dual Coxeter number is given by $g^{\vee}=m-2 n-219$ It may be checked that $T(z)$ reduces to the energy momentum tensor (3.38) after using the replacements (3.43).

\subsubsection{Comments on the state space}

The OSP $(m \mid 2 n)$ WZW model can be realized as an orbifold of the free field theory generated by the fields $\psi^{i}$ and $\beta_{a}, \gamma^{b}$. The orbifold guarantees that all free fields obey the same boundary conditions, i.e. they are either all periodic or all anti-periodic. This in turn ensures that the currents defined in (3.43) - which are bilinears in the free fields - are all periodic. However, before we discuss the implementation of the orbifold, we first focus on the chiral representation theory of a single fermion or $\beta \gamma$-system, respectively.

For a single Majorana fermion $\psi$ we need two distinct representations, one for periodic boundary conditions (half-integer moding, $\nu=0$ ) and one for anti-periodic boundary conditions (integer moding, $\nu=1 / 2$ ). The associated ground states $|\nu\rangle$ are defined by the highest weight conditions

$$
\psi_{n}|\nu\rangle=0 \text { for } n>0 \text {. }
$$

It is well-known (see, e.g., 42]) that the two representations just constructed are not irreducible over the Virasoro algebra. The corresponding characters rather decompose as

$$
\chi_{\psi}^{(0)}(q)=\chi_{0}(q)+\chi_{1 / 2}(q) \quad \text { and } \quad \chi_{\psi}^{(1 / 2)}(q)=2 \chi_{\sigma}(q)
$$

into the characters of the Ising model. The field $1 / 2$ is the unique simple current of the latter, with fusion rules $1 / 2 \otimes 1 / 2=0$ and $1 / 2 \otimes \sigma=\sigma$.

The situation is slightly different for the bosonic $\beta \gamma$-system [102]. As before, we shall consider sectors which differ by the choice of boundary conditions and we introduce a family of ground states $|\nu\rangle$ for $\nu \in \frac{1}{2} \mathbb{Z}$. These states are characterized by the conditions

$$
\beta_{r+\nu}|\nu\rangle=0 \quad, \quad \gamma_{r-\nu}|\nu\rangle=0 \quad \text { for } \quad r=1 / 2,3 / 2,5 / 2, \ldots
$$

From the ground states we generate the corresponding sectors by application of raising operators. If we assign charges $q_{\beta}=1 / 2$ and $q_{\gamma}=-1 / 2$ to the modes of the fields $\beta$ and $\gamma$, respectively, and $q_{\nu}=\nu / 2$ to the ground state $|\nu\rangle$ the generating function for the sector $\nu$ reads,

$$
\chi^{(\nu)}(q, y)=q^{\frac{1}{24}-\frac{\nu^{2}}{2}} y^{\frac{\nu}{2}} \prod_{n=0}^{\infty} \frac{1}{\left(1-y^{\frac{1}{2}} q^{n+\frac{1}{2}-\nu}\right)\left(1-y^{-\frac{1}{2}} q^{n+\frac{1}{2}+\nu}\right)}=\frac{q^{-\nu^{2} / 2} y^{\frac{\nu}{2}} \eta(q)}{\theta_{4}\left(q, y^{1 / 2} q^{-\nu}\right)}
$$

All the constructed sectors carry an action of an affine $\widehat{\mathrm{sl}}(2)$ current algebra at level $k=-1 / 2$ 102. In terms of the fields $\beta$ and $\gamma$ the three currents are constructed as follows,

$$
E^{+}(z)=\frac{1}{2} \beta^{2}(z), \quad H(z)=-\frac{1}{2}(\beta \gamma)(z), \quad E^{-}(z)=-\frac{1}{2} \gamma^{2}(z)
$$

Consequently, we can decompose the generating functions (3.48) into characters of irreducible representations of $\widehat{\mathrm{sl}}(2)_{-1 / 2}$. In case of $\chi^{(0)}$, for example, the decomposition is given by

$$
\chi^{(0)}(q, y)=\frac{\eta(q)}{\theta_{4}\left(q, y^{1 / 2}\right)}=\chi_{0}^{k=-1 / 2}(q, y)+\chi_{1 / 2}^{k=-1 / 2}(q, y) .
$$

The two characters on the right hand side belong to irreducible highest weight representations with lowest weight $h=\epsilon \in\{0,1 / 2\}$,

$$
\chi_{\epsilon}^{k=-1 / 2}(q, y)=\frac{\eta(q)}{2}\left[\frac{1}{\theta_{4}\left(q, y^{1 / 2}\right)}+(-1)^{2 \epsilon} \frac{1}{\theta_{3}\left(q, y^{1 / 2}\right)}\right] .
$$

Let us note that the ground states transform in representations of spin $j=\epsilon$. Nevertheless, we shall continue to think of the subscript of $\chi$ as the conformal weight rather than the spin. Decomposition

\footnotetext{
${ }^{19}$ Since we are dealing with the fixed form (3.42) there is a deviation from Table 1 for $m \lesssim 2 n$. The discrepancy is due to different normalization conventions.
} 
formulas similar to (3.50) exist for all the other functions (3.48). All of them are related by the action of spectral flow automorphisms [102]. In particular, we have

$$
\chi^{(1 / 2)}=\chi_{\sigma ;+}^{k=-1 / 2}+\chi_{\sigma ;-}^{k=-1 / 2} \quad \text { with } \chi_{\sigma ; \pm}(q, y)=\frac{y^{1 / 4} \eta(q)}{2}\left[\frac{1}{i \theta_{1}\left(q, y^{-1 / 2}\right)} \pm \frac{1}{\theta_{2}\left(q, y^{-1 / 2}\right)}\right] .
$$

The two characters on the left hand side belong to the two irreducible lowest weight representations of the current algebra with spin $j=1 / 4$ and $j=3 / 4$. Their ground states have the same conformal weight $h=-1 / 8$. In close analogy to the Ising model, the representation with $h=1 / 2$ (and $j=1 / 2)$ is a simple current of the $\widehat{\mathrm{sl}}(2)_{-1 / 2}$ theory [102, 103]. Its action exchanges the two constituents of $\chi^{(\nu)}$.

Restricting our attention to the bosonic subrepresentations, i.e. the representations of the Ising model and of $\widehat{\operatorname{sl}}(2)_{-1 / 2}$, the product of $m$ real fermions and $n \beta \gamma$-systems contains a group $\mathbb{Z}_{2}^{m+n}$ of simple currents that consists of all elements $\eta$ of the form

$$
\eta=\left[\epsilon_{1}, \ldots, \epsilon_{n} ; \epsilon_{n+1}, \ldots, \epsilon_{m+n}\right] \text { with } \epsilon_{i} \in\{0,1 / 2\} .
$$

The first $n$ entries of $\eta$ denote subsectors of the $\beta \gamma$-system while the remaining ones are representing sectors in the Ising models. Requiring the simple currents to have integer conformal dimension leads to the constraint $\sum_{i=1}^{m+n} \epsilon_{i}=0 \bmod 1$. The elements $\eta$ solving this equation generate the abelian group $\Gamma \cong \mathbb{Z}_{2}^{m+n-1}$.

We are now ready to discuss the bulk modular invariant for the full WZW model. The construction of a simple current orbifold proceeds in several steps. To begin with, we have to list all sectors $a$ of the theory which possess vanishing monodromy charge $Q_{a}(\eta)=h_{a}+h_{\eta}-h_{\eta \times a} \bmod 1$. These are then organized into orbits $\mathcal{O}_{a}$ under the action of the simple current group $\Gamma$. According to the standard construction (see e.g. 104]), each such orbit $\mathcal{O}_{a}$ contributes one term $Z_{a}$ to the partition function of the orbifold model, with a coefficient $|\Gamma| /\left|\mathcal{O}_{a}\right|$ that is given by the ratio between the order $|\Gamma|$ of the orbifold group and the length $\left|\mathcal{O}_{a}\right|$ of the orbit. Unfortunately, the characters of the $\widehat{\mathrm{sl}}(2)_{-1 / 2}$ theory exhibit rather peculiar properties such as restricted domains of convergence and relations under spectral flow 105, 106, 107, 108. For this reason, the following result on the bulk state space is based on a rather formal application of the orbifold construction. The full validity of our procedure still remains to be established.

Let us first deal with the sector involving representations with vanishing spectral flow, $\nu=0$. Under the action of $\Gamma$, the sectors with vanishing monodromy charge split into two orbits of maximal length. Hence we are led to the following contribution to the state space,

$$
\mathcal{H}_{0}^{\mathrm{FF}}=\left|\bigoplus_{\eta \in \Gamma} \mathcal{H}_{\eta \times[0, \ldots, 0 ; 0, \ldots, 0]}\right|^{2}+\left|\bigoplus_{\eta \in \Gamma} \mathcal{H}_{\eta \times[0, \ldots, 0 ; 0, \ldots, 0,1 / 2]}\right|^{2}
$$

In this expression we used $|\mathcal{H}|^{2}=\mathcal{H} \otimes \mathcal{H}^{*}$ as an abbreviation in order to mimic the expected expression for the partition function. However, the total theory has to be invariant under the spectral flow symmetry. Hence we have to add twisted contributions $\mathcal{H}_{\nu}^{\mathrm{FF}}$. It was already mentioned above that all the bosonic ghosts and all the fermions have to have identical periodicity conditions in order to not to spoil the $\operatorname{osp}(m \mid 2 n)$ symmetry. Consequently the spectral flow must act diagonally, i.e. simultaneously on all sectors, by half-integer shifts 20 In the fermionic factors, spectral flow by $\nu=1 / 2$ brings us from the $\psi$-module (0) to $(1 / 2)$. Both subrepresentations 0 and $1 / 2$ of $(0)$ are thus mapped to the representation $\sigma$ which, in turn, are fixed under a subgroup $\mathcal{S} \subset \Gamma$ of the group of simple currents. Integer units of the spectral flow, however, do not give anything new since the moding is preserved. In the ghost sectors things works differently because the application of a diagonal spectral flow leads to an infinite number of new representations constructed from the ground states $|\nu\rangle$ for $\nu \in \frac{1}{2} \mathbb{Z}$. Since the orbits of the half-integer spectral flow representations possess a stabilizer subgroup $\mathcal{S}$ of order $|\mathcal{S}|=2^{m-1}$ with respect to the action of $\Gamma$ we finally end

\footnotetext{
${ }^{20}$ It is worth mentioning that these diagonal spectral flow transformations are also the only ones which commute with the action of the orbifold group. Note also that half-integer spectral flow on ghosts and fermions implies integer spectral flow on the currents such as those defined in eq. (3.49) and below.
} 
up with the state space

$$
\begin{aligned}
& \mathcal{H}^{\mathrm{WZW}}=\bigoplus_{\nu \in \frac{1}{2} \mathbb{Z}} \mathcal{H}_{\nu}^{\mathrm{FF}}=\bigoplus_{\nu \in \mathbb{Z}}\left[\left|\bigoplus_{\eta \in \Gamma} \mathcal{H}_{\eta \times[0, \ldots, 0 ; 0, \ldots, 0]}^{(\nu)}\right|^{2}+\left|\bigoplus_{\eta \in \Gamma} \mathcal{H}_{\eta \times[0, \ldots, 0 ; 0, \ldots, 0,1 / 2]}^{(\nu)}\right|^{2}\right] \\
& \oplus 2^{m-1} \bigoplus_{\nu \in \mathbb{Z}+\frac{1}{2}}|\underbrace{\mathcal{H}^{(\nu)} \otimes \cdots \otimes \mathcal{H}^{(\nu)}}_{n \text { factors } \beta \gamma} \otimes \underbrace{\mathcal{H}_{\sigma} \otimes \cdots \otimes \mathcal{H}_{\sigma}}_{m \text { factors Ising }}|^{2} .
\end{aligned}
$$

Our proposal is admittedly somewhat sketchy. In view of the various subtleties related to the $\beta \gamma$ system, the fractional level WZW model $\widehat{\mathrm{sl}}(2)_{-1 / 2}$ and their orbifolds $105,105,106,107,103,108$, it may well be worth revisiting it with more care. Since we will mainly be interested in the boundary theory of the OSP WZW models in this review, we postpone such an analysis to future work.

We note that the example just discussed is one of the rare cases where a supergroup WZW model leads to a theory which may be regarded as compact to some extent since the (negative) level of the affine $\widehat{\mathrm{sp}}(2 n)$ subalgebra is so close to zero that it is pushed into the positive regime by effects of quantum renormalization. Moreover, even though the model as discussed above is presumably not logarithmic due to the restriction to a small level, the theory admits a logarithmic lift by the inclusion of additional zero modes. This is in close analogy to the case of $\widehat{\mathrm{su}}(2)_{-1 / 2}$ [105, 107.

\subsection{Deformations}

Supergroup WZW models may, under certain circumstances, allow for exactly marginal deformations that would usually spoil conformal invariance. These are deformations which either preserve the original global symmetry $\mathrm{G} \times \mathrm{G}$ or some (twisted) diagonal symmetry $\mathrm{G}$. We shall briefly describe the implementation of these deformations in terms of perturbing fields and discuss necessary conditions for their marginality.

\subsection{1 $\mathrm{G} \times \mathrm{G}$ preserving deformations}

WZW models possess an obvious deformation which amounts to allowing for a different normalization of the two contributions to the action (3.1). Since the coefficient of the topological term is quantized (at least for supergroups having a compact simple part) the only freedom is in fact to change the coefficient of the kinetic term. In most cases, especially for all simple bosonic groups, such a deformation would spoil conformal invariance. The only exception occurs if $\mathrm{G}$ is a supergroup with vanishing Killing form as we will now review.

The implementation of the $\mathrm{G} \times \mathrm{G}$-preserving deformation in the operator language is somewhat cumbersome. We have to find a field $\Phi(z, \bar{z})$ that is invariant under $\mathrm{G} \times \mathrm{G}$ and possesses conformal dimension $(h, \bar{h})=(1,1)$. The deformed model would then formally be described by the deformed action $\mathcal{S}_{\text {def }}=\mathcal{S}^{\mathrm{WZW}}+\xi / 2 \pi \int d^{2} z \Phi(z, \bar{z})$. The two currents $J$ and $\bar{J}$ transform non-trivially under either the left or the right action of $\mathrm{G}$ on itself. For this reason, an invariant can only be built by conjugating one of the two currents. In algebraic terms we have to consider the normal ordered operator

$$
\Phi_{\mathrm{G} \times \mathrm{G}}(z, \bar{z})=:\left\langle J(z), \operatorname{Ad}_{g}(\bar{J}(\bar{z}))\right\rangle:=: J^{\mu} \phi_{\mu \nu} \bar{J}^{\nu}:(z, \bar{z})
$$

involving the non-chiral affine primary field $\phi_{\mu \nu}(z, \bar{z})$ which transforms in the representation ad $\otimes$ ad with respect to the symmetry $\mathrm{G} \times \mathrm{G}$. At lowest order in perturbation theory, the field $\Phi_{\mathrm{G} \times \mathrm{G}}(z, \bar{z})$ is marginal if and only if the field $\phi_{\mu \nu}$ has conformal dimensions $(h, \bar{h})=(0,0)$. Since the conformal dimensions of the affine primary field $\phi_{\mu \nu}$ are proportional to the quadratic Casimir in the adjoint representation, this condition is equivalent to the vanishing of the Killing form of G.

It may be shown that the addition of the perturbing field (3.56) to a WZW model leads to a non-chiral deformation of the original current algebra. The structure and physical significance of such current algebras has been discussed in a sequence of papers [109, 110, 111. 
a)

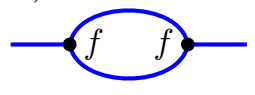

b)

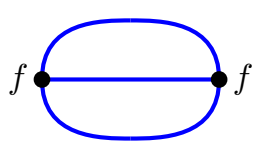

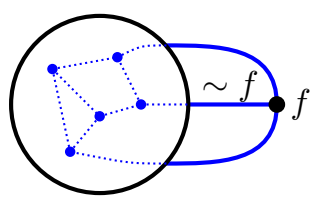

Figure 3: a) The Killing form. b) Vanishing contributions to the $\beta$-function.

\subsubsection{G preserving deformations}

Supergroups with vanishing Killing form admit a second type of deformation. This deformation is easier to describe since it avoids the use of the non-chiral field $\phi_{\mu \nu}(z, \bar{z})$. On the other hand it is more difficult to interpret geometrically since the group structure is broken. In this case the deformation is implemented by the perturbing field

$$
\Phi_{\mathrm{G}}(z, \bar{z})=\langle J(z), \bar{J}(\bar{z})\rangle=J_{\mu} \bar{J}^{\mu}(z, \bar{z}) .
$$

This field transforms non-trivially under the full $\mathrm{G} \times \mathrm{G}$-symmetry but trivially under the diagonal subgroup G. As a consequence, the original global $\mathrm{G} \times \mathrm{G}$-symmetry is broken to the diagonal G-symmetry as soon as the deformation is switched on. In the present case it is less obvious than for $\Phi_{\mathrm{G} \times \mathrm{G}}(z, \bar{z})$ but again we need to impose the vanishing of the Killing form since otherwise the field $\Phi_{\mathrm{G}}(z, \bar{z})$ would break conformal invariance at higher orders in perturbation theory. Similarly to the previous case, perturbations of a supergroup WZW model by the field (3.57) give rise to a non-chiral current algebra which was the subject of [112.

In the case of the $\operatorname{OSP}(m \mid 2 n)$ model at level $k=1$ that was discussed in Section 3.4, a slight modification of $\Phi_{\mathrm{G}}$ will play an important role below. In order to define it, we introduce the involutive automorphism $\Omega$ such that the fixed point set $\{X \in \operatorname{osp}(m \mid 2 n) \mid \Omega(X)=X\}$ is isomorphic to $\operatorname{osp}(m-1 \mid 2 n)$. On the basis we introduced in (3.40), $\Omega$ acts non-trivially only on $E_{i j}, T_{i a}, \bar{T}_{i a}$. In fact, it multiplies all operators with $i=1$ by -1 and leaves the others invariant. If we denote the anti-holomorphic fields corresponding to $\psi_{i}, \beta_{a}, \gamma_{a}$ by $\bar{\psi}_{i}, \bar{\beta}_{a}, \bar{\gamma}_{a}$, the deformation operator $\Phi_{\mathrm{G}}^{\prime}=J^{\mu} \Omega\left(\bar{J}_{\mu}\right)$ can then be written explicitly as

$$
J^{\mu} \Omega\left(\bar{J}_{\mu}\right)=\frac{1}{2}\left[\sum_{i=1}^{m} \varpi_{i} \psi_{i} \bar{\psi}_{i}+\sum_{a=1}^{n}\left(\gamma_{a} \bar{\beta}_{a}-\beta_{a} \bar{\gamma}_{a}\right)\right]^{2}
$$

where $\varpi=(-1,1, \ldots, 1)$. In order for the expression on the right hand side line to make sense, we need to first expand the square and normal order the products. Without the contributions from the bosonic ghosts, the interaction introduced by $\Phi_{\mathrm{G}}^{\prime}$ is familiar from Gross-Neveu models, i.e. the deformed OSP WZW model can be regarded as a Gross-Neveu model. As we have argued, this Gross-Neveu model is conformally invariant if $m=2 n+2$, i.e. if the Killing form of $\operatorname{osp}(m \mid 2 n)$ vanishes. Our construction thus generalizes the massless Thirring model (appearing, e.g., in Luttinger liquid theory) to a non-abelian setting.

\subsubsection{Exact marginality for quasi-abelian supergroups}

The previous two sections showed that a vanishing Killing form of $G$ is a necessary prerequisite for the existence of marginal deformation preserving either the symmetry $G$ or even the $G \times G$. We will refer to such supergroups $G$ as being quasi-abelian. This name is motivated by the equation

$$
0=K\left(T^{\mu}, T^{\nu}\right)=-(-1)^{d_{\lambda}} f_{\lambda}^{\mu \rho} f_{\rho}^{\nu \lambda}
$$

for the Killing form. We see that - even though the structure constants themselves are not vanishing - every contraction over two of its indices leads to a trivial result. This is also depicted in Figure 3 , One can easily convince oneself that the Killing form coincides with the Ricci curvature of G up to a factor. Hence quasi-abelian supergroups are Ricci flat (and vice versa).

Assuming the existence of a unique invariant rank three tensor (the structure constants), the $\beta$-function equations for G-preserving deformations of supergroup WZW models on G or for the 
corresponding principal chiral models follow the combinatorics for free bosons since all structure constants can be dropped [23]. This can be understood as follows. Since the G-symmetry is preserved, the $\beta$-function is a scalar quantity without free Lie algebra indices. On the other hand, the perturbation series for the $\beta$-function will be based on the OPE (3.2). The only ingredients are the metric (corresponding to a line) and the structure constants (corresponding to a 3-vertex). Let us pick out an arbitrary diagram which includes a structure constant $f_{\lambda}^{\mu \nu}$. It needs to be connected to the rest of the diagram by three legs. According to our assumption, the remaining diagram thus also needs to be proportional to the structure constants and hence the concatenation vanishes due to eq. (3.59). As a result we are left with the purely abelian contributions which are known to vanish. This reasoning is illustrated in Figure 3.

An alternative and less restrictive argument for the conformal invariance of deformed supergroup WZW models was provided in [113. It solely rests on the general non-chiral structure of the bulk state space and on the vanishing of the dual Coxeter number. The key idea is that the perturbing field $\Phi(z, \bar{z})=\Phi_{\mathrm{G} \times \mathrm{G}}(z, \bar{z})$ transforms as a singlet $\mathcal{L}_{0}$ under the right action of the supergroup G. From the general analysis in Section 3.3.3 we know that such a singlet can only arise as the socle of an atypical projective cover $\mathcal{P}_{0}$. For this reason there exists a SUSY partner $Q(z, \bar{z})$ and a fermionic symmetry operation $R$ such that $\Phi(z, \bar{z})=R Q(z, \bar{z})$. This statement may be used to show that all $N$-point functions of the perturbing field $\Phi(z, \bar{z})$ vanish identically. Indeed, in the correlation function

$$
\left\langle\Phi(z, \bar{z}) \Phi\left(w_{1}, \bar{w}_{1}\right) \cdots \Phi\left(w_{n}, \bar{w}_{n}\right)\right\rangle
$$

one can simply replace the first instance of $\Phi(z, \bar{z})$ by $R Q(z, \bar{z})$ and then use the Ward identities for the G-symmetry to redistribute $R$ to the other fields where it acts trivially. We conclude that there is no need to renormalize the field $\Phi(z, \bar{z})$ and this proves its exact marginality to all orders in perturbation theory. Another approach uses the representation of $\mathrm{G}$ as a symmetric space $\mathrm{G} \times \mathrm{G} / \mathrm{G}$ and cohomological reduction, see also Section 4.1.2

\subsubsection{Anomalous dimensions}

The perturbations discussed in the previous sections break the affine symmetry of the WZW model. For this reason, the rigid structure of affine modules is broken up, leading to a drastic deformation of the spectrum. The latter is most conveniently expressed in terms of anomalous dimensions, i.e. the difference of the conformal weight compared to the unperturbed theory (in this case the WZW model, not a free field theory) as a function of the coupling $\xi$. While generally valid formulas for the anomalous dimensions are currently still lacking, quasi-abelian perturbation theory has enabled progress in certain special situations which shall now briefly be reviewed.

First of all, perturbations of the form (3.56) which preserve the global $\mathrm{G} \times \mathrm{G}$-symmetry of the WZW model are very hard to deal with due to the involvement of the field $\phi_{\mu \nu}(z, \bar{z})$. A simplification only occurs if one is considering the boundary spectrum of a D0-brane. In that case, the field $\phi_{\mu \nu}(z, \bar{z})$ effectively becomes localized and may be replaced by a constant [113. Under these circumstances, the perturbation series for the boundary two-point functions may be summed up completely to all orders for a certain subset of fields, and it leads to a "Casimir evolution" where the anomalous dimensions are proportional to the Casimir $C_{\lambda}$ of the representation under consideration. The Casimir is multiplied by a geometric series in the coupling constant $\xi$ which resembles the result for the radius deformation of a free field theory. Surprisingly, the anomalous dimension only depends on the transformation properties of the field under the global symmetry $\mathrm{G}$ but not on its original location in the affine representation of the WZW model.

The situation is more favorable in case of G-preserving deformations. In that case, formulas for bulk and boundary anomalous dimensions have been proposed in [39, 114, 115], based on similar ideas. In both cases, one recovers a Casimir evolution which, for the boundary case, leads to the formula

$$
\delta h_{\lambda}(\xi)=-\frac{k \xi}{1+k \xi} \frac{C_{\lambda}}{k} .
$$

Here, $C_{\lambda}$ refers to the (generalized) eigenvalue of the quadratic Casimir acting on the representation $\lambda$. The recent analysis in [115] proves formula (3.61) for a specific subset of fields. Even 
though no general proof for generic fields exists, formula (3.61) has been backed up in several models by numerical checks in lattice discretizations [100, 82. Assuming its general validity leads to remarkable statements about spectral equivalences between deformed WZW models and supersphere $\sigma$-models which are likely too non-trivial to be just accidental. A more detailed discussion of these conjectural dualities can be found in Section 4.3. We finally wish to mention that the combination of Casimir evolution with cohomological reduction has been used in [82] in order to propose a formula for boundary spectra of space-filling branes with monopole charge in $\mathbb{C P}^{1 \mid 2}$.

\section{Superspace sigma models and strings}

Up to now our discussion of logarithmic conformal field theories with internal supersymmetry was focused on WZW models and their deformations. On the other hand, applications e.g. to string theory in Anti-de Sitter backgrounds, often involve superspace $\sigma$-models. These will be the focus of the final chapter. After reviewing the definition of $\sigma$-models we shall discuss existing results on conformal invariance of these model. We continue with some comments on embeddings into string theory using either the hybrid or pure spinor approach. In the third subsection we study the $\sigma$-model on the supersphere $\mathrm{S}^{3 \mid 2}$ and establish an intriguing link with an $\operatorname{OSP}(4 \mid 2)$ WZW model at level $k=1$.

\subsection{Sigma models on coset superspaces}

\subsubsection{Basic formulation}

We want to consider non-linear $\sigma$-models on homogeneous superspaces $\mathrm{G} / \mathrm{G}^{\prime}$, where the quotient is defined as the set of right cosets of $G^{\prime}$ in $G$ through the identification

$$
g \sim g h \text { for all } h \in \mathrm{G}^{\prime} \subset \mathrm{G}
$$

Let $\mathfrak{g}$ be the Lie superalgebra associated to G. We assume that $\mathfrak{g}$ comes equipped with a nondegenerate invariant bilinear form $\langle\cdot, \cdot\rangle$. Examples include $\mathfrak{g}=\operatorname{gl}(m \mid n), \operatorname{sl}(m \mid n), \operatorname{psl}(n \mid n)$ or $\operatorname{osp}(m \mid 2 n) 21$ Similarly, let $\mathfrak{g}^{\prime}$ be the Lie superalgebra associated to $\mathrm{G}^{\prime}$. We assume that the restriction of $\langle\cdot, \cdot\rangle$ to $\mathfrak{g}^{\prime}$ is non-degenerate. In this case, the orthogonal complement $\mathfrak{m}$ of $\mathfrak{g}^{\prime}$ in $\mathfrak{g}$ is a $\mathfrak{g}^{\prime}$-module and one can write the following $\mathfrak{g}^{\prime}$-module decomposition $\mathfrak{g}=\mathfrak{g}^{\prime} \oplus \mathfrak{m}$. In particular, this means that there are projectors $P^{\prime}$ onto $\mathfrak{g}^{\prime}$ and $P=1-P^{\prime}$ onto $\mathfrak{m}$ which commute with the action of $\mathfrak{g}^{\prime}$.

With the above requirements, the quotient $\mathrm{G} / \mathrm{G}^{\prime}$ can be endowed with a G-invariant metric $\mathrm{g}$. This metric is by no means unique and generally depends on a number of continuous parameters. The square root of the superdeterminant of $g$ provides in the standard way a G-invariant measure $\mu$ on $\mathrm{G} / \mathrm{G}^{\prime}$. With these two structures one can already write down a purely kinetic Lagrangian for the $\sigma$-model on $\mathrm{G} / \mathrm{G}^{\prime}$ and quantize it in the path integral formalism. Inclusion of $\theta$-terms, WZW terms or $B$-fields requires a better understanding of the geometry of the $\mathrm{G} / \mathrm{G}^{\prime}$ superspace. In fact, the $\theta$ and WZW terms are associated to G-invariant closed but not exact 2- and 3-forms, respectively. $B$-fields, on the other hand, are written in terms of G-invariant exact 2-forms. Every such linearly independent form comes with its own coupling constant. We shall only consider Lagrangians with a kinetic term and a $B$-field. Let b be some general G-invariant exact 2-form. Then the most general Lagrangian we consider can be written in the form

$$
\mathcal{L}=\eta^{a b} \mathrm{~g}_{\mu \nu}(\phi) \partial_{a} \phi^{\mu} \partial_{b} \phi^{\nu}+\epsilon^{a b} \mathrm{~b}_{\mu \nu}(\phi) \partial_{a} \phi^{\mu} \partial_{b} \phi^{\nu}
$$

where $\eta^{a b}$ is the constant world sheet metric, $\epsilon^{a b}$ the antisymmetric tensor with $\epsilon^{01}=1$. The Lagrangian is obviously evaluated on maps $\phi$ from the worldsheet $\Sigma$ to the superspace $\mathrm{G} / \mathrm{G}^{\prime}$.

There is a different way to formulate the $\sigma$-model on $\mathrm{G} / \mathrm{G}^{\prime}$, which makes its coset nature manifest and allows to explicitly construct the metric $g$ and the $B$-field $b$ in eq. (4.2). For that purpose, instead of maps $\phi$ from the worldsheet to the target space $G / G^{\prime}$, we consider more general

\footnotetext{
${ }^{21}$ We exclude $\operatorname{sl}(n \mid n)$ and $\operatorname{pgl}(n \mid n)$, since they do not have a non-degenerate metric.
} 
maps $g: \Sigma \rightarrow$ G from the world sheet to the Lie supergroup G. A basis set of 1-forms on $\mathrm{G}$ which are invariant under the global left G-action is provided by the so-called Maurer-Cartan forms

$$
J(x)=g^{-1}(x) d g(x)=\left[\omega_{\mu}^{i}(\phi) H_{i}+E_{\mu}^{m} X_{m}\right] d \phi^{\mu} .
$$

Here $H_{i}, i=1, \ldots, \operatorname{dim} \mathfrak{g}^{\prime}$, denotes a basis in the Lie superalgebra $\mathfrak{g}^{\prime}$ and $X_{m}$ is one for the complement of $\mathfrak{g}^{\prime}$ in $\mathfrak{g}$. The coefficient functions $\omega_{\mu}^{i}$ and $E_{\mu}^{m}$ are the spin connection and the vielbein, respectively. In terms of these currents, we can write

$$
\mathcal{L}=\eta^{a b} \mathrm{G}\left\langle P\left(J_{a}\right), P\left(J_{b}\right)\right\rangle+\epsilon^{a b} \mathrm{~B}\left\langle P\left(J_{a}\right), P\left(J_{b}\right)\right\rangle,
$$

where $P: \mathfrak{g} \rightarrow \mathfrak{m}=\mathfrak{g} / \mathfrak{g}^{\prime}$ is the projection and

$$
\mathrm{G} \in \operatorname{Hom}_{\mathfrak{g}^{\prime}}(\mathfrak{m} \circ \mathfrak{m}, \mathbb{C}) \quad \text { and } \quad B \in \operatorname{Hom}_{\mathfrak{g}^{\prime}}(\mathfrak{m} \wedge \mathfrak{m}, \mathbb{C})
$$

are taken from the symmetric, respectively antisymmetric tensor product of $\mathfrak{m}$ with itself to the trivial representation. The relation with eq. (4.2) is provided by

$$
\mathrm{g}_{\mu \nu}(\phi)=\mathrm{G}_{m n} E_{\mu}^{m}(\phi) E_{\nu}^{n}(\phi) \quad, \quad \mathrm{b}_{\mu \nu}(\phi)=\mathrm{B}_{m n} E_{\mu}^{m}(\phi) E_{\nu}^{n}(\phi) .
$$

Any model of the form (4.4) may be considered as a consistent $\sigma$-model on the coset space $\mathrm{G} / \mathrm{G}^{\prime}$. Under right $\mathrm{G}^{\prime}$-gauge transformations $g^{\prime}: \Sigma \mapsto \mathrm{G}^{\prime}$ the Maurer-Cartan forms $J_{\mu}$ transform as

$$
g(x) \mapsto g(x) g^{\prime}(x), \quad J_{\mu}(x) \mapsto g^{\prime}(x)^{-1} J_{\mu}(x) g^{\prime}(x)+g^{\prime}(x)^{-1} \partial_{\mu} g^{\prime}(x) .
$$

Since the projection $P$ on $\mathfrak{m}$ commutes with the action of $\mathfrak{g}^{\prime}$, the projected forms $P\left(J_{\mu}\right)$ transform by conjugation with $g^{\prime}$. Hence, the Lagrangian (4.4) is independent of how we choose representatives in the coset space $\mathrm{G} / \mathrm{G}^{\prime}$. Global left G-invariance of the Lagrangian (4.4) is automatic since Maurer-Cartan forms $J_{\mu}(x)$ are left G-invariant by construction.

\subsubsection{Symmetric superspaces and conformal invariance}

As we discussed in the previous paragraph, $\sigma$-models on coset spaces may be defined in terms of the basic objects (4.5). There are many examples. If we choose $\mathrm{G}=\mathrm{SO}(4)$ and $\mathrm{G}^{\prime}=\mathrm{SO}(3)$, the space $\mathfrak{m}$ is 3 -dimensional and it transforms in the adjoint representation of $\mathrm{SO}(3)$. In this case, there exists a single invariant in the symmetric tensor product of $\mathfrak{m}$ with itself and no invariant in the anti-symmetric case. Hence, there is a one-parameter family of metrics $G$ and no B field. The corresponding family of $\sigma$-models has a target space $\mathrm{S}^{3}=\mathrm{SO}(4) / \mathrm{SO}(3)$ with radius $R$. Because of the non-vanishing curvature, the model is not conformal, unless we add a WZW term.

A very similar analysis applies to the pair $\mathrm{G}=\mathrm{OSP}(4 \mid 2)$ and $\mathrm{G}^{\prime}=\operatorname{OSP}(3 \mid 2)$, only that in this case the $\sigma$-model on the quotient $\mathrm{S}^{3 \mid 2}=\operatorname{OSP}(4 \mid 2) / \operatorname{OSP}(3 \mid 2)$ turns out to be conformal. In the case of symmetric superspaces a complete list of conformal invariant $\sigma$-models is actually known. For simplicity, let us assume that $\mathrm{G}$ is simple. Then the $\sigma$-model on the symmetric superspace $\mathrm{G} / \mathrm{G}^{\prime}$, where $\mathrm{G}^{\prime}=\mathrm{G}^{\mathbb{Z}_{2}}$ is a subgroup $\mathrm{G}^{\prime} \subset \mathrm{G}$ of elements that are left invariant by the action of an automorphism of order two on $\mathrm{G}$, and $\mathrm{B}=0$ is conformal if and only if

$$
\begin{aligned}
& C_{\mathrm{G}}^{(2)}(\mathfrak{g})=0 \\
& C_{\mathrm{G}_{\sigma}^{\prime}}^{(2)}(\mathfrak{m})=0
\end{aligned}
$$

Here $C_{\mathrm{G}}^{(2)}(L)$ denotes the value of the quadratic Casimir element of $\mathrm{G}$ on the module $L$ and we have decomposed $\mathrm{G}^{\prime}$ into simple factors $\mathrm{G}^{\prime}=\prod_{\sigma} \mathrm{G}_{\sigma}^{\prime}$. The first condition on the dual Coxeter number $g^{\vee}=C_{\mathrm{G}}^{(2)}(\mathfrak{g}) / 2$ of the numerator supergroup $\mathrm{G}$ guarantees that the perturbative beta function vanishes at one loop. In fact, the Ricci tensor of a coset space $G / G^{\prime}$ is given by

$$
R_{m n}\left(\mathrm{G} / \mathrm{G}^{\prime}\right)=2 R_{m n}(\mathrm{G})+\frac{1}{4}(-1)^{|k|} f_{m l}^{k} f_{n k}^{l} .
$$

We shall use in addition that

$$
R_{m n}(\mathrm{G})=-\frac{1}{4} K_{m n}^{\mathrm{G}}=-\frac{1}{4}(-1)^{|D|} f_{m C}^{D} f_{n D}^{C} .
$$


Here, capital letters $C, D$ run over a basis in $\mathfrak{g}$ while lower case letters $m, n$ are restricted to basis elements in $\mathfrak{m} \subset \mathfrak{g}$. For symmetric superspaces, the structure constants of $\mathrm{G}$ must be consistent with the $\mathbb{Z}_{2}$ automorphism and hence $f^{k}{ }_{m l}=0$. Consequently, the Ricci tensor for symmetric spaces is determined by the Killing form of $\mathrm{G}$. If the latter vanishes, so does the beta function at one loop. The condition (4.9) arises from the two loop beta function. It is possible to show that all models that pass this condition possess a vanishing beta functions to all loop order, see 101.

Let us note that the principal chiral model on a group or supergroup U could also be formulated as a symmetric space $\sigma$-model. Without any further thought one might be tempted to describe this model through $\mathrm{G}=\mathrm{U}$ and $\mathrm{G}^{\prime}=\{e\}$. But as our introductory comments suggest, we prefer to rewrite the group manifold $\mathrm{U}$ as a coset superspace $\mathrm{U}=\mathrm{U} \times \mathrm{U} / \mathrm{U}$ and hence to set

$$
\mathrm{G}=\{(x, y) \mid x, y \in \mathrm{U}\} \quad, \quad \mathrm{G}^{\prime}=\{(x, x) \mid x \in \mathrm{U}\} .
$$

The left and right action of $\mathrm{G}$ on itself is given by componentwise multiplication. The right coset superspace $\mathrm{G} / \mathrm{G}^{\prime} \cong \mathrm{U}$ is considered as the space of equivalence classes under the equivalence relation $(x, y) \sim(x z, y z)$, for all $z \in \mathrm{U}$. In particular, $\left(x y^{-1}, 1\right)$ is the canonical representative of the equivalence class of $(x, y)$. Hence, the currents $J_{\mu}$ and the projection map $P: \mathfrak{g} \rightarrow \mathfrak{m}$ are given by

$$
J_{\mu}=\left(x^{-1} \partial_{\mu} x, y^{-1} \partial_{\mu} y\right), \quad P:(v, w) \mapsto\left(\frac{v-w}{2},-\frac{v-w}{2}\right) .
$$

If $\langle\cdot, \cdot\rangle$ is the invariant form on the Lie superalgebra of $\mathrm{U}$ and we take $\mathrm{G}$ to be given by

$$
\mathrm{G}\left(\left(v_{1}, w_{1}\right) \otimes_{s}\left(v_{2}, w_{2}\right)\right)=\left\langle v_{1}, v_{2}\right\rangle+\left\langle w_{1}, w_{2}\right\rangle
$$

we obtain the usual principal chiral model for $\mathrm{U}$. In fact, one may easily show that

$$
\mathrm{G}\left(P\left(J_{\mu}\right), P\left(J_{\nu}\right)\right) \eta^{\mu \nu}=\frac{1}{2}\left(u^{-1} \partial_{\mu} u, u^{-1} \partial_{\nu} u\right) \eta^{\mu \nu}
$$

where $u=x y^{-1} \in \mathrm{U}$. Thereby we have established the standard geometric result that allows us to treat the principal chiral model on $\mathrm{U}$ as a $\mathrm{G} / \mathrm{G}^{\prime}$ coset superspace model.

\subsubsection{Remarks on $G / G^{\mathbb{Z}_{N}}$ coset superspaces}

While the results for conformal $\sigma$-models on symmetric superspaces are complete, much less is known for some of the extensions that appear e.g. in the context of AdS compactifications, see below. In fact, for many cases of interest, the Lie sub-superalgebra $\mathfrak{g}^{\prime}$ in $\mathfrak{g}$ consists of elements that are invariant under some automorphism $\Omega: \mathfrak{g} \rightarrow \mathfrak{g}$ of order $N>2$. An automorphism of order $N$ defines a decomposition

$$
\mathfrak{g}=\mathfrak{g}^{\prime} \oplus \bigoplus_{i=1}^{N-1} \mathfrak{m}_{i},\left.\quad \Omega\right|_{\mathfrak{g}^{\prime}}=1, \quad \Omega\left(\mathfrak{m}_{k}\right)=e^{\frac{2 \pi i}{N} k} \mathfrak{m}_{k}
$$

of the superalgebra $\mathfrak{g}$ into eigenspaces of $\Omega$. Extending our previous notation, we denote by $P_{i}$ the projection maps onto $\mathfrak{m}_{i}$. Thanks to the properties of $\Omega$, we find

$$
\left[\mathfrak{m}_{i}, \mathfrak{m}_{j}\right] \subset \mathfrak{m}_{i+j \bmod N},\left\langle\mathfrak{m}_{i}, \mathfrak{m}_{j}\right\rangle=0 \text { if } i+j \neq 0 \bmod N,
$$

where we have set $\mathfrak{m}_{0} \equiv \mathfrak{g}^{\prime}$. Consequently, the subalgebra $\mathfrak{g}^{\prime}$ acts on the $\Omega$-eigenspaces $\mathfrak{m}_{i}$. Note that the spaces $\mathfrak{m}_{i}$ need not be indecomposable under $\mathfrak{g}^{\prime}$ in which case the decomposition into $\mathfrak{g}^{\prime}$-modules is finer than the decomposition (4.15) into eigenspaces of $\Omega$.

Whenever a coset superspace $\mathrm{G} / \mathrm{G}^{\prime}$ is defined by an automorphism $\Omega$ of order $N$ we shall use the alternative notation $\mathrm{G} / \mathrm{G}^{\mathbb{Z}_{N}}$. The cases when the grading induced by $\Omega$ is compatible with the $\mathbb{Z}_{2}$ superalgebra grading, that is $\mathfrak{m}_{2 i} \in \mathfrak{g}_{\overline{0}}$ and $\mathfrak{m}_{2 i-1} \in \mathfrak{g}_{\overline{1}}$, were considered by Kagan and Young in [20]. They restricted to a family of Lagrangians for which $G$ and B take the following special form

$$
\mathrm{G}(X, Y)=\sum_{i=1}^{N-1} p_{i}\left\langle P_{i}(X), P_{N-i}(Y)\right\rangle \quad, \quad \mathrm{B}(X, Y)=\sum_{i=1}^{N-1} q_{i}\left\langle P_{i}(X), P_{N-i}(Y)\right\rangle
$$


where the $p_{i}$ and $q_{i}$ are constants obeying the additional constraints

$$
p_{i}=p_{N-i} \quad \text { and } \quad q_{i}=-q_{N-i} .
$$

The forms of $G$ and $B$ in eq. (4.17) do not give rise to the most general Lagrangian for coset superspaces $\mathrm{G} / \mathrm{G}^{\prime}$. As an example consider the famous $\mathbb{Z}_{4}$ quotient $\mathrm{PSU}(2,2 \mid 4) / \mathrm{SO}(1,4) \times \mathrm{SO}(5)$. Its metric has two radii because its bosonic base is $\operatorname{AdS}_{5} \times \mathrm{S}^{5}$. On the other hand, the special form of $\mathrm{G}$ in eq. (4.17) allows for only two parameters $p_{1}=p_{3}$ and $p_{2}$, among which $p_{1}$ is redundant because of the purely fermionic nature of $\mathfrak{m}_{1}$ and $\mathfrak{m}_{3}$. In this example, the form that $G$ takes in eq. (4.17) restricts the radii of $\mathrm{AdS}_{5}$ and $\mathrm{S}^{5}$ to be equal.

The properties of the theory defined by eqs. (4.17) certainly depend on the precise choice of the parameters $p_{i}$ and $q_{i}$. In particular, it was shown in [26] and [20] that one loop conformal invariance requires

$$
p_{i}=1 \quad q_{i}=1-\frac{2 i}{N} \quad \text { for } i \neq 0,
$$

for all even $N$. We believe, however, that in most cases these conditions are not sufficient to guarantee the vanishing of the full beta function.

\subsection{Embedding into string theory}

All superspace $\sigma$-models that appear in string theory are constructed in terms of generalized symmetric spaces for automorphisms of order $N=4$. Here we shall describe one of these models in the so-called hybrid approach before we give an overview over related models and their application to strings in AdS backgrounds.

\subsubsection{The coset space $\operatorname{PSU}(1,1 \mid 2) / \mathbf{U}(1) \times \mathbf{U}(1)$}

The coset space we want to look at in this subsection takes the form

$$
\mathrm{G} / \mathrm{G}^{\prime}=\operatorname{PSU}(1,1 \mid 2) / \mathrm{U}(1) \times \mathrm{U}(1) .
$$

We will show below that the denominator subgroup $G^{\prime}=U(1) \times U(1)$ is kept fixed by an order $N=4$ automorphisms of the numerator supergroup $\mathrm{G}=\mathrm{PSU}(1,1 \mid 2)$. The bosonic submanifold of the quotient is a product of two factors

$$
\operatorname{PSU}(1,1 \mid 2)^{(0)} / \mathrm{U}(1) \times \mathrm{U}(1)=\mathrm{SU}(1,1) / \mathrm{U}(1) \times \mathrm{SU}(2) / \mathrm{U}(1) .
$$

These two factors possess a simple geometric interpretation

$$
\begin{aligned}
\mathrm{SU}(2) / \mathrm{U}(1) & =\mathrm{SO}(3) / \mathrm{SO}(2)=\mathrm{S}^{2}=\left\{x_{1}^{2}+x_{2}^{2}+x_{3}^{2}=1\right\} \\
\mathrm{SU}(1,1) / \mathrm{U}(1) & =\mathrm{SO}(1,2) / \mathrm{SO}(1,1)=\mathrm{AdS}_{2}=\left\{y_{-1}^{2}+y_{0}^{2}-y_{1}^{2}=1\right\} .
\end{aligned}
$$

The Lie superalgebra $\operatorname{psl}(2 \mid 2)$ was first introduced in Section 2.1. It is generated by six bosonic elements $K_{a b}=-K_{b a}, a, b=0,1,2,3$ along with eight fermionic ones. These are denoted by $S_{a \alpha}, \alpha=1,2$ and they obey the relations

$$
\begin{aligned}
{\left[K_{a b}, K_{c d}\right] } & =\eta_{a c} K_{b d}-\eta_{a d} K_{b c}-\eta_{b c} K_{a d}+\eta_{b d} K_{a c} \\
{\left[K_{a b}, S_{c \alpha}\right] } & =\eta_{a c} S_{b \alpha}-\eta_{b c} S_{a \alpha} \\
{\left[S_{a \alpha}, S_{b \beta}\right] } & =\frac{1}{2} \epsilon_{a b c d} \epsilon_{\alpha \beta} K_{c d} .
\end{aligned}
$$

As we know from our discussion in Section 2.2 this Lie superalgebra possesses vanishing dual Coxeter number. Let us now define the action of $\mathbb{Z}_{4}$ on $\mathfrak{g}$ by

$$
\gamma\left(\mathfrak{m}_{r}\right)=e^{\frac{\pi i r}{2}} \mathfrak{m}_{r}
$$


where

$$
\begin{aligned}
& \mathfrak{m}_{0}=\left\langle K_{01}, K_{23}\right\rangle \subset \mathfrak{g}_{0} \\
& \mathfrak{m}_{1}=\left\langle S_{0 \alpha}+i S_{1 \alpha}, S_{2 \alpha}+i S_{3 \alpha}\right\rangle \subset \mathfrak{g}_{1} \\
& \mathfrak{m}_{2}=\left\langle K_{03}, K_{12}, K_{13}, K_{02}\right\rangle \subset \mathfrak{g}_{0} \\
& \mathfrak{m}_{3}=\left\langle S_{0 \alpha}-i S_{1 \alpha}, S_{2 \alpha}-i S_{3 \alpha}\right\rangle \subset \mathfrak{g}_{1} .
\end{aligned}
$$

It is easy to check that this definition respects the graded commutation relations (4.24) that define $\operatorname{psl}(2 \mid 2)$. The invariant subalgebra is generated by the two commuting $\mathrm{U}(1)$ charges $K_{01}$ and $K_{23}$. Hence, the coset (4.20) is a generalized symmetric superspace with an automorphism group of order $N=4$. Therefore, we can apply the results discussed in the previous subsection to build a conformally invariant $\sigma$-model, at least to one loop order.

\subsubsection{String theory on $\mathrm{AdS}_{2} \times \mathrm{S}^{2} \times \mathrm{CY}_{6}$}

In this subsection we want to illustrate in one example how to embed one of our $\sigma$-models into a full string background. For the $\mathrm{AdS}_{2} \times \mathrm{S}^{2} \sigma$-model this was explained by Berkovits et al. in [19]. The proposal is to add any $\sigma$-model on a Calabi-Yau superspace $\mathrm{CY}_{6}$ along with an additional free boson $\rho$. Let us discuss these ingredients separately. The factors $\mathrm{AdS}_{2} \times \mathrm{S}^{2}$ bring in a $\sigma$-model on $\operatorname{PSU}(1,1 \mid 2) / \mathrm{U}(1) \times \mathrm{U}(1)$. According to our general discussion in the previous subsection, this model has a single free parameter once we require conformal invariance,

$$
S_{\mathrm{G} / \mathrm{G}^{\prime}}=\frac{R^{2}}{4 \pi} \int d^{2} z\left(\left\langle J^{(2)}, \bar{J}^{(2)}\right\rangle+\frac{3}{2}\left\langle J^{(1)}, \bar{J}^{(3)}\right\rangle+\frac{1}{2}\left\langle J^{(3)}, \bar{J}^{(1)}\right\rangle\right) .
$$

As we discussed above, the beta function of this theory vanishes at one loop. We believe that in this particular case, it vanishes to all loops. It would be interesting to check this claim directly.

Next, let us turn to the free boson $\rho$. This boson is assumed to be compactified to the self-dual radius and to possess time-like signature

$$
\rho(z, \bar{z}) \rho(w, \bar{w}) \sim-\log |z-w|^{2} .
$$

As usual, we can decompose $\rho(z, \bar{z})$ into its chiral components $\rho(z, \bar{z})=\rho(z)+\bar{\rho}(z)$ and build vertex operators of the form

$$
V(z)=e^{i \rho(z)} \quad, \quad \bar{V}(\bar{z})=e^{i \bar{\rho}(\bar{z})} .
$$

These are fermionic local (anti-)holomorphic operators of conformal weight $(h, \bar{h})=(-1 / 2,0)$ and $(h, \bar{h})=(0,-1 / 2)$.

Finally, $\mathrm{CY}_{6}$ can stand for any conformal field theory with an $N=2$ superconformal symmetry of central charge $c=9$. Sigma models on Calabi-Yau manifolds of complex dimension $d=3$ provide many examples. Exact conformal field theories with the desired properties have been obtained through Gepner's famous construction [116.

Assuming that the $\sigma$-model on the coset superspace (4.20) is conformal the total central charge of the resulting theory is

$$
c^{\text {tot }}=(-2-1-1)+1+9=6
$$

Berkovits et al. were able to construct an $N=2$ superconformal algebra out of these models. For the $\sigma$-model on $\mathrm{CY}_{6}$ this comes with the Calabi-Yau condition. The challenge was therefore to build one out of the $\sigma$-model on the superspace (4.20) and the boson $\rho$. The bosonic elements take the form

$$
T=T_{\mathrm{G} / \mathrm{G}^{\prime}}+\frac{1}{2} \partial \rho \partial \rho+T_{\mathrm{CY}} \quad, \quad J=i \partial \rho+J_{\mathrm{CY}}
$$

and there exist two fermionic currents $G^{ \pm}$in which the boson $\rho$ is coupled to a particular holomorphic combination of the currents $S_{a \alpha}$, see [19] for details. 
The string spectrum is obtained by gauging the $N=2$ superconformal algebra. To this end one introduces fermionic/bosonic ghosts for the bosonic/fermionic fields $T, J / G^{ \pm}$that form the $N=2$ algebra. The $b / \beta$ ghosts of these four ghost systems must possess conformal weights $h_{b^{T}}=2, h_{b^{J}}=1$ and $h_{\beta^{ \pm}}=3 / 2$. Given the usual rules for computing the central charge,

$$
c_{b}=-3\left(2 h_{b}-1\right)^{2}+1 \quad \text { and } \quad c_{\beta}=3\left(2 h_{\beta}-1\right)^{2}-1,
$$

for $b=b^{T}, b^{J}$ and $\beta=\beta^{ \pm}$, the total $c^{\text {gh }}$ of the four ghost systems adds up to

$$
c^{\mathrm{gh}}=-26-2+2 \times 11=-6 .
$$

Hence, the sum of the central charges (4.33) from the matter sector and $c^{\text {gh }}$ from the ghost sector add up to $c=0$. The BRST operator for the gauging of an $N=2$ superconformal algebra is given in [117. When the supercoset $\mathrm{G} / \mathrm{G}^{\prime}$ is replaced by a 4-dimensional Minkowski space, the cohomology of the corresponding BRST operator may be shown to coincide with the spectrum of a Calabi-Yau compactification of type II superstring theory 118 . In the case of the $\sigma$-model on $\operatorname{PSU}(1,1 \mid 2) / \mathrm{U}(1)^{2}$, the cohomology of $Q_{\mathrm{BRST}}$ provides the spectrum of type II superstring theory on $\mathrm{AdS}_{2} \times \mathrm{S}^{2} \times \mathrm{CY}_{6}$. Note that the underlying model is just a product of the superspace $\sigma$-model with the chiral boson and the $c=9$ CFT describing strings on $\mathrm{CY}_{6}$. These models are only coupled through the BRST operator.

\subsubsection{Other string backgrounds - an overview}

A discussion similar to the one we have outlined here for strings in $\mathrm{AdS}_{2} \times \mathrm{S}^{2}$ can be performed for $\mathrm{AdS}_{3} \times \mathrm{S}^{3}$. The associated $\mathbb{Z}_{4}$ coset model is given by $\mathrm{G} / \mathrm{G}^{\prime}=\mathrm{PSU}(1,1 \mid 2)^{2} / \mathrm{SO}(1,2) \times \mathrm{SO}(3)$, see [19. The full model requires two chiral bosons and a number of ghost fields for harmonic constraints. The latter may be used to reduce the model to an alternative formulation involving a $\sigma$-model on $\operatorname{PSU}(1,1 \mid 2)$ that was described first in $[119$. Conformal invariance of the $\sigma$-model on $\operatorname{PSU}(1,1 \mid 2)$ has been established to all orders in $\left[22\right.$, 23. In case of $\operatorname{AdS}_{3} \times \mathrm{S}^{3}$ it is possible to switch on an NSNS 3-form flux $H$ in addition to the RR-flux that is usually considered to obtain a consistent string background. On the world-sheet, the NSNS-flux corresponds to a (bosonic) WZW term in the $\sigma$-model on PSU $(1,1 \mid 2)$. This model is known to be classically integrable [120] and quantum conformally invariant. At the so-called WZ point, the theory possesses additional holomorphic currents that render it solvable by standard conformal field theory techniques. The solution has been worked out in [24, using methods similar to the ones presented in Section 3.2 . With the WZW model being under good control, it has been possible to analyse the spectrum of physical states, i.e. the cohomology of the BRST operator of the hybrid formalism, see [121] for the massless spectrum and 122 for an extension to massive states. As has been argued in a toy model by Troost 123 the resulting spectrum is a direct sum of irreducibles.

For higher dimensional AdS backgrounds with maximal supersymmetry, i.e. $\mathrm{AdS}_{5} \times \mathrm{S}^{5}$ and $\mathrm{AdS}_{4} \times \mathbb{C P}^{3}$, covariant string theoretic models may be constructed in the so-called pure spinor formalism. In the case of $\mathrm{AdS}_{5}$, the model was first proposed by Berkovits 124. Once again, it is based on a $\sigma$-model with a generalized symmetric target space $\mathrm{G} / \mathrm{G}^{\prime}=\mathrm{PSU}(2,2 \mid 4) / \mathrm{SO}(1,4) \times$ $\mathrm{SO}(5)$ where the denominator is kept fixed by an automorphism of order $N=4$. In this case, one needs to add 16 bosonic ghost fields subject to five independent pure spinor constraints. The pure spinor sector alone would hence contribute a total central charge $c^{\text {gh }}=32-10=22$. In the action of the pure spinor string theory, the ghost sector and the $\sigma$-model are coupled and once the interaction terms are included, the combined theory is believed to be conformal to all loop orders [125]. Its central charge $c=-22+22=0$ is obtained by adding the superdimension $\operatorname{sim} \mathrm{G} / \mathrm{G}^{\prime}=-2-10-10=-22$ to the central charge of the ghost sector. Classical integrability of the model was established in [126]. Properties of the monodromy of the Lax connection were studied more recently, see 127 and references therein. Similar developments exist in the case of $\mathrm{AdS}_{4} \times \mathbb{C P}^{3}$. In the latter case, the pure spinor model is based on the generalized symmetric superspace $\mathrm{G} / \mathrm{G}^{\prime}=\mathrm{OSP}(6 \mid 2,2) / \mathrm{U}(3) \times \mathrm{SO}(1,3)[128]$.

Before we conclude this short overview on superspace $\sigma$-models in exact string backgrounds, we would like to add a few comments on the relation with the Green-Schwarz formalism. For all the backgrounds we discussed in the previous two paragraphs, a corresponding Green-Schwarz 
description is known. In the case of $\mathrm{AdS}_{5} \times \mathrm{S}^{5}$ this was proposed by Metsaev and Tseytlin, see [18]. Similar models exist for $\mathrm{AdS}_{2} \times \mathrm{S}^{2} \times T^{6}$, see [129] and references therein, $\mathrm{AdS}_{3} \times \mathrm{S}^{3}[130$ ] and $\mathrm{AdS}_{4} \times \mathbb{C P}^{6}[131$. All these models employ $\sigma$-models on the very same generalized symmetric superspaces we have described before. On the other hand, the fermionic WZ terms need to be readjusted compared to the values that appeared in our discussion of one-loop conformal invariance. For consistency of the Green-Schwarz superstring, $\kappa$ symmetry is crucial. This feature requires a degenerate fermionic metric with the parameter $p_{1}=0$ rather than $p_{1}=1$ as in the case of the pure spinor formalism. After $\kappa$ symmetry has been fixed, one obtains conformal field theories with $c=26$ and for physical state selection one needs to impose the usual Virasoro constraints. All consistent AdS backgrounds that can be obtained in this way have been classified in [132. In all known examples the Green-Schwarz and pure spinor/hybrid formulations are believed to be equivalent, see 133 for a recent analysis at one loop.

\subsection{A duality between Gross-Neveu and supersphere sigma models}

Most of the results we have reported about in this review deal with WZW models and their deformations. On the other hand, $\sigma$-models certainly play an important role for applications in physics. Hence it is of interest to understand the possible relations between these two types of models. In string theory, there exists a beautiful relation between $\sigma$-models on Calabi-Yau spaces and so-called Gepner models [116. The latter are obtained from products of WZW coset models and both types of models are related by exactly marginal deformations 134. Therefore, one may think of the non-geometric Gepner models as describing string-size Calabi-Yau compactifications. Witten's explanation of the duality between Gepner-models and Calabi-Yau $\sigma$-models heavily relies on unitarity and hence no such techniques are at our disposal in the context of conformal field theories with internal supersymmetry.

On the other hand, a very similar duality between OSP $(2 S+2 \mid 2 S)$ Gross-Neveu models and $\sigma$ models on the superspheres $S^{2 S+1 \mid 2 S}$ has been proposed in [100] and then supported by a smooth interpolation of certain boundary partition functions [39. In the following we shall recall the formulation of the Gross-Neveu model as a deformed WZW model and explain how boundary spectra can be calculated along the lines of Section 3.5. For simplicity we restrict ourselves to the case of $S=1$. Then we deform the boundary spectrum away from the WZW point until we reach the large volume limit in which the partition function describes the counting of operators for a $\sigma$-model on $\mathrm{S}^{3 \mid 2}$. We think of the relation between the Gross-Neveu and the $\sigma$-model as a prototype of a large class of similar dualities for non-unitary models with internal supersymmetry. It is quite feasible that similar relations also exist for $\sigma$-models on the coset spaces we listed in the previous subsection. If this were the case, it could play a central role in a possible world-sheet derivation of Maldacena's AdS/CFT correspondence.

\subsubsection{The $\operatorname{OSP}(2 S+2 \mid 2 S)$ Gross-Neveu model}

We are now presenting arguments in favor of a duality between non-linear $\sigma$-models on superspheres $S^{2 S+1 \mid 2 S}$ and $\operatorname{OSP}(2 S+2 \mid 2 S)$ Gross-Neveu models 39. In the case $S=0$, this duality reduces to the well-known correspondence between the massless Thirring model (also known as Luttinger liquid in the condensed matter community) and the free compactified boson. All cases $S \geq 1$ can be thought of as non-abelian generalizations of this equivalence.

The OSP (4|2) Gross-Neveu model is a non-geometric theory defined by the following Lagrangian

$$
\mathcal{S}^{\mathrm{GN}}[\Psi]=\frac{1}{2 \pi} \int d^{2} z\left[\langle\Psi, \bar{\partial} \Psi\rangle+\langle\bar{\Psi}, \partial \bar{\Psi}\rangle+g^{2}\langle\Psi, \bar{\Psi}\rangle^{2}\right] .
$$

Here, $\Psi=\left(\psi_{1}, \ldots, \psi_{4}, \beta, \gamma\right)$ is a fundamental $\operatorname{OSP}(4 \mid 2)$ multiplet with four fermions and two bosons, all having conformal dimension $h=1 / 2$, as discussed in Section 3.4. The theory has a single coupling constant $g$ which determines the strength of the quartic potential. According to our discussion in Section 3.4 we can think of the OSP(4|2) Gross-Neveu model as a deformed OSP(4|2) WZW model at level $k=1$ and construct the interaction in terms of the currents as

$$
\mathcal{S}^{\mathrm{GN}}=\mathcal{S}^{\mathrm{WZW}}+g^{2} \mathcal{S}_{\mathrm{def}} \quad \text { with } \quad \mathcal{S}_{\mathrm{def}}=\frac{1}{2 \pi} \int d^{2} z\langle J, \Omega(\bar{J})\rangle,
$$




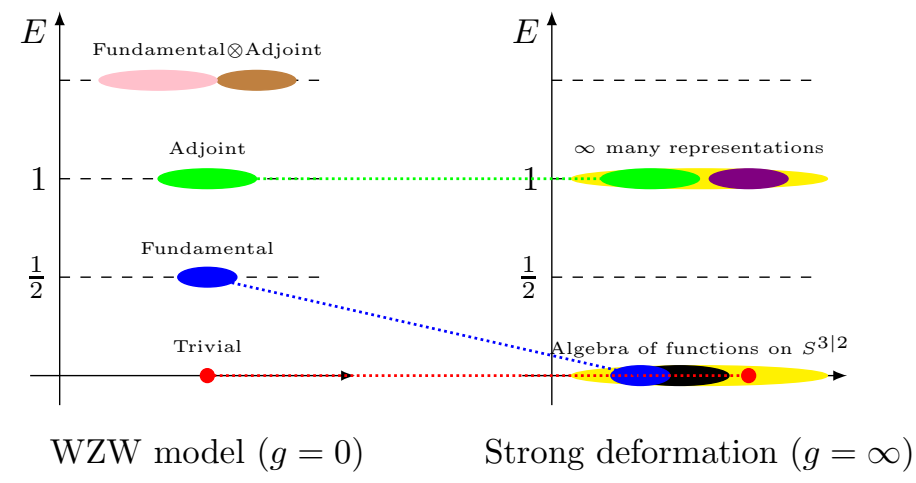

Figure 4: (Color online) A distinguished boundary spectrum of the OSP(4|2) Gross-Neveu model at zero and infinite coupling. The interpolation between these two spectra for other values of the coupling $g$ is described by formula (4.43).

where $\Omega$ is induced from the exchange automorphism of $\widehat{\mathrm{SU}}(2)_{1} \times \widehat{\mathrm{SU}}(2)_{1}$. This kind of deformation is covered by our discussion in Section 3.5.2.

In Section 3.4 we discussed the spectrum of the OSP(4|2) WZW model in the bulk. Alternatively, this theory can be formulated as an orbifold WZW model

$$
\widehat{\mathrm{OSP}}(4 \mid 2)_{1}=\left(\widehat{\mathrm{SU}}(2)_{-\frac{1}{2}} \times \widehat{\mathrm{SU}}(2)_{1} \times \widehat{\mathrm{SU}}(2)_{1}\right) / \mathbb{Z}_{2}
$$

of purely bosonic WZW models. The two copies of $\mathrm{SU}(2)_{1}$ arise from the two pairs of fermions, while the $\widehat{\mathrm{SU}}(2)_{-\frac{1}{2}}$ arises from the bosonic $\beta \gamma$ system. Now we want to place the theory on the upper half-plane and impose $\operatorname{OSP}(4 \mid 2)$ symmetry preserving boundary conditions. These are associated to trivial gluing conditions in the $\widehat{\mathrm{SU}}(2)_{-\frac{1}{2}}$ part and permutation gluing conditions in the $\widehat{\mathrm{SU}}(2)_{1} \times \widehat{\mathrm{SU}}(2)_{1}$ factors. It turns out that there is a unique boundary condition whose spectrum takes the form [39]

$$
Z_{\mathrm{GN}}(q, y \mid 0)=\frac{\eta(q)}{\theta_{4}\left(y_{1}\right)}\left[\frac{\theta_{2}\left(q^{2}, y_{2}^{2}\right) \theta_{2}\left(q^{2}, y_{3}^{2}\right)}{\eta(q)^{2}}+\frac{\theta_{3}\left(q^{2}, y_{2}^{2}\right) \theta_{3}\left(q^{2}, y_{3}^{2}\right)}{\eta(q)^{2}}\right] .
$$

It is just the sum of the affine $\widehat{\mathrm{OSP}}(4 \mid 2)_{1}$ characters based on the trivial and the fundamental representation of $\operatorname{OSP}(4 \mid 2)$.

\subsubsection{Deformed boundary spectrum}

Once the WZW model has been solved, it is straightforward to determine the deformed boundary spectrum using the results of Section 3.5.2 According to formula (3.61) the anomalous dimensions of a boundary field only depend on the transformation properties with respect to the global OSP(4|2)-symmetry. In order to use this formula, we must first decompose the spectrum (4.40) at the WZW point into osp(4|2) multiplets,

$$
Z_{\mathrm{GN}}\left(q, y \mid g^{2}=0\right)=\sum_{\Lambda} \psi_{\Lambda}^{\mathrm{WZW}}(q) \chi_{\Lambda}(y)
$$

Here, $\Lambda$ runs over weights of finite dimensional representations of $\operatorname{osp}(4 \mid 2), \chi_{\Lambda}(y)=\chi_{\Lambda}\left(y_{1}, y_{2}, y_{3}\right)$ denote the associated characters and $\psi_{\Lambda}^{\text {WZW }}$ are the branching functions that are defined by the decomposition (4.41). For the case at hand, the branching functions can be computed explicitly 39,

$$
\begin{aligned}
\psi_{\left[j_{1}, j_{2}, j_{3}\right]}^{\mathrm{WZW}}(q)= & \frac{1}{\eta(q)^{4}} \sum_{n, m=0}^{\infty}(-1)^{n+m} q^{\frac{m}{2}\left(m+4 j_{1}+2 n+1\right)+j_{1}+\frac{n}{2}-\frac{1}{8}} \\
& \times\left[q^{\left(j_{2}-\frac{n}{2}\right)^{2}}-q^{\left(j_{2}+\frac{n}{2}+1\right)^{2}}\right]\left[q^{\left(j_{3}-\frac{n}{2}\right)^{2}}-q^{\left(j_{3}+\frac{n}{2}+1\right)^{2}}\right] .
\end{aligned}
$$


geometric
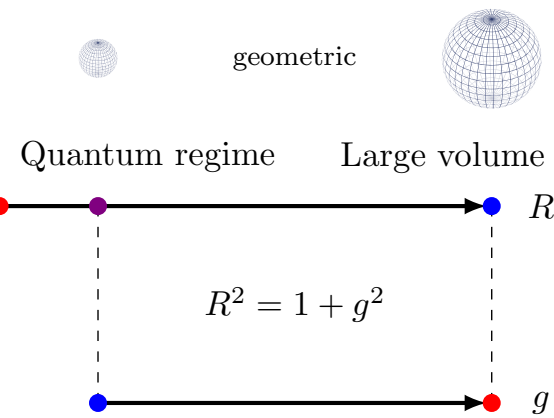

Weak coupling Strong coupling

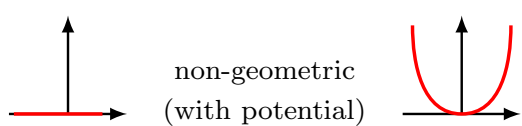

$\mathrm{S}^{3 \mid 2}$ Supersphere $\sigma$-model

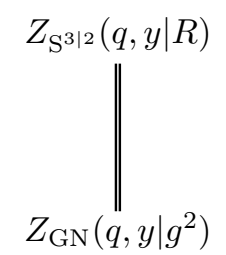

OSP(4|2) Gross-Neveu model

Figure 5: (Color online) The duality between the OSP(4|2) Gross-Neveu model and the $\mathrm{S}^{3 \mid 2}$ supersphere $\sigma$-model in pictures.

Using eq. (3.61), we are now prepared to construct the partition function at finite coupling $g^{2}$ :

$$
Z_{\mathrm{GN}}\left(q, y \mid g^{2}\right)=\sum_{\Lambda} q^{-\frac{1}{2} \frac{g^{2}}{1+g^{2}} C_{\Lambda}} \psi_{\Lambda}^{\mathrm{WZW}}(q) \chi_{\Lambda}(y)
$$

The validity of this formula has been checked against numerical results from lattice discretizations with impressive agreement [100].

Let us discuss the consequences of formula (4.43) in more detail, see also Figure 4. At zero coupling, the spectrum is characterized by the following features: All states have either integer or half-integer energy and at each energy level there is only a finite number of states. As mentioned above, these states are accounted for by the two affine $\widehat{\mathrm{OSP}}(4 \mid 2)_{1}$ representations built on top of the vacuum (with $h=0$ ) and the fundamental representation (with $h=1 / 2$ ), respectively. Once the deformation is switched on, the affine symmetry is broken and the states will receive an anomalous dimension depending on their transformation behavior under global $\operatorname{OSP}(4 \mid 2)$ transformations (the zero-modes of the current algebra). In particular, multiplets belonging to a representation with vanishing Casimir do not receive any correction. These are all protected BPS representations 22 This applies in particular to the adjoint representation and ensures that the currents stay at conformal dimension $h=1$.

At intermediate coupling the spectrum is very complicated, exhibiting almost no sign of an underlying organizing principle, except for the preserved global $\operatorname{OSP}(4 \mid 2)$ and the Virasoro symmetry. However, at infinite coupling we again recover a special situation. The energy of a multiplet $\Lambda$ is shifted by $-C_{\Lambda} / 2$ in this case. It can be shown that despite this shift all conformal dimensions remain non-negative. Even more surprising, the spectrum is very regular again, exhibiting an integer level spacing (as opposed to the half-integer spacing at $g=0$ ). Nevertheless the spectrum now has entirely different characteristics than at zero coupling. Indeed, at infinite coupling we find an infinite number of states on each energy level, see again Figure 4.

\subsubsection{Identification with supersphere sigma model}

We now wish to argue that the spectrum of the Gross-Neveu model discussed in the previous section coincides with the large volume partition function of the $\sigma$-model on the supersphere $\mathrm{S}^{3 \mid 2}$ when we send the coupling $g$ to infinity. At infinite volume the partition function is easy to write down since the fields $\vec{X}$ become free. The most general field is obtained by considering the normal ordered products $\prod \partial^{n_{i}} \bar{\partial}^{m_{i}} X^{a_{i}}$ of the fields $X^{a}$ and their derivatives and the energy (scaling dimension) of such a field is just given by the number of derivatives.

\footnotetext{
${ }^{22}$ It should be noted, however, that there are short/BPS representations for $\operatorname{OSP}(4 \mid 2)$ which are not protected in this sense.
} 
We assume Neumann boundary conditions, i.e. a freely moving open string. In this case we are only left with one type of derivative. In close analogy to the harmonic analysis on $\mathrm{S}^{3 \mid 2}$ (see Section 2.4.3) we can write down the open string partition function

$$
\begin{aligned}
Z_{\mathrm{S}^{3 \mid 2}}(q, y \mid R=\infty) & \\
= & \lim _{t \rightarrow 1} \prod_{n=1}^{\infty} \frac{\left(1-t^{2} q^{n}\right)\left(1+t q^{n} y_{3}\right)\left(1+t q^{n} / y_{3}\right)}{\left(1-t q^{n} y_{1} y_{2}\right)\left(1-t q^{n} y_{1} / y_{2}\right)\left(1-t q^{n} y_{2} / y_{1}\right)\left(1-t q^{n} / y_{1} y_{2}\right)}
\end{aligned}
$$

The only difference compared to eq. (2.41) are the additional terms involving powers $q^{n}$. These correspond to counting derivatives $\partial^{n} \vec{X}$ instead of plain coordinates $\vec{X}$. Since the constraint $\vec{X}^{2}=R^{2}$ also leads to constraints on derivatives of $\vec{X}$, also the first term in the numerator had to be extended to an infinite product.

Even though it is by no means obvious, the decomposition of the partition function (4.44) into irreducible characters of $\operatorname{OSP}(4 \mid 2)$ precisely agrees with the limit $g \rightarrow \infty$ of the expression (4.43) 39. This suggests that the moduli spaces of the two theories indeed overlap - and employing their common symmetry - actually coincide, see Figure 5 for an illustration. Complementary calculations based on either lattice models [100], background field methods or a cohomological reduction 81 confirm this picture and predict that the couplings should in fact be related as $R^{2}=1+g^{2}$.

\section{Conclusions and Outlook}

Our goal in this review was to present conformal field theories with internal supersymmetries as a vast and intriguing branch of logarithmic conformal field theory. We have seen a large family of models, including WZW models on supergroups and $\sigma$-models on coset superspaces, along with their deformations and some dualities. Many of these models possess profound applications to problems in different areas of theoretical physics.

To make our presentation reasonably self-contained we included some basic material, in particular on superalgebras and their representation theory. This also meant that we had to skip over quite a few further developments. These include a systematic discussion of boundary conditions. For WZW models on type I supergroups, these are quite will understood. At the example of the GL(1|1) WZW model it has been shown that Cardy's construction basically carries over to the logarithmic setting [94. Besides there also exist indications that the geometric characterization of D-branes in group manifolds extends from the purely bosonic case [113, 94, 135].

Another topic of considerable interest are GKO-type coset models for supergroups with and without world-sheet supersymmetry. Some first studies can be found in [136 and 137. It turns out that supergroup GKO coset models include several families of models which possess exactly marginal integrable deformations, generalizing the current-current deformations of WZW models we sketched in Section [3.5. These models along with their deformations and potential dualities certainly deserve further study.

On the more mathematical side, the relation of the representation theory of affine Lie superalgebras with Mock modular forms warrants more exploration, see [15] and [92, 93, 138, for some existing results. In our opinion, however, the most pressing questions concern the study of dualities between WZW and $\sigma$-models. The only example that is reasonably well understood right now is the duality between the $\operatorname{OSP}(4 \mid 2)$ WZW model and the $\sigma$-model on the supersphere $S^{3 \mid 2}$, see Section 4.3. It is likely that further progress requires a better understanding of the interplay between conformal invariance and integrability.

\section{Acknowledgments}

Much of the material presented in this review is based on joint work with a number of different collaborators. We would like to thank Constantin Candu, Thomas Creutzig, Gerhard Götz, Vladimir Mitev, Peter Rønne and Hubert Saleur for many discussions and pleasant collaboration. Besides we are indebted to the anonymous referees for providing extremely detailed feedback which helped 
improve our presentation. Thomas Quella is funded by the German Research Council (DFG) through M. Zirnbauer's Leibniz Prize, DFG grant no. ZI 513/2-1. Additional support is received from the DFG through the SFB|TR 12 "Symmetries and Universality in Mesoscopic Systems" and the Center of Excellence "Quantum Matter and Materials". The research of Volker Schomerus is supported in part by the SFB 676 "Particles, Strings, and the Early Universe" and the Marie Curie network GATIS (gatis.desy.eu) of the European Union's Seventh Framework Programme FP7/2007-2013/ under REA Grant Agreement No 317089.

\section{References}

[1] O. Aharony, S. S. Gubser, J. M. Maldacena, H. Ooguri, and Y. Oz, "Large N field theories, string theory and gravity," Phys. Rept. 323 (2000) 183-386, hep-th/9905111.

[2] G. Parisi and N. Sourlas, "Random magnetic fields, supersymmetry and negative dimensions," Phys. Rev. Lett. 43 (1979) 744.

[3] K. B. Efetov, "Supersymmetry and theory of disordered metals," Adv. Phys. 32 (1983) $53-127$.

[4] D. Bernard, "(Perturbed) conformal field theory applied to 2D disordered systems: An introduction," in Low-dimensional applications of quantum field theory, pp. 19-61. 1995. hep-th/9509137. Cargese Lectures in Theoretical Physics.

[5] G. Parisi and N. Sourlas, "Supersymmetric field theories and stochastic differential equations," Nucl. Phys. B206 (1982) 321.

[6] N. Read and H. Saleur, "Exact spectra of conformal supersymmetric nonlinear sigma models in two dimensions," Nucl. Phys. B613 (2001) 409, hep-th/0106124

[7] I. A. Gruzberg, A. W. W. Ludwig, and N. Read, "Exact exponents for the spin quantum Hall transition," Phys. Rev. Lett. 82 (1999) 4524, cond-mat/9902063.

[8] F. H. L. Essler, H. Frahm, and H. Saleur, "Continuum limit of the integrable $\operatorname{sl}(2 \mid 1) 3-\overline{3}$ superspin chain," Nucl. Phys. Br12 (2005) 513-572, cond-mat/0501197.

[9] H. Weidenmüller, "Single electron in a random potential and a strong magnetic field," Nucl. Phys. B290 (1987) 87-110.

[10] M. R. Zirnbauer, "Conformal field theory of the integer quantum Hall plateau transition," hep-th/9905054.

[11] M. J. Bhaseen, I. I. Kogan, O. A. Solovev, N. Tanigichi, and A. M. Tsvelik, "Towards a field theory of the plateau transitions in the integer quantum Hall effect," Nucl. Phys. B580 (2000) 688-720, cond-mat/9912060

[12] A. M. Tsvelik, "Evidence for the $P S L(2 \mid 2)$ Wess-Zumino-Novikov-Witten model as a model for the plateau transition in quantum Hall effect: Evaluation of numerical simulations," Phys. Rev. B75 (2007) 184201, cond-mat/0702611.

[13] J. Chalker and P. Coddington, "Percolation, quantum tunnelling and the integer Hall effect," J. Phys. C21 (1988) 2665-2679.

[14] M. R. Gaberdiel and H. G. Kausch, "A local logarithmic conformal field theory," Nucl. Phys. B538 (1999) 631-658, hep-th/9807091.

[15] A. M. Semikhatov, A. Taormina, and I. Y. Tipunin, "Higher level Appell functions, modular transformations, and characters," Comm. Math. Phys. 255 (2005) 469-512, math.qa/0311314

[16] J. Fuchs, S. Hwang, A. M. Semikhatov, and I. Y. Tipunin, "Nonsemisimple fusion algebras and the Verlinde formula," Commun. Math. Phys. 247 (2004) 713-742, hep-th/0306274 
[17] M. R. Gaberdiel and I. Runkel, "The logarithmic triplet theory with boundary," J. Phys. A39 (2006) 14745-14780, hep-th/0608184.

[18] R. R. Metsaev and A. A. Tseytlin, "Type IIB superstring action in $A d S_{5} \times S^{5}$ background," Nucl. Phys. B533 (1998) 109-126, hep-th/9805028.

[19] N. Berkovits, M. Bershadsky, T. Hauer, S. Zhukov, and B. Zwiebach, "Superstring theory on $A d S_{2} \times S^{2}$ as a coset supermanifold," Nucl. Phys. B567 (2000) 61-86, hep-th/9907200.

[20] D. Kagan and C. A. S. Young, "Conformal sigma-models on supercoset targets," Nucl. Phys. B745 (2006) 109-122, hep-th/0512250.

[21] A. Babichenko, "Conformal invariance and quantum integrability of sigma models on symmetric superspaces," Phys. Lett. B648 (2007) 254-261, hep-th/0611214.

[22] N. Berkovits, C. Vafa, and E. Witten, "Conformal field theory of AdS background with Ramond-Ramond flux," JHEP 03 (1999) 018, hep-th/9902098.

[23] M. Bershadsky, S. Zhukov, and A. Vaintrob, " $P S L(n \mid n)$ sigma model as a conformal field theory," Nucl. Phys. B559 (1999) 205-234, hep-th/9902180

[24] G. Götz, T. Quella, and V. Schomerus, "The WZNW model on PSU (1,1|2)," JHEP 03 (2007) 003, hep-th/0610070

[25] I. Bena, J. Polchinski, and R. Roiban, "Hidden symmetries of the $A d S_{5} \times S^{5}$ superstring," Phys. Rev. D69 (2004) 046002, hep-th/0305116.

[26] C. A. S. Young, "Non-local charges, $Z_{m}$ gradings and coset space actions," Phys. Lett. B632 (2006) 559-565, hep-th/0503008

[27] V. Gurarie, "Logarithmic operators in conformal field theory," Nucl. Phys. B410 (1993) 535-549, hep-th/9303160.

[28] H. G. Kausch, "Symplectic fermions," Nucl. Phys. B583 (2000) 513-541, hep-th/0003029.

[29] M. R. Gaberdiel, "An algebraic approach to logarithmic conformal field theory," Int. J. Mod. Phys. A18 (2003) 4593-4638, hep-th/0111260.

[30] M. Flohr, "Bits and pieces in logarithmic conformal field theory," Int. J. Mod. Phys. A18 (2003) 4497-4592, hep-th/0111228.

[31] L. Rozansky and H. Saleur, "Quantum field theory for the multivariable Alexander-Conway polynomial," Nucl. Phys. B376 (1992) 461-509.

[32] L. Rozansky and H. Saleur, "S and T matrices for the $U(1 \mid 1)$ WZW model: Application to surgery and three manifolds invariants based on the Alexander-Conway polynomial," Nucl. Phys. B389 (1993) 365-423, hep-th/9203069.

[33] Z. Maassarani and D. Serban, "Non-unitary conformal field theory and logarithmic operators for disordered systems," Nucl. Phys. B489 (1997) 603-625, hep-th/9605062.

[34] S. Guruswamy, A. Le Clair, and A. W. W. Ludwig, " $g l(N \mid N)$ super-current algebras for disordered Dirac fermions in two dimensions," Nucl. Phys. B583 (2000) 475-512, cond-mat/9909143

[35] A. W. W. Ludwig, "A free field representation of the $\operatorname{ssp}(2 \mid 2)$ current algebra at level $k=-2$, and Dirac fermions in a random $S U(2)$ gauge potential," cond-mat/0012189.

[36] V. Schomerus and H. Saleur, "The GL(1|1) WZW model: From supergeometry to logarithmic CFT," Nucl. Phys. B734 (2006) 221-245, hep-th/0510032

[37] H. Saleur and V. Schomerus, "On the $S U(2 \mid 1)$ WZW model and its statistical mechanics applications," Nucl. Phys. B775 (2007) 312-340, hep-th/0611147. 
[38] T. Quella and V. Schomerus, "Free fermion resolution of supergroup WZNW models," JHEP 09 (2007) 085, arXiv:0706.0744.

[39] V. Mitev, T. Quella, and V. Schomerus, "Principal chiral model on superspheres," JHEP 11 (2008) 086, arXiv:0809.1046.

[40] V. G. Kac, "Lie superalgebras," Adv. Math. 26 (1977) 8-96.

[41] M. Scheunert, W. Nahm, and V. Rittenberg, "Classification of all simple graded Lie algebras whose Lie algebra is reductive. 1," J. Math. Phys. 17 (1976) 1626.

[42] P. Di Francesco, P. Mathieu, and D. Senechal, Conformal Field Theory. Graduate Texts in Contemporary Physics. Springer, New York, 1999.

[43] J. Fuchs, Affine Lie algebras and quantum groups. Cambridge Monographs on Mathematical Physics. Cambridge University Press, Cambridge, 1995.

[44] J. Fuchs and C. Schweigert, Symmetries, Lie algebras and representations. Cambridge Monographs on Mathematical Physics. Cambridge University Press, Cambridge, 1997.

[45] L. Frappat, P. Sorba, and A. Sciarrino, Dictionary on Lie algebras and superalgebras. Academic Press Inc., San Diego, CA, 2000. hep-th/9607161. Extended and corrected version of the E-print [hep-th/9607161].

[46] V. G. Kac and M. Wakimoto, "Integrable highest weight modules over affine superalgebras and number theory," in Lie theory and geometry, vol. 123 of Progr. Math., pp. 415-456. Birkhäuser Boston, Boston, MA, 1994. hep-th/9407057.

[47] W. Nahm, "Supersymmetries and their representations," Nucl. Phys. B135 (1978) 149.

[48] N. Berkovits, "Simplifying and extending the $A d S_{5} \times S^{5}$ pure spinor formalism," JHEP 09 (2009) 051, arXiv:0812.5074.

[49] V. G. Kac, "Characters of typical representations of classical Lie superalgebras," Comm. Algebra 5 (1977) 889-897.

[50] V. Kac, "Representations of classical Lie superalgebras," in Differential geometrical methods in mathematical physics, II (Proc. Conf., Univ. Bonn, Bonn, 1977), vol. 676 of Lecture Notes in Math., pp. 597-626. Springer, Berlin, 1978.

[51] S.-J. Cheng and W. Wang, Dualities and representations of Lie superalgebras, vol. 144 of Graduate Studies in Mathematics. American Mathematical Society, Providence, RI, 2012.

[52] J. Germoni, "Indecomposable representations of special linear Lie superalgebras," J. Algebra 209 (1998) 367-401.

[53] V. Serganova, "Characters of irreducible representations of simple Lie superalgebras," in Proceedings of the International Congress of Mathematicians, Vol. II (Berlin, 1998), no. Extra Vol. II, pp. 583-593 (electronic). 1998.

[54] J. Brundan, "Kazhdan-Lusztig polynomials and character formulae for the Lie superalgebra gl $(m \mid n), " J$. Amer. Math. Soc. 16 (2003) 185-231 (electronic), math.RT/0203011.

[55] J. Brundan and C. Stroppel, "Highest weight categories arising from Khovanov's diagram algebra IV: the general linear supergroup," J. Eur. Math. Soc. (JEMS) 14 (2012) 373-419 arXiv:0907.2543.

[56] C. Gruson and V. Serganova, "Cohomology of generalized supergrassmannians and character formulae for basic classical Lie superalgebras," Proc. Lond. Math. Soc. 101 (2010) 852-892, arXiv:0906.0918,

[57] I. M. Musson and V. Serganova, "Combinatorics of character formulas for the Lie superalgebra $g l(m, n), "$ Transform. Groups 16 (2011) 555-578, arXiv:1104.1668. 
[58] S.-J. Cheng, N. Lam, and W. Wang, "Super duality and irreducible characters of ortho-symplectic Lie superalgebras," Invent. Math. 183 (2011) 189-224, arXiv:0911.0129

[59] J. Germoni, "Indecomposable representations of $\operatorname{osp}(3 \mid 2), D(2,1 ; \alpha)$ and $G(3)$," Bol. Acad. Nac. Cienc. (Córdoba) 65 (2000) 147-163. Colloquium on Homology and Representation Theory (Spanish) (Vaquerí as, 1998).

[60] G. Götz, T. Quella, and V. Schomerus, "Representation theory of $\operatorname{sl}(2 \mid 1), "$ J. Algebra 312 (2007) 829-848, hep-th/0504234.

[61] G. Götz, T. Quella, and V. Schomerus, "Tensor products of $p s l(2 \mid 2)$ representations," hep-th/0506072.

[62] F. W. Anderson and K. R. Fuller, Rings and categories of modules. Springer, 1992.

[63] J. Brundan, "Tilting modules for Lie superalgebras," Comm. Algebra 32 (2004) 2251-2268, math.RT/0209235

[64] J. E. Humphreys, Representations of semisimple Lie algebras in the $B G G$ category $O$, vol. 94 of Graduate Studies in Mathematics. American Mathematical Society, Providence, RI, 2008.

[65] I. M. Musson, Lie superalgebras and enveloping algebras, vol. 131 of Graduate Studies in Mathematics. American Mathematical Society, Providence, RI, 2012.

[66] Y. M. Zou, "Categories of finite-dimensional weight modules over type I classical Lie superalgebras," J. Algebra 180 (1996) 459-482.

[67] V. Serganova, "Blocks in the category of finite-dimensional representations of $g l(m \mid n)$." Preprint available on V. Serganova's homepage, 2006.

[68] V. Mitev, T. Quella, and V. Schomerus, "Conformal superspace sigma-models," J. Geom. Phys. 61 (2011) 1703-1716, arXiv:1210.8159.

[69] E. Witten, "Notes on supermanifolds and integration," arXiv:1209.2199.

[70] D. A. Leites, "Introduction to the theory of supermanifolds," Russian Mathematical Surveys 35 (1980) 1-64

[71] V. S. Varadarajan, Supersymmetry for mathematicians: an introduction, vol. 11 of Courant Lecture Notes in Mathematics. New York University Courant Institute of Mathematical Sciences, New York, 2004.

[72] C. Carmeli, L. Caston, and R. Fioresi, Mathematical foundations of supersymmetry. EMS Series of Lectures in Mathematics. European Mathematical Society (EMS), Zürich, 2011.

[73] A. Alldridge, J. Hilgert, and T. Wurzbacher, Calculus on supermanifolds. Book in preparation.

[74] Y. I. Manin, Gauge Field Theory and Complex Geometry. Grundlehren der mathematischen Wissenschaften. Springer, 1998.

[75] P. Deligne and J. W. Morgan, "Notes on supersymmetry (following Joseph Bernstein)," in Quantum fields and strings: a course for mathematicians, Vol. 1, 2 (Princeton, NJ, 1996/1997), pp. 41-97. Amer. Math. Soc., Providence, RI, 1999.

[76] C. Bartocci, U. Bruzzo, and D. Hernández Ruipérez, The geometry of supermanifolds vol. 71 of Mathematics and its Applications. Kluwer Academic Publishers Group, Dordrecht, 1991.

[77] B. DeWitt, Supermanifolds. Cambridge Monographs on Mathematical Physics. Cambridge University Press, Cambridge, 1992. 
[78] G. M. Tuynman, Supermanifolds and supergroups - Basic theory, vol. 570 of Mathematics and its Applications. Kluwer Academic Publishers, Dordrecht, 2004.

[79] A. Rogers, Supermanifolds - Theory and applications. World Scientific Publishing, 2007.

[80] K. Coulembier, "The orthosymplectic supergroup in harmonic analysis," J. Lie Theory 23 (2013) 55-83, arXiv:1202.0668

[81] C. Candu, T. Creutzig, V. Mitev, and V. Schomerus, "Cohomological reduction of sigma models," JHEP 05 (2010) 047, arXiv:1001.1344

[82] C. Candu, V. Mitev, T. Quella, H. Saleur, and V. Schomerus, "The sigma model on complex projective superspaces," JHEP 02 (2010) 015, arXiv:0908.0878.

[83] E. Witten, "Nonabelian bosonization in two dimensions," Commun. Math. Phys. 92 (1984) 455-472.

[84] S. J. Gates, M. T. Grisaru, M. Rocek, and W. Siegel, "Superspace, or one thousand and one lessons in supersymmetry," Front. Phys. 58 (1983) 1-548, hep-th/0108200

[85] A. M. Polyakov and P. B. Wiegmann, "Goldstone fields in two-dimensions with multivalued actions," Phys. Lett. B141 (1984) 223-228.

[86] V. G. Kac and M. Wakimoto, "Integrable highest weights modules over affine superalgebras and Appell's function," Comm. Math. Phys. 215 (2001) 631-682, math-ph/0006007.

[87] V. Serganova, "Kac-Moody superalgebras and integrability," in Developments and trends in infinite-dimensional Lie theory, vol. 288 of Progr. Math., pp. 169-218. Birkhäuser Boston Inc., Boston, MA, 2011.

[88] M. Gorelik, "Weyl denominator identity for affine Lie superalgebras with non-zero dual Coxeter number," J. Algebra 337 (2011) 50-62, arXiv:0911.5594.

[89] M. Gorelik and S. Reif, "A denominator identity for affine Lie superalgebras with zero dual Coxeter number," Algebra Number Theory 6 (2012) 1043-1059, arXiv:1012.5879.

[90] V. Serganova, "Structure and representation theory of Kac-Moody superalgebras,"] in Highlights in Lie algebraic methods, vol. 295 of Progr. Math., pp. 65-102. Birkhäuser/Springer, New York, 2012.

[91] S. Zwegers, Mock theta functions. PhD thesis, Utrecht University, 2002. arXiv:0807.4834.

[92] A. Folsom, "Kac-Wakimoto characters and universal mock theta functions," Trans. Amer. Math. Soc. 363 (2011) 439-455

[93] K. Bringmann and K. Ono, "Some characters of Kac and Wakimoto and nonholomorphic modular functions," Math. Ann. 345 (2009) 547-558.

[94] T. Creutzig, T. Quella, and V. Schomerus, "Branes in the GL(1|1) WZNW model," Nucl. Phys. B792 (2008) 257-283, arXiv:0708.0583,

[95] J. M. Maldacena and H. Ooguri, "Strings in $A d S_{3}$ and the $S L(2, R)$ WZW model. I," J. Math. Phys. 42 (2001) 2929-2960, hep-th/0001053.

[96] D. Gepner and E. Witten, "String theory on group manifolds," Nucl. Phys. B278 (1986) 493.

[97] B. L. Feigin and E. V. Frenkel, "Representations of affine Kac-Moody algebras, bosonization and resolutions," Lett. Math. Phys. 19 (1990) 307-317.

[98] J. Rasmussen, "Free field realizations of affine current superalgebras, screening currents and primary fields," Nucl. Phys. B510 (1998) 688-720, hep-th/9706091. 
[99] C. Candu and H. Saleur, "A lattice approach to the conformal $O S p(2 S+2 \mid 2 S)$ supercoset sigma model. Part I: Algebraic structures in the spin chain. The Brauer algebra," Nucl. Phys. B808 (2009) 441-486, arXiv:0801.0430,

[100] C. Candu and H. Saleur, "A lattice approach to the conformal $O S p(2 S+2 \mid 2 S)$ supercoset sigma model. Part II: The boundary spectrum," Nucl. Phys. B808 (2009) 487-524, arXiv:0801.0444

[101] C. Candu, Discrétisation des modèles sigma invariants conformes sur des supersphères et superespaces projectifs. PhD thesis, University Pierre and Marie Curie - Paris 6, 2008.

[102] F. Lesage, P. Mathieu, J. Rasmussen, and H. Saleur, "The $\widehat{s u}(2)_{-1 / 2}$ WZW model and the B $\gamma$ system," Nucl. Phys. B647 (2002) 363-403, hep-th/0207201.

[103] D. Ridout, "Fusion in fractional level $\widehat{s l}(2)$-theories with $k=-1 / 2, "$ Nucl. Phys. B848 (2011) 216-250, arXiv:1012.2905.

[104] A. N. Schellekens and S. Yankielowicz, "Simple currents, modular invariants and fixed points," Int. J. Mod. Phys. A5 (1990) 2903-2952.

[105] F. Lesage, P. Mathieu, J. Rasmussen, and H. Saleur, "Logarithmic lift of the $\widehat{s u}(2)_{-1 / 2}$ model," Nucl. Phys. B686 (2004) 313, hep-th/0311039.

[106] D. Ridout, "sl(2) $-1 / 2$ : A case study," Nucl. Phys. B814 (2009) 485-521, arXiv:0810.3532.

[107] D. Ridout, "sl(2) $-1 / 2$ and the triplet model," Nucl. Phys. B835 (2010) 314-342, arXiv:1001.3960,

[108] T. Creutzig and D. Ridout, "Modular data and Verlinde formulae for fractional level WZW models I," Nucl. Phys. B865 (2012) 83-114, arXiv:1205.6513.

[109] S. K. Ashok, R. Benichou, and J. Troost, "Conformal current algebra in two dimensions," JHEP 06 (2009) 017, arXiv:0903.4277.

[110] S. K. Ashok, R. Benichou, and J. Troost, "Asymptotic symmetries of string theory on $A d S_{3} \times S^{3}$ with Ramond-Ramond fluxes," JHEP 10 (2009) 051, arXiv:0907.1242

[111] R. Benichou and J. Troost, "The conformal current algebra on supergroups with applications to the spectrum and integrability," JHEP 04 (2010) 121, arXiv:1002.3712,

[112] A. Konechny and T. Quella, "Non-chiral current algebras for deformed supergroup WZW models," JHEP 1103 (2011) 124, arXiv:1011.4813.

[113] T. Quella, V. Schomerus, and T. Creutzig, "Boundary spectra in superspace sigma models," JHEP 10 (2008) 024, arXiv:0712.3549.

[114] H. Obuse, A. R. Subramaniam, A. Furusaki, I. A. Gruzberg, and A. W. W. Ludwig, "Boundary multifractality at the integer quantum Hall plateau transition: Implications for the critical theory," Phys. Rev. Lett. 101 (2008) 116802, arXiv:0804.2409.

[115] C. Candu, V. Mitev, and V. Schomerus, "Anomalous dimensions in deformed WZW models on supergroups," JHEP 1303 (2013) 003 , arXiv:1211.2238,

[116] D. Gepner, "Space-time supersymmetry in compactified string theory and superconformal models," Nucl. Phys. B296 (1988) 757.

[117] A. Giveon and M. Rocek, "On the BRST operator structure of the N=2 string," Nucl. Phys. B400 (1993) 145-160, arXiv:hep-th/9302049.

[118] N. Berkovits, "Covariant quantization of the Green-Schwarz superstring in a Calabi-Yau background," Nucl. Phys. B431 (1994) 258-272, arXiv:hep-th/9404162.

[119] N. Berkovits, "Quantization of the type II superstring in a curved six- dimensional background," Nucl. Phys. B565 (2000) 333-344, hep-th/9908041. 
[120] A. Cagnazzo and K. Zarembo, "B-field in $A d S_{3} / C F T_{2}$ correspondence and integrability," JHEP 1211 (2012) 133, arXiv:1209.4049

[121] M. R. Gaberdiel and S. Gerigk, "The massless string spectrum on $A d S_{3} \times S^{3}$ from the supergroup," JHEP 1110 (2011) 045, arXiv:1107.2660.

[122] S. Gerigk, "String states on $A d S_{3} \times S^{3}$ from the supergroup," JHEP 1210 (2012) 084 arXiv: 1208.0345 .

[123] J. Troost, "Massless particles on supergroups and $A d S_{3} \times S^{3}$ supergravity," JHEP 07 (2011) 042, arXiv:1102.0153.

[124] N. Berkovits, "Super-Poincaré covariant quantization of the superstring," JHEP 04 (2000) 018, hep-th/0001035.

[125] N. Berkovits, "Quantum consistency of the superstring in $A d S_{5} \times S^{5}$ background," JHEP 0503 (2005) 041, arXiv:hep-th/0411170

[126] B. C. Vallilo, "Flat currents in the classical $A d S_{5} \times S^{5}$ pure spinor superstring," JHEP 0403 (2004) 037, arXiv:hep-th/0307018.

[127] R. Benichou, "First-principles derivation of the AdS/CFT Y-systems," JHEP 1110 (2011) 112, arXiv:1108.4927

[128] P. Fre and P. A. Grassi, "Pure spinor formalism for $O s p(N \mid 4)$ backgrounds," Int. J. Mod. Phys. A27 (2012) 1250185, arXiv:0807.0044

[129] D. Sorokin, A. Tseytlin, L. Wulff, and K. Zarembo, "Superstrings in $A d S_{2} \times S^{2} \times T^{6}$," J. Phys. A44 (2011) 275401, arXiv:1104.1793.

[130] J. Rahmfeld and A. Rajaraman, "The GS string action on $A d S_{3} \times S^{3}$ with Ramond-Ramond charge," Phys. Rev. D60 (1999) 064014, hep-th/9809164.

[131] G. Arutyunov and S. Frolov, "Superstrings on $A d S_{4} \times C P^{3}$ as a coset sigma-model," JHEP 09 (2008) 129, arXiv:0806.4940.

[132] K. Zarembo, "Strings on semisymmetric superspaces," JHEP 05 (2010) 002, arXiv:1003.0465,

[133] A. Cagnazzo, D. Sorokin, A. A. Tseytlin, and L. Wulff, "Semiclassical equivalence of Green-Schwarz and pure-spinor/hybrid formulations of superstrings in $A d S_{5} \times S^{5}$ and $A d S_{2} \times S^{2} \times T^{6}, "$ J. Phys. A46 (2013) 065401, arXiv:1211.1554

[134] E. Witten, "Phases of $N=2$ theories in two-dimensions," Nucl. Phys. B403 (1993) 159-222, arXiv:hep-th/9301042.

[135] T. Creutzig, "Geometry of branes on supergroups," Nucl. Phys. B812 (2009) 301-321, arXiv:0809.0468

[136] T. Creutzig, P. B. Rønne, and V. Schomerus, "N=2 superconformal symmetry in super coset models," Phys. Rev. D80 (2009) 066010, arXiv:0907.3902,

[137] C. Candu and V. Schomerus, "Exactly marginal parafermions," Phys. Rev. D84 (2011) 051704, arXiv:1104.5028,

[138] C. Alfes and T. Creutzig, "The Mock modular data of a family of superalgebras," arXiv:1205.1518 